\title{
Radiation Embrittlement of the Neutron Shield Tank from the Shippingport Reactor
}

Manuscript Completed: July 1991

Date Published: October 1991

\section{DISCLAIMER}

Prepared by

O. K. Chopra, W. J. Shack, S. T. Rosinski*

Argonne National Laboratory

9700 South Cass Avenue

Argonne, IL 60439

Prepared for

Division of Engineering

Office of Nuclear Regulatory Research

U.S. Nuclear Regulatory Commission

Washington, DC 20555

NRC FIN A2256

* Sandia National Laboratories

Albuquerque, NM 87185
This report was prepared as an account of work sponsored by an agency of the United States This report was prepared as an account of work sponsored any agency thereof, nor any of their Government. Neither the United States Government nor any agency thal liability or responsiemployees, makes any warranty, express or implied, or assumes any legal liability, product, or bility for the accuracy, completeness, or usefulness or any information, apparatus, proder process disclosed, or represents that its use would not infringe privately owned rights. Reference herein to any specific commercial product, process, or service by trade name, trademark, manufacturer, or otherwise does not necessarily constitute or imply its endorsement, recommendation, or favoring by the United States Government or any agency thereof The of the and opinions of authors expressed hercin do not necessarily state or reflect those of the United States Government or any agency thereof. 


\title{
Radiation Embrittlement of the Neutron Shield Tank from the Shippingport Reactor
}

\author{
O. K. Chopra, W. J. Shack, and S. T. Rosinskd
}

\begin{abstract}
The irradiation embrittlement of neutron shield tank (NST) material (A212 Grade B steel) from the Shippingport reactor has been characterized. Irradiation increases the Charpy transition temperature (CTT) by $23-28^{\circ} \mathrm{C}\left(41-50^{\circ} \mathrm{F}\right)$ and decreases the upper-shelf energy. The shift in CTT is not as severe as that observed in high-flux isotope reactor (HFIR) survellance specimens. However, the actual value of the CTT is higher than that for the HFIR data. The increase in yield stress is $51 \mathrm{MPa}(7.4 \mathrm{ksi})$, which is comparable to HFIR data. The NST material is weaker in the transverse orientation than in the longitudinal orientation. Some effects of position across the thickness of the wall are also observed: the CTT shift is slightly greater for specimens from the inner region of the wall. Annealing studies indicate complete recovery from embrittlement after $1 \mathrm{~h}$ at $400^{\circ} \mathrm{C}\left(752^{\circ} \mathrm{F}\right)$. Although the weld metal is significantly tougher than the base metal, the shifts in CTT are comparable. The shifts in CTT for the Shippingport NST are consistent with the test and Army reactor data for irradiations at $<232^{\circ} \mathrm{C}\left(<450^{\circ} \mathrm{F}\right)$ and show very good agreement with the results for HFIR A212-B steel irradiated in the Oak Ridge Research Reactor (ORR). The effects of irradiation temperature, fluence rate, and neutron flux spectrum are discussed. The results indicate that fluence rate has no effect on radiation embrittlement at rates as low as $2 \times 10^{8} \mathrm{n} / \mathrm{cm}^{2} \cdot \mathrm{s}$ and at the low operating temperatures of the Shippingport NST, i.e. $55^{\circ} \mathrm{C}\left(130^{\circ} \mathrm{F}\right)$. This suggests that the accelerated embrittlement of HFIR survelllance samples is most likely due to the relatively higher proportion of thermal neutrons in the HFIR spectrum compared to that for the test reactors.
\end{abstract}




\section{Contents}

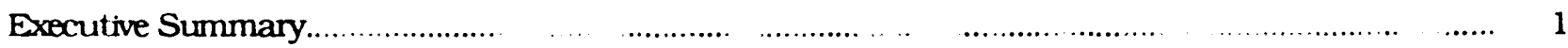

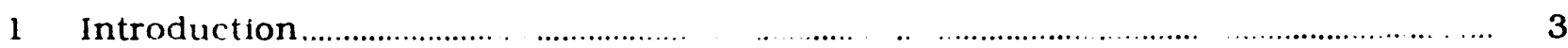

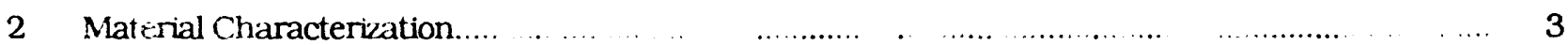

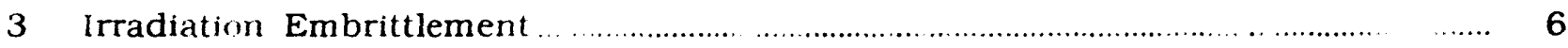

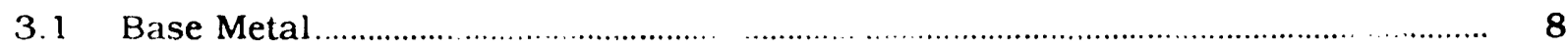

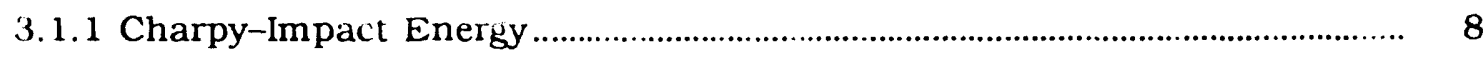

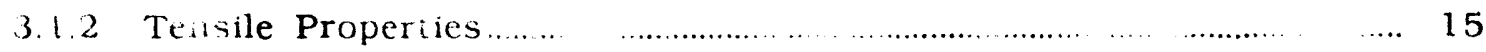

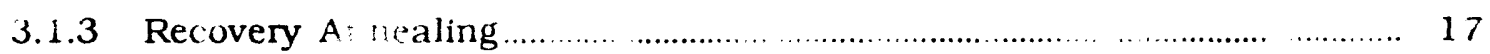

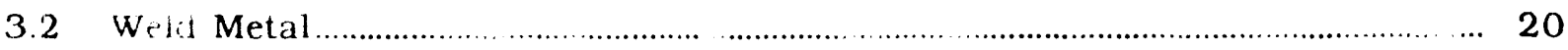

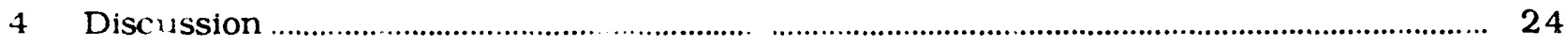

4.1 ATR Survellance I)

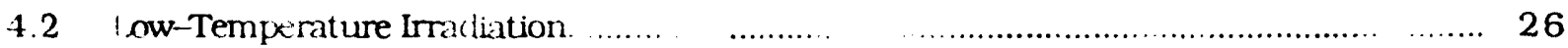

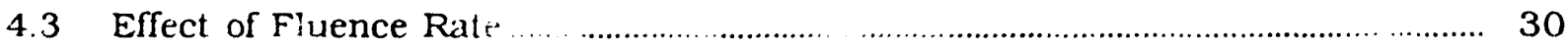

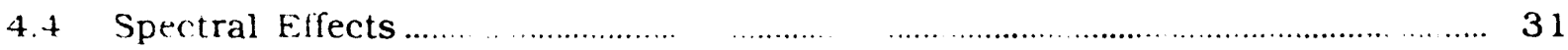

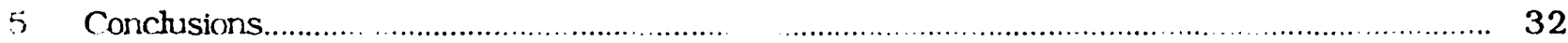

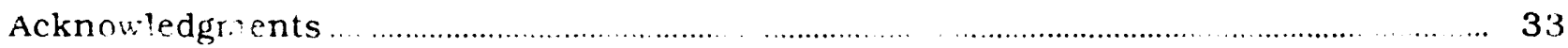

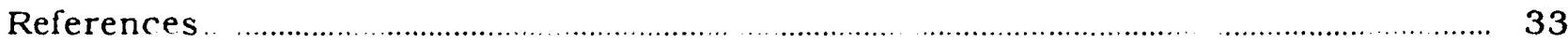

\section{List of Figures}

1. Schematic representation of the NST support for the Shippingport reactor

2. Layout of sample location in the Shippingport NST inner and outer walls ................ 4

3. Micrographs of the Shippingport NST !naterial "long transverse and rolling sections. 
4. Hardness profile across the thickness of the Shippingport NST outer wall

5. Hardness profiles for Locatio $:=3$ and 9 of the Shippingport NST inner wall ...............

6. Hardness profile for Location 8 of the Shippingport NST inner wall

7. Cutting diagram for base-metal and weld-metal specimens from irradiated and shielded walls of the Shippingport NST.

8. Best-fit Charpy transition curves for LT and TL specimens from the inner- and outer-10-mm regions of the shielded wall of the Shippingport NST.

9. Best-fit Charpy transition curves for LT and TL specimens from the center-10mm region of the shielded wall of the Shippingport NST

10. Best-fit Charpy transition curve for LT specimens from Location LA of the shielded wall of the Shippingport NST.

11. Best-fit Charpy transition curves for outer-region LT specimens, inner-region LT specimens, and TL specimens from all regions of the irradiated wall of the Shippingport NST.

12. Yield stress estimated from Charpy-impact tests and measured from tensile tests for irradiated and shielded walls of the Shippingport NST

13. Change in hardness of the shielded wall after annealing at $400^{\circ} \mathrm{C}$

14. Change in hardness measured on Sections A, B, C, and D of the irradiated wall after annealing at $400^{\circ} \mathrm{C}$.

15. Charpy transition curves for annealed TL specimens from the irradiated and the shielded wall of the Shippingport NST

16. Charpy transition curves for weld metal specimens from the inner- and outer10-mm regions of the shielded wall of the Shippingport NST.

17. Charpy transition curves for weld metal specimens from inner region and outer region of the irradiated wall of the Shippingport NST.

18. Comparison of Charpy transition curves for nonirradiated and irradiated HFIR survellance samples and material from the Shippingport NST

19. Shifts in CTT with neutron fluence for the Shippingport NST material, HFIR surveillance samples, and HFIR A212-B irradiated in the ORR.

20. Comparison of CTT shifts for the Shippingport NST and for samples irradiated in the ORR with data from test reactors. 
21. Comparison of CTT shifts for the Shippingport NST and ORR-irradiated specimens with test reactor data for irradiations at $<93^{\circ} \mathrm{C}$

22. Shifts in CTT with neutron fluence for ASTM reference A212-B and A302-B steels irradiated at $277-310^{\circ} \mathrm{C}$

23. Comparison of CTT shifts for HFIR surveillance samples, the Shippingport NST,

and ORR-irradiated sauiples with data from test and Army reactors.

24. Comparison of shifts in CTT with dpa for HFIR surveillance samples, the Shippingport NST, and ORR irradiation with data from test reactor.

25. Plots of dpa $(E>0.1 \mathrm{MeV})$ vs. dpa rate for specific values of CTT shifts

\section{List of Tables}

1. Typical Composit on (wt.\%) of plate and weld metal from the Shippingport NST.... 5

2. Charpy-impact data for the irradiated inner wall of the Shippingport NST.............. 9

3. Charpy-impact data for the shielded outer wall of the Shippingport NST................. 10

4. Charpy-impact data for Location LA of the shielded outer wall of the Shippingport NST.

5. Values of constants in Eq. 1, CTT, and USE for A212 Grade B material from shielded and irradiated walls of the Shippingport NST.

6. Tensile test results for irradiated and shielded walls of the Shippingport NST

7. Hardness values (Rockwell B) of the Shippingport NST material from irradiated and shielded walls after annealing at $400^{\circ} \mathrm{C}$.

8. Charpy-impact data for material from the irradiated and shielded walls of the Shippingport NST after annealing at $400^{\circ} \mathrm{C}$.

9. Charpy impact data for the shielded outer-wall weld of the Shippingport NST

10. Charpy impact data for the irradiated inner-wall weld of the Shippingport NST......

11. Values of constants in Eq. 1, CTT, and USE for weld metal from the shielded and irradiated walls of the Shippingport NST.

12. Summary of Charpy-impact and tensile results for HFIR surveillance tests, for samples irradiated in the ORR, and for the Shippingport NST. 
13. Chemical compositions (wt.\%) of territit steeis from the HFIR surveillance program 
Data on surveillance specimens from the high-nux isotope reactor (HFIR) at Oak Ridge National Laboratory showed a very high degree of embrittlement when compared with data obtained on similar materials in test reactors. The difference between the HFIR and test reactor data has been attributed to a fluence-rate effect, 1.e.. the degree of embrittlement per unit of fast fluence increases at low neutron flux, and/or to a softened neutron spectrum, i.e., a high thermal-to-fast-neutron-flux ratio may contribute to accelerated embrittlement. Current Nuclear Regulatory Commission guidelines for the assessment of embrittlement of pressure vessel support structures of commercial light-water reactors do not consider the contributions of fuence rate or spectral effects. HFIR results raise the possibility that the guidelines may not be sufficiently conservative.

To help resolve this issue, a program was initiated to characterize the irradiation embrittlement of the neutron shield tank (NST) from the decomimissioned Shippingport reactor. The Shippingport NST, which operated at $55^{\circ} \mathrm{C}\left(130^{\circ} \mathrm{F}\right)$, was fabricated from rolled A2 12 Grade B steel similar to that used for the HFIR vessel. The inner vrall of the NST was exposed to a total rnaximum fluence of $\approx 6 \times 10^{17} \mathrm{n} / \mathrm{cm}^{2}(\mathrm{E}>1 \mathrm{MeV})$ over a life of $9.25 \mathrm{ef}$ fective-full-power years (efpy). This corresponds to a fast flux of $\approx 2 \times 10^{9} \mathrm{n} / \mathrm{cm}^{2} \cdot \mathrm{s}$. The HFIR surveillance specimens were exposed at a temperature of $50^{\circ} \mathrm{C}\left(122^{\circ} \mathrm{F}\right)$ over a period of $\approx 17$ efpy; the flux of fast neutrons was $\approx 2 \times 10^{8} \mathrm{n} / \mathrm{cm}^{2} . \mathrm{s}$.

Eight disc samples, $\approx 155 \mathrm{~mm}$ in diameter, of the base metal and three weld samples were obtained from the inner wall of the NST, along with corresponding samples from the outer wall. Material characterization was carried out to determine chemical composition, grain structure, and hardness. Significant variations in hardness were observed across the thickness of the NST wall; the hardness values of the inner- and outer-7-mm regions of the plate were $\approx 10 \%$ higher than those of the plate center. Irradiation embrittlement was characterized by Charpy-impact and tensile tests, as well as by hardness measurements. Specimens were obtained in longitudinal (LT) and transverse (TL) orientations from three 10-mm-wide regions (inner, center, and outer) across the thickness of the NST wall. Samples from the outer wall, with about 6 orders of magnitude lower fluence, were used to determine baseline data for nonirradiated material.

Results indicate that the increase in Charpy transition temperature (CTT) for the Shippingport NST is not as severe as that observed for HFIR surveillance samples. The actual value of CTT, however, is higher than that for HFIR A212-B steel. The shifts in CTT at fluence levels between 3 and $6 \times 10^{17} \mathrm{n} / \mathrm{cm}^{2}(\mathrm{E}>1 \mathrm{MeV})$ are $23-28^{\circ} \mathrm{C}\left(41-50^{\circ} \mathrm{F}\right)$ for both LT and TL orientations. The TL orientation is weaker than the LT orientation, i.e., the CTT is higher and upper-shelf energy is lower. Increases in yield stress and hardness were $51 \mathrm{MPa}(7.4 \mathrm{ksi})$ and $12-18 \mathrm{DPH}$, respectively. Annealing studies indicate complete recovery from embrittlement after $1 \mathrm{~h}$ at $400^{\circ} \mathrm{C}\left(752^{\circ} \mathrm{F}\right)$. Although the weld metal is significantly tougher than the ba 2 metal, the shifts in CTT are comparable. The mechanical-property changes for the Shippingport NST agree well with the correlations between increases in CTT, yield stress, and hardness that have been developed for pressure vessel steels.

Radiation embrittlement of the Shippingport NST A212-B steel is consistent with the available data for irradiations at $<232^{\circ} \mathrm{C}\left(<450^{\circ} \mathrm{F}\right)$ and shows very good agreement with the 
data from test and Army reactors. The results indicate that at the low operating temperatures of the Shippingport NST, 1.e., $55^{\circ} \mathrm{C}\left(130^{\circ} \mathrm{F}\right)$ fluence rate has no effect on radiation embrittlement at rates as low as $2 \times 10^{8} \mathrm{n} / \mathrm{cm}^{2} \cdot \mathrm{s}$. The accelerated embrittlement of HFIR survellance samples is most likely due to the relatively higher proportion of thermal neutrons in the HFIR spectrum compared to that for the test reactors. 
Data on suiveillance specimens from the high-flux isotope reactor (HFIR) at Oak Ridge National Laboratory showed a very high degree of radiation-induced embrittlement in A2 12 Grade B, A350 Grade LF3, and A105 Grade II steels 1.2 relative to that of similar materials irradiated at low temperatures in test reactors. ${ }^{3}$ The difference between the HFIR and test reactor data has been attributed to a fluence-rate effect, 1.e.. the degree of embrittlement per unit of fast fluence increases at low neutron flux, and/or to a softened neutron spectrum, 1.e., a high thermal-to-fast-neutron-flux ratio may contribute to accelerated embrittlement. 1 The relative contribution of fluence rate and spectrum could not be discerned because the effects were concurrent and inseparable. The HFIR surveillance specimens were exposed over a period of $\approx 17$ effective-full-power years (efpy) at $50^{\circ} \mathrm{C}\left(122^{\circ} \mathrm{F}\right)$ and a flux $(E>1 \mathrm{MeV})$ of $10^{8}-10^{9} \mathrm{n} / \mathrm{cm}^{2} \cdot \mathrm{s}$ to fluences of $10^{17}-10^{18} \mathrm{n} / \mathrm{cm}^{2}$. The fast-neutron flux for the HFIR surveillance samples was several orders of magnitude lower than that in test reactors $\left(10^{13} \mathrm{n} / \mathrm{cm}^{2} \cdot \mathrm{s}\right)$. At the surveillance position, thermal neutrons comprise $96 \%$ of the total flux. The possible effects of fluence rate or spectrum were validated by comparative tests on HFIR A212-B steel irradiated in the Oak Ridge Research Reactor (ORR) at a flux of $\approx 10^{13} \mathrm{n} / \mathrm{cm}^{2} \cdot \mathrm{s} .{ }^{1.2}$ The results indicated that an order of magnitude greater fluence is needed in the ORR to produce the same shift in Charpy transition temperature (CTT) that was observed in HFIR A212-B surveillance samples.

Current Nuclear Regulatory Commission (NRC) guidelines for the assessment of embrittlement of pressure vessel support structures of commercial LWRs do not consider the contributions of fluence rate or spectral effects. HFIR surveillance data raise the possibility that the guidelines may not be sufficiently conservative. 4 To help resolve this issue, a program was initiated to characterize the irradiation embrittlement of the neutron shield tank (NST) from the decommissioned Shippingport reactor. The Shippingport pressure vessel utilized the NST for its support structure. Shippingport was the first large-scale (72 MWe) nuclear power plant in the United States. Criticality was achieved in December 1957 and operation continued for a $25-y$ service life. Two separate pressurized-water reactor cores and one light-water breeder core were used during the life of the facility.

The NST support for the reactor pressure vessel is a skirt-mounted, annular, waterfilled tank that consists of two concentric shells extending above and below the reactor core. Fig. 1. It is exposed to reactor core beltline neutrons. Water is circulated through the tank for cooling and shielding. Shippingport NST operated at $55^{\circ} \mathrm{C}\left(130^{\circ} \mathrm{F}\right)$ and was fabricated from rolled A2 12 Grade B firebox steel similar to that used for the HFIR pressure vessel. The inner wall of the NST was exposed to a total maximum flucnce of $\approx 6 \times 10^{17} \mathrm{n} / \mathrm{cm}^{2}$ $(\mathrm{E}>1 \mathrm{MeV})$ over a life of $9.25 \mathrm{efpy}$. This corresponds to a fast-neutron flux of $\approx 2 \times 10^{9}$ $\mathrm{n} / \mathrm{cm}^{2}$.s. The temperature, fast fluence, and fast flux for the Shippingport NST were comparable to those for the HFIR surveillance samples.

\section{Material Characterization}

The effort to obtain samples from the NST was sponsored jointly by the NRC and the U.S. Department of Energy Plant Life Extension Program at Sandia National Laboratories. Sample removal was scheduled with the ongoing decommissioning operation that began in 


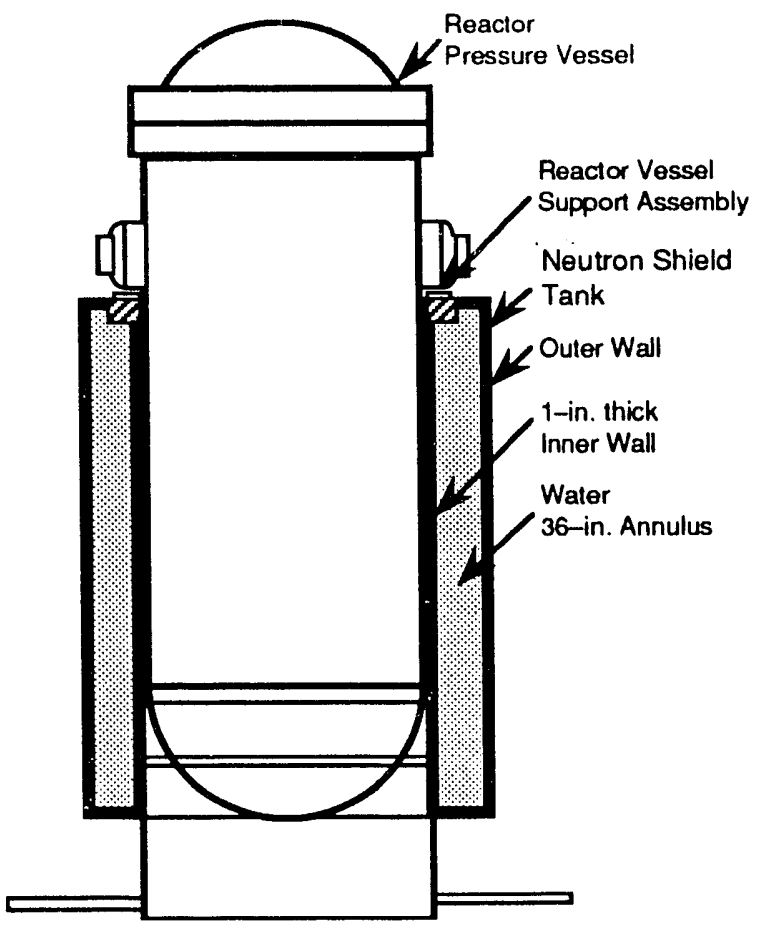

Figure 1. Schematic representation of the NST support for the Shippingport reactor pressure vessel
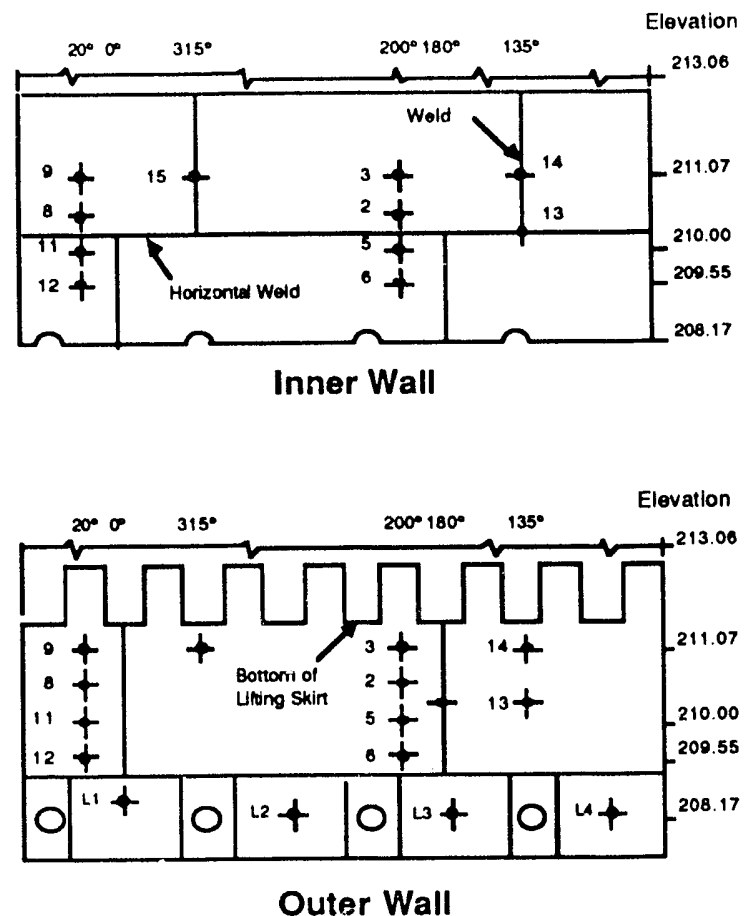

Figure 2. Layout of sample location in the Shippingport NST (a) inner and (b) outer walls

October 1982. The decommissioning plan called for a one-plece lift-out of the reactor pressure vessel and NST assembly following removal of fuel and core internals, and backfilling with a grouting material to serve as shielding. Cores were cut through the NST outer wall and grout material to gain access to the NST inner wall. The coring operation involved the combined use of a commercially available diamond bit and a hole saw to remove $\approx 155$ $\mathrm{mm}$ (6 in.) diameter samples from the inner and outer walls of the NST. The actual sampling was performed by personnel from Battelle Pacific Northwest Laboratory. A more detailed description of the sampling and of the program plan have been presented earlier. ${ }^{5,6}$ Eight samples of the base metal and three weld samples were obtained from the inner vall, along with corresponding samples from the outer wall. The layouts for the sample locations from the inner and outer walls are shown in Fig. 2.

The inner wall is constructed from four plates, each $\approx 25.4 \mathrm{~mm}(1 \mathrm{in}$.) thick. The two lower plates were joined by vertical full-penetration welus located along the azimuthal positions 0 and $180^{\circ}$ (north and south reference axes of the NST); the two upper plates were welded along the azimuthal positions of 135 and $315^{\circ}$. These two assemblies were joinc $d$ by a mid-height horizontal full-penetration weld. Specimen locations 13,14, and 15 in Fig. 2 contain vertical welds; the other locations represent the base metal from the four plates.

The outer wall was constructed from two plates joined by vertical welds at azimuthal positions 0 and $180^{\circ}$. A weld sample was obtained from the outer wall at Location 1 . All other samples from the outer wall represent base metal from the two plates. No radiation 
Table 1. Typical Composition (wt.\%) of plate and weld metal from the Shippingport NST

\begin{tabular}{llc}
\hline \multicolumn{1}{c}{ Element } & Plate & Weld \\
\hline $\mathrm{C}$ & 0.23 & 0.065 \\
$\mathrm{Mn}$ & 0.76 & 0.93 \\
$\mathrm{P}$ & 0.02 & - \\
$\mathrm{S}$ & 0.03 & - \\
$\mathrm{S}$ i & 0.27 & $0.73^{\mathrm{a}}$ \\
$\mathrm{Cu}$ & 0.05 & $0.06 \mathrm{~b}$ \\
$\mathrm{Ni}$ & 0.04 & 0.02 \\
$\mathrm{Cr}$ & 0.04 & 0.03 \\
$\mathrm{O}$ & 0.01 & - \\
$\mathrm{N}$ & 0.004 & - \\
$\mathrm{Ti}$ & $<0.005$ & 0.025 \\
$\mathrm{~V}$ & $<0.005$ & 0.019 \\
$\mathrm{Zr}$ & $<0.005$ & $<0.005$ \\
Mo. Ca, Al & $<0.01$ & $<0.01$ \\
Se. Sn, B & $<0.01$ & $<0.01$ \\
\hline a 0.86 for inner-wall weld from Location 14. \\
$\mathrm{b}$ 0.07 for outer-wall weld and 0.04 for inner-wall weld from \\
Location 15. &
\end{tabular}

effect is expected at the extremely low levels of fluence for the outer wall; therefore, the outer-wall samples were used to determine baseline data for nonirradiated samples. Samples from the lower portion of the NST outer wall, which had even lower fluence levels. i.e., Locations L1-L4, were also procured to establish baseline material properties.

Metallurgical characterization and chemical analyses of samples taken from each of the plates strongly suggest that both the inner and outer walls of the NST were fabricated from a single heat. Typical elemental composition of the plate and weld metal is given in Table 1. Metallographic examination of the NST material indicates that the rolling direction is aligned with the circumferential direction of the shield tank.

Micrographs of the grain structure of the Shippingport NST along the rolling and transverse sections are shown in Fig. 3. The surfaces shown in the micrographs are designated by the direction normal to the surface. The transverse section shows some elongated grains, and all the inclusions in the rolling direction are elongated. The inclusions in the rolling section are globular or flat. Average through-wall grain size was $\approx 12 \mu \mathrm{m}$, with no measurable change observed across the thickness of the wall.

Significant variations in hardness across the thickness of the NST wall were found at most locations. In general, the center of the plate is softer than the near-surface regions. A typical through-wall hardness profile (Rockwell $B, R_{B}$ ) for the outer wall is shown in Fig. 4. (The depth is measured from the inner surface of the plate, 1.e., the surface toward the reactor core.) Hardness ranges from $R_{B} 73$ to 83 , and hardness values of the innerand outer-7-mm regions of the wall are 5-10\% higher than those in the center of the wall. Although irradiation increases the hardness of the material, the V-shaped profile is maintained at most locations of the inner wall. The hardness profiles for Locations 3 and 9 of the NST inner wall are shown in Fig. 5. However, at a few locations of the inner and outer 


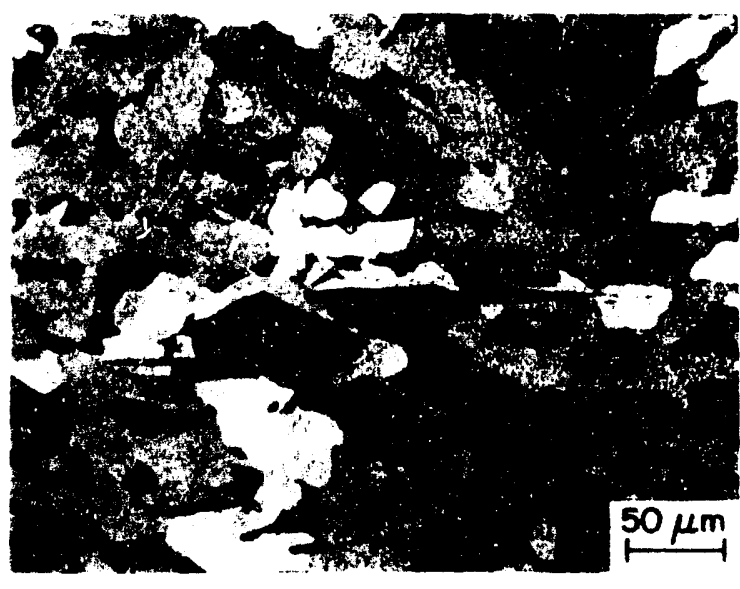

(a)

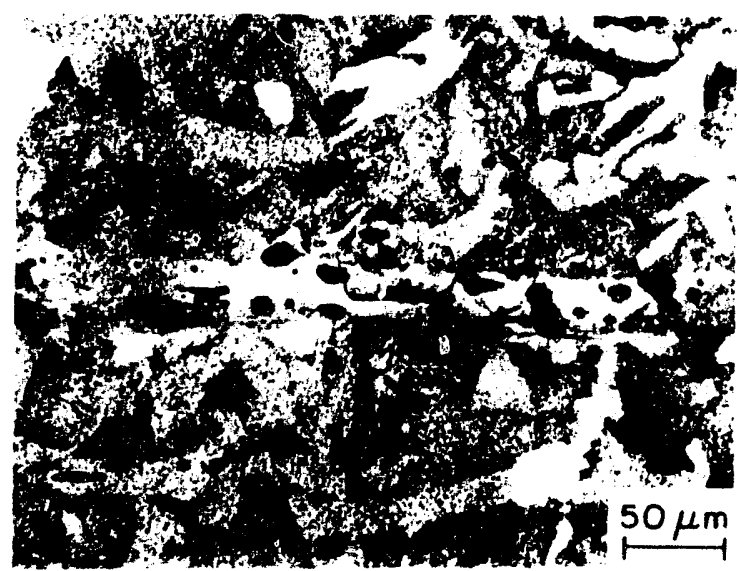

(b)

Figure 3. Micrographs of the Shippingport NST material along (a) transverse and (b) rolling sections

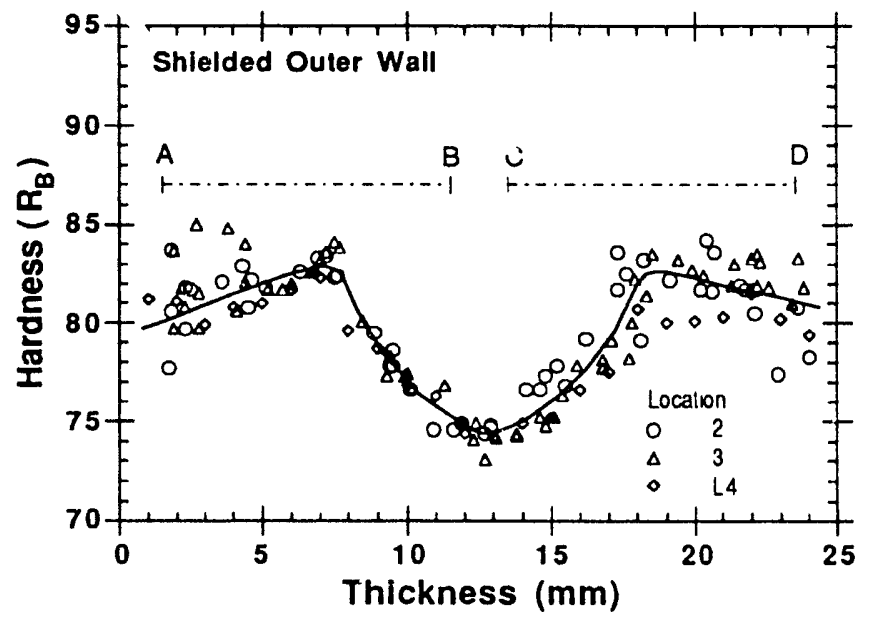

Figure 4.

Hardness profile across the thickness of the Shippingport NST outer wall. Regions $A-B$ and $C-D$ show the locations for Charpy-impact specimens

walls there was little or no variation in hardness across the wall thickness; an example is shown in Fig. 6 for inner-wall Location 8.

\section{Irradiation Embrittlement}

Irradiation embrittlement was characterized by Charpy-impact and tensile tests; the results have been presented earlier. ${ }^{7}$ Specimens were obtained in the longitudinal (LT) and transverse (TL) orientations ${ }^{*}$ from three $10-\mathrm{mm}$-wide regions (inner, center, and outer) across the thickness of the NST wall. To avoid confusion with regions, the NST inner and outer walls will be referred to as irradiated and shielded walls, respectively. The locations of Charpy-impact specimens from the inner- and outer-10-mm regions of the irradiated and shielded walls are shown in Figs. 4-6. Average hardness of the samples from the

\footnotetext{
* The first letter designates the direction normal to the plane of the crack, the second letter represents the direction of crack propagation. $\mathrm{L}=$ longitudinal or rolling direction and $\mathrm{T}=$ transverse direction.
} 

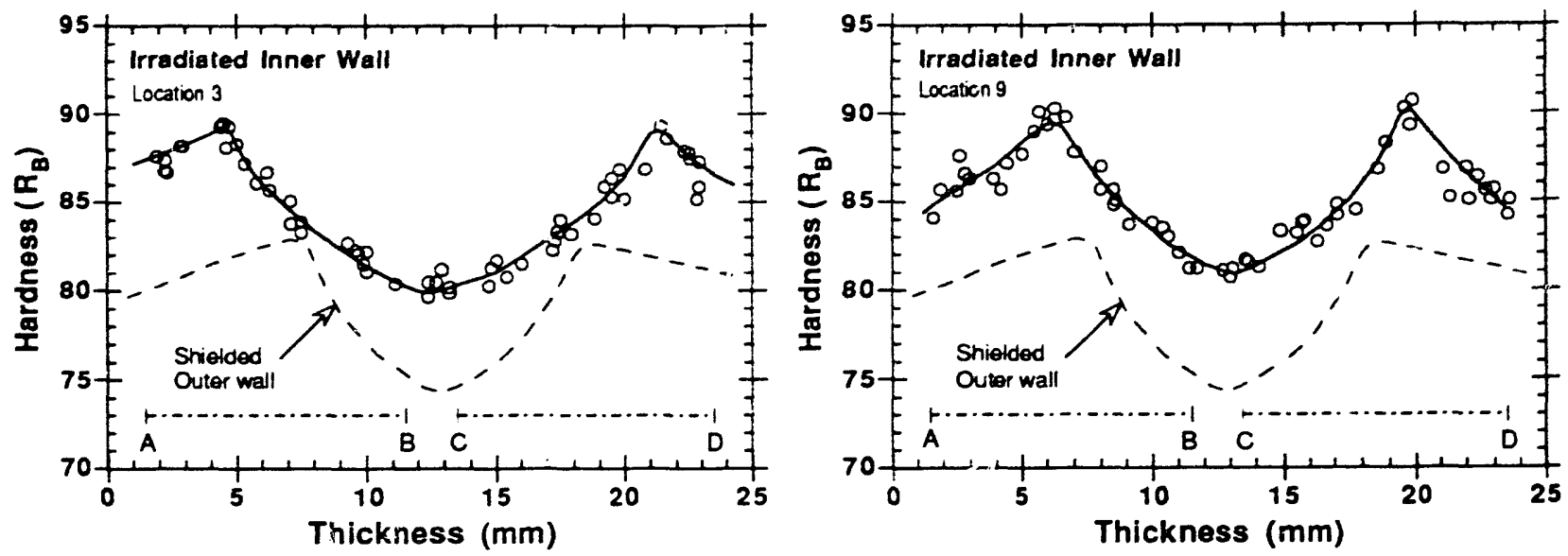

Figure 5. Hardness profiles for Locations 3 and 9 of the Shippingport NST inne; wall. Regions $A-B$ and $C-D$ show the locations for Charpy-impact specimens.

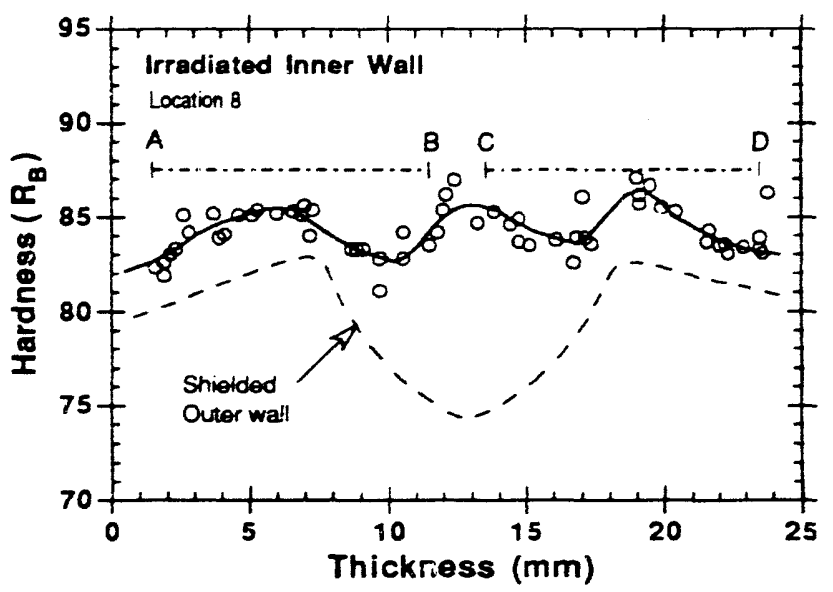

Figure 6.

Hardness profile for Location 8 of the Shippingport NST inner wall. Regions $A-B$ and $C-D$ show the locations for the Charpyimpact specimens.

inner- and outer-10-mm regions is approximately the same for all locations, whereas that of the center-10-mm region may be 5-10\% lower for some locations. Layouts for the basemetal and weld-metal specimens from the irradiated and shielded walls are shown in Fig. 7.

Total fluence is a function of sample location (Fig. 2): axial variation of fluence peaked at an elevation of $211.07 \mathrm{~m}(692.5 \mathrm{ft})$, and azimuthal variation of fluence peaked at the 20 and $200^{\circ}$ positions. The estimated fluence ${ }^{*}$ at the inner surface of the NST irradiated wall was $\approx 6 \times 10^{17} \mathrm{n} / \mathrm{cm}^{2}(\mathrm{E}>1 \mathrm{MeV})$ for Locations 3 and $9, \approx 4 \times 10^{17} \mathrm{n} / \mathrm{cm}^{2}$ for Locations 2 and $8, \approx 3 \times 10^{17} \mathrm{n} / \mathrm{cm}^{2}$ for Locations 14 and 15. and $\approx 2 \times 10^{17} \mathrm{n} / \mathrm{cm}^{2}$ for Location 13 . The data for activation measurements ${ }^{+}$indicate that the fluence decreases by a factor of 2-3 across the thickness of the NST irradiated wall. The fluences for the specimens from the outer$10-\mathrm{mm}$ region were estimated to be a factor of $\approx 1.5$ lower than those for specimens from the inner-10-mm region. Material irom the outer wall, which was protected by $\approx 0.9 \mathrm{~m}$ ( $3 \mathrm{ft}$ ) of wa ' $\mathrm{r}$ and hence had a fluence that was 6 orders of magnitude lower than that of the irradiated wall, was used to obtain baseline data for nonirradiated material.

\footnotetext{
- Jame, L., Bettis Atomic Power Laboratory, private communication (April 1989).

+ Greenwood, L.. Argonne National Laboratory, unpublished work (1989).
} 


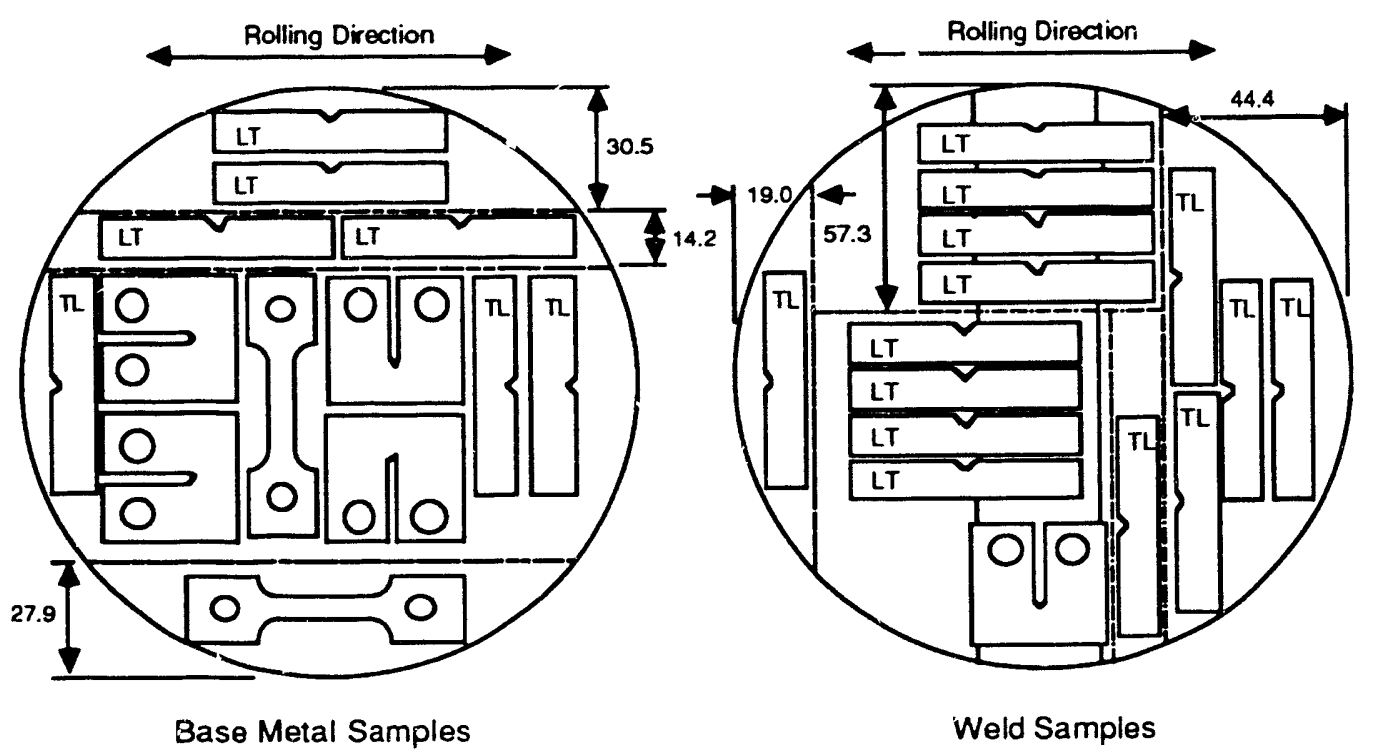

Figure 7. Cutting diagram for base-metal and weld-metal specimens fromi irradiated and shielded walls of the Shippingport NST

Charpy-impact tests were conducted on standard Charpy V-notch specimens machined according to ASTM Specification E 23. A Dynatup Model 8000A drop-weight impact machine with an insirumented tup and data readout system was used for the tests. Tensile tests were performed on dog-bone specimens with a cross section of $4 \times 5 \mathrm{~mm}$ $(0.16 \times 0.20 \mathrm{in}$.) and a gauge length of $20 \mathrm{~mm}(0.79 \mathrm{in}$.$) . The tests were conducted at an$ initial strain rate of $4 \times 10^{-4} \mathrm{~s}^{-1}$.

\subsection{Base Metal}

\subsubsection{Charpy-Impact Energy}

Results from Charpy-impact tests on LT and TL specimens from different regions and locations of the irradiated and shielded walls of the NST are given in Tables 2-4; best-fit Charpy transition curves are shown in Figs. 8-11. The Charpy data were fitted with a hyperbolic tangent function of the form

$$
\mathrm{CV}_{\mathrm{V}}=\mathrm{K}_{\mathrm{O}}+\mathrm{B}\{1+\tanh [(\mathrm{T}-\mathrm{C}) / \mathrm{D}]\}
$$

where $K_{0}$ is the lower-shelf energy. $T$ is the test temperature, $B$ is half the distance between upper- and lower-shelf energy, $C$ is the mid-shelf CTT in ${ }^{\circ} \mathrm{C}$, and D is the half-width of the transition region. The values of the constants $K_{0}, B, C$, and $D$ in Eq. 1, as well as the upper-shelf energy (USE) and the CTT at the 20.4 J (15 ft.lb) and $41-\mathrm{J}(30 \mathrm{ft} \cdot \mathrm{lb})$ levels for the various samples are given in Table 5.

The results for the shielded wall (Figs. 8 and 9) show no effect of sample location: there is little or no variation in the transition curves with vertical or azimuthal position. However, the TL orientation is weaker than the LT orientation. The CTT and USE, respectively, are $16^{\circ} \mathrm{C}\left(61^{\circ} \mathrm{F}\right)$ and $102 \mathrm{~J} / \mathrm{cm}^{2}(\approx 60 \mathrm{ft} \cdot \mathrm{lb})$ for $\mathrm{LT}$ specimens and $20^{\circ} \mathrm{C}\left(68^{\circ} \mathrm{F}\right)$ and 
Table 2. Charpy-impact data for the irradiated inner wall of the Shippingport NST

\begin{tabular}{|c|c|c|c|c|c|c|c|c|c|c|}
\hline \multirow{2}{*}{$\begin{array}{l}\text { Specimen } \\
\text { ID } \\
\end{array}$} & \multirow[b]{2}{*}{ Location } & \multirow[b]{2}{*}{ Regio: ${ }^{a}$} & \multirow{2}{*}{$\begin{array}{c}\text { Fluence }^{\mathrm{b}} \\
\left(\mathrm{n} / \mathrm{cm}^{2}\right)\end{array}$} & \multirow{2}{*}{$\begin{array}{l}\text { Orien- } \\
\text { tation }\end{array}$} & \multicolumn{2}{|c|}{ Test Te:np. } & \multicolumn{2}{|c|}{ Impact Energy } & \multicolumn{2}{|c|}{ Load $(\mathrm{kN})$} \\
\hline & & & & & $\left({ }^{\circ} \mathrm{C}\right)$ & $\left({ }^{\circ} \mathrm{F}\right)$ & $\left(\mathrm{J} / \mathrm{cm}^{2}\right)$ & $(\mathrm{ft} \cdot \mathrm{lb})$ & Yield & Maximum \\
\hline $\begin{array}{l}\text { I03R-05 } \\
\text { I03R-01 }\end{array}$ & $\begin{array}{l}3 \\
3\end{array}$ & $\begin{array}{l}\text { I } \\
\text { I }\end{array}$ & $6 \times 10^{17}$ & $\begin{array}{l}\text { LT } \\
\text { LT }\end{array}$ & $\begin{array}{l}10 \\
25\end{array}$ & $\begin{array}{l}50 \\
77\end{array}$ & $\begin{array}{r}8.4 \\
10.9\end{array}$ & $\begin{array}{l}5.0 \\
6.4\end{array}$ & $\begin{array}{l}8.788 \\
9.872\end{array}$ & $\begin{array}{l}8.788 \\
9.872\end{array}$ \\
\hline I03R-03 & 3 & I & & LT & 55 & 131 & 45.5 & 26.8 & 12.313 & 14.198 \\
\hline 103R-07 & 3 & 1 & & LT & 70 & 158 & 68.8 & 40.6 & 12.303 & 13.934 \\
\hline $103 R-08$ & 3 & o & & LT & 0 & 32 & 3.7 & 2.2 & 10.194 & 10.194 \\
\hline I03R-02 & 3 & 0 & & LT & 25 & 77 & 14.5 & 8.6 & 12.411 & 12.411 \\
\hline I03R-06 & 3 & 0 & & iT & 40 & 104 & 25.2 & 15.5 & 12.254 & 12.254 \\
\hline $103 R-04$ & 3 & 0 & & LT & 55 & 131 & 61.0 & 36.0 & 11.356 & 13.866 \\
\hline $103 \mathrm{~T}-03$ & 3 & 1 & & $T L$ & 120 & 248 & 51.3 & 30.3 & 10.428 & 11.327 \\
\hline I03T-02 & 3 & 0 & & TL & 25 & 77 & 10.0 & 5.9 & 10.389 & 10.389 \\
\hline I03T.04 & 3 & 0 & & TL & 70 & 158 & 40.6 & 24.0 & 11.EiO & 12.303 \\
\hline 109R-05 & 9 & 1 & $6 \times 10^{17}$ & LT & 10 & 50 & 7.1 & 4.2 & 8.983 & 8.983 \\
\hline 109R-01 & 9 & 1 & & LT & 25 & 77 & 8.1 & 4.8 & 11.844 & 11.844 \\
\hline IOSR-03 & 9 & I & & LT & 55 & 131 & 39.0 & 23.0 & 12.401 & 13.866 \\
\hline 109R-07 & 9 & I & & LT & 70 & 158 & 51.7 & 30.5 & 12.518 & 13.856 \\
\hline $109 R-08$ & 9 & 0 & & LT & 0 & 32 & 3.1 & 1.8 & 7.392 & 7.392 \\
\hline I09R-02 & 9 & O & & LT & 25 & 77 & 11.3 & 6.7 & 11.044 & 11.044 \\
\hline I09R-06 & 9 & O & & LT & 40 & 104 & 31.3 & 18.5 & 12.391 & 12.391 \\
\hline I09R-04 & 9 & 0 & & LT & 55 & 131 & 48.5 & 28.6 & 12.254 & 14.256 \\
\hline $109 \mathrm{~T}-03$ & 9 & I & & TL & 40 & 104 & 19.1 & 11.3 & 10.194 & 10.194 \\
\hline $109 \mathrm{~T}-01$ & 9 & I & & $T L$ & 55 & 131 & 28.0 & 16.5 & 12.782 & 13.123 \\
\hline $109 T-02$ & 9 & O & & TL & 25 & 77 & 9.6 & 5.7 & 11.786 & 11.786 \\
\hline IO9T-04 & 9 & 0 & & $T L$ & 90 & 194 & 53.0 & 31.3 & 11.424 & 12.411 \\
\hline $102 R-05$ & 2 & 1 & $4 \times 10^{17}$ & LT & 10 & 50 & 9.9 & 5.8 & 9.120 & 9.120 \\
\hline $102 R-01$ & 2 & 1 & & LT & 25 & 77 & 13.5 & 8.0 & 10.331 & 10.331 \\
\hline IO2R-03 & 2 & 1 & & LT & 55 & 131 & 52.8 & 31.2 & 11.688 & 13.260 \\
\hline I02R-07 & 2 & 1 & & LT & 70 & 158 & 67.7 & 39.9 & 11.678 & 13.612 \\
\hline I02R-08 & 2 & 0 & & LT & 0 & 32 & 22.7 & 13.4 & 11.854 & 11.854 \\
\hline IO2R-02 & 2 & $\mathrm{O}$ & & LT & 25 & 77 & 11.6 & 6.8 & 12.616 & 12.616 \\
\hline $102 R-06$ & 2 & 0 & & LT & 40 & 104 & 28.5 & 16.8 & 10.975 & 10.975 \\
\hline IO2R-04 & 2 & $\mathrm{O}$ & & LT & 55 & 131 & 68.6 & 40.5 & 11.190 & 13.670 \\
\hline $102 \mathrm{~T}-01$ & 2 & 1 & & $T L$ & 55 & 131 & 40.9 & 24.1 & 11.639 & 12.186 \\
\hline $102 T-03$ & 2 & $i$ & & TL & 120 & 248 & 53.3 & 31.4 & 9.989 & 11.317 \\
\hline $102 \mathrm{~T}-02$ & 2 & 0 & & TL & 25 & 77 & 12.4 & 7.3 & 12.821 & 12.821 \\
\hline I02T-04 & 2 & O & & $T L$ & 70 & 158 & 49.4 & 29.1 & 10.966 & 12.059 \\
\hline 108R-05 & 8 & 1 & $4 \times 10^{17}$ & LT & 10 & 50 & 9.5 & 5.6 & 13.387 & 13.387 \\
\hline I08R-01 & 8 & I & & LT & 25 & 77 & 10.3 & 6.1 & 9.647 & 9.647 \\
\hline IO8R-03 & 8 & 1 & & LT & 55 & 131 & 43.5 & 25.7 & 12.381 & 14.188 \\
\hline I08R-07 & 8 & 1 & & LT & 70 & 158 & 79.7 & 47.0 & 11.747 & 13.729 \\
\hline $108 \mathrm{R}-08$ & 8 & 0 & & LT & 0 & 32 & 3.1 & 1.8 & 8.935 & 8.935 \\
\hline $108 \mathrm{R}-02$ & 8 & 0 & & LT & 25 & 77 & 12.8 & 7.6 & 12.811 & 12.811 \\
\hline 108R-06 & 8 & 0 & & LT & 40 & 104 & 26.8 & 15.8 & 12.391 & 12.391 \\
\hline $108 \mathrm{R}-04$ & 8 & $\mathrm{O}$ & & LT & 55 & 131 & 53.5 & 31.6 & 11.962 & 13.993 \\
\hline $108 \mathrm{~T}-03$ & 8 & I & & TL & 40 & 104 & 24.8 & 14.6 & 12.635 & 13.026 \\
\hline $108 \mathrm{~T}-01$ & 8 & I & & TL & 55 & 131 & 40.7 & 24.0 & 11.913 & 12.635 \\
\hline $108 \mathrm{~T}-02$ & 8 & 0 & & TL & 25 & 77 & 12.3 & 7.3 & 13.221 & 13.221 \\
\hline $108 \mathrm{~T}-04$ & 8 & $\mathrm{O}$ & & TL & 90 & 194 & 49.7 & 29.3 & 10.995 & 11.874 \\
\hline $114 \mathrm{~T}-01 \mathrm{C}$ & 14 & C & $3 \times 10^{17}$ & TL & 0 & 32 & 5.5 & 3.2 & 8.524 & 8.524 \\
\hline I $14 \mathrm{~T}-02 \mathrm{C}$ & 14 & C & & TL & 25 & 77 & 12.8 & 7.6 & 9.374 & 9.374 \\
\hline $114 \mathrm{~T}-03 \mathrm{C}$ & 14 & C & & $T L$ & 55 & 131 & 36.8 & 21.7 & 11.502 & 11.502 \\
\hline $114 \mathrm{~T}-04 \mathrm{C}$ & 14 & C & & $T L$ & 90 & 194 & 55.8 & 32.9 & 10.253 & 11.288 \\
\hline $114 \mathrm{~T}-02$ & 14 & $\mathrm{O}$ & & $T L$ & 90 & 194 & 54.8 & 32.3 & 10.643 & 11.835 \\
\hline $115 \mathrm{~T}-01 \mathrm{C}$ & 15 & C & $3 \times 10^{17}$ & $\mathrm{TL}$ & 10 & 50 & 8.4 & 5.0 & 8.476 & 8.476 \\
\hline $115 \mathrm{~T}-02 \mathrm{C}$ & 15 & $\mathrm{C}$ & & $\mathrm{TL}$ & 40 & 104 & 27.0 & 15.9 & 11.512 & 11.512 \\
\hline $115 T-03 C$ & 15 & $\mathrm{C}$ & & $\mathrm{TL}$ & 70 & 158 & 40.2 & 23.7 & 10.721 & 10.917 \\
\hline $115 \mathrm{~T}-04 \mathrm{C}$ & 15 & $\mathrm{C}$ & & $\mathrm{TL}$ & 120 & 248 & 46.2 & 27.3 & 9.511 & 10.321 \\
\hline
\end{tabular}


Table 2. (Contd.)

\begin{tabular}{|c|c|c|c|c|c|c|c|c|c|c|}
\hline \multirow{2}{*}{$\begin{array}{c}\text { Specimen } \\
\text { ID }\end{array}$} & \multirow[b]{2}{*}{ Location } & \multirow{2}{*}{ Regiona } & \multirow{2}{*}{$\begin{array}{c}\text { Fluenceb } \\
\left(\mathrm{n} / \mathrm{cm}^{2}\right)\end{array}$} & \multirow{2}{*}{$\begin{array}{l}\text { Orien- } \\
\text { tation }\end{array}$} & \multicolumn{2}{|c|}{ Test Temp. } & \multicolumn{2}{|c|}{ Impact Energy } & \multicolumn{2}{|c|}{ Load $(\mathrm{kN})$} \\
\hline & & & & & $\left({ }^{\circ} \mathrm{C}\right)$ & $\left({ }^{\circ} \mathrm{F}\right)$ & $\left(\mathrm{J} / \mathrm{cm}^{2}\right)$ & $(\mathrm{ft} \cdot \mathrm{lb})$ & Yield & Maximum \\
\hline $115 \mathrm{~T}-01$ & 15 & I & $3 \times 10^{17}$ & TI & 40 & 104 & 21.1 & 12.5 & 12.665 & 12.665 \\
\hline I13T-02 & 13 & 0 & $2 \times 10^{17}$ & $\mathrm{TL}$ & 25 & 77 & 10.1 & 6.0 & 9.325 & 9.325 \\
\hline
\end{tabular}

Table 3. Charpy-impact data for the shielded outer wall of the Shippingport NST

\begin{tabular}{|c|c|c|c|c|c|c|c|c|c|}
\hline \multirow{2}{*}{$\begin{array}{c}\text { Specimen } \\
\text { ID }\end{array}$} & \multirow[b]{2}{*}{ Location } & \multirow[b]{2}{*}{ Regiona } & \multirow{2}{*}{$\begin{array}{l}\text { Orien- } \\
\text { tation }\end{array}$} & \multicolumn{2}{|c|}{ Test Temp. } & \multicolumn{2}{|c|}{ Impact Energy } & \multicolumn{2}{|c|}{ Load $(\mathrm{kN})$} \\
\hline & & & & $\left({ }^{\circ} \mathrm{C}\right)$ & $\left({ }^{\circ} \mathrm{F}\right)$ & $\left(\mathrm{N} / \mathrm{cm}^{2}\right)$ & (ft.lb) & Yield & Maximum \\
\hline OO2R-05 & 2 & 1 & LT & 10 & 50 & 18.7 & 11.0 & 10.448 & 10.448 \\
\hline OO2R-01 & 2 & I & LT & 25 & 77 & 29.4 & 17.3 & 11.971 & 12.704 \\
\hline OO2R-03 & 2 & I & LT & 55 & 131 & 114.3 & 67.4 & 10.692 & 14.530 \\
\hline OO2R-07 & 2 & I & LT & 70 & 158 & 95.5 & 56.3 & 10.917 & 13.651 \\
\hline OO2R-08 & 2 & $\mathrm{O}$ & LT & 0 & 32 & 11.9 & 7.0 & 11.239 & 11.239 \\
\hline O2R-02 & 2 & 0 & LT & 25 & 77 & 48.0 & 28.3 & 11.522 & 13.934 \\
\hline O02R-06 & 2 & 0 & $\mathrm{LT}$ & 40 & 104 & 71.2 & 42.0 & 10.497 & 13.358 \\
\hline O02R-04 & 2 & 0 & LT & 55 & 131 & 92.0 & 54.3 & 10.692 & 13.973 \\
\hline O02T-03 & 2 & 1 & TL & 0 & 32 & 11.1 & 6.5 & 10.878 & 10.878 \\
\hline O02T-01 & 2 & 1 & $\mathrm{TL}$ & 55 & 131 & 57.0 & 33.6 & 11.083 & 12.939 \\
\hline $002 \mathrm{~T}-02$ & 2 & $\mathrm{O}$ & TL & 25 & 77 & 31.2 & 18.4 & 12.040 & 12.870 \\
\hline O02T-04 & 2 & $\mathrm{O}$ & TL & 90 & 194 & 69.8 & 41.2 & 10.145 & 12.284 \\
\hline OO3R-05 & 3 & I & $L T$ & 10 & 50 & 17.0 & 10.0 & 10.497 & 10.497 \\
\hline O03R-01 & 3 & I & LT & 25 & 77 & 35.1 & 20.7 & 11.207 & 12.186 \\
\hline OO3R-03 & 3 & 1 & LT & 55 & 131 & 94.8 & 55.9 & 10.155 & 13.592 \\
\hline O03R-07 & 3 & 1 & LT & 70 & 158 & 102.4 & 60.4 & 10.780 & 14.022 \\
\hline O03R-08 & 3 & 0 & $\mathrm{LT}$ & 0 & 32 & 10.7 & 6.3 & 10.526 & 10.526 \\
\hline O03R-02 & 3 & 0 & LT & 25 & 77 & 48.0 & 28.3 & 10.975 & 13.690 \\
\hline O03R-06 & 3 & 0 & LT & 40 & 104 & 61.1 & 36.1 & 11.405 & 14.032 \\
\hline OO3R-04 & 3 & 0 & LT & 55 & 131 & 98.3 & 58.0 & 10.214 & 13.680 \\
\hline 003T-03 & 3 & I & $T L$ & 10 & 50 & 18.0 & 10.6 & 12.713 & 12.713 \\
\hline O03T-01 & 3 & 1 & TI. & 55 & 131 & 53.6 & 31.6 & 11.376 & 13.241 \\
\hline O03T-02 & 3 & $\mathrm{O}$ & $\mathrm{TL}$ & 25 & 77 & 31.3 & 18.5 & 12.088 & 12.694 \\
\hline OO3T-04 & 3 & 0 & $T L$ & 70 & 158 & 68.9 & 40.7 & 10.497 & 12.518 \\
\hline 003T-06 & 3 & 0 & TL & 120 & 248 & 68.0 & 40.1 & 9.276 & 11.522 \\
\hline O06R-05 & 6 & I & LT & 10 & 50 & 14.2 & 8.4 & 10.887 & 10.887 \\
\hline O06R-01 & 6 & I & LT & 25 & 77 & 24.0 & 14.2 & 11.522 & 11.522 \\
\hline O06R-03 & 6 & i & LT & 55 & 131 & 89.1 & 52.6 & 10.594 & 14.051 \\
\hline O06R-07 & 6 & i & $\mathrm{LT}$ & 70 & 158 & 89.5 & 52.8 & 10.887 & 13.914 \\
\hline O06R-08 & 6 & 0 & $\mathrm{LT}$ & 0 & 32 & 13.2 & 7.8 & 9.110 & 9.110 \\
\hline C06R-02 & 6 & 0 & LT & 25 & 77 & 42.5 & 25.1 & 11.395 & 13.465 \\
\hline 006R-06 & 6 & 0 & LT & 40 & 104 & 50.4 & 29.7 & 11.581 & 13.163 \\
\hline O06R-04 & 6 & 0 & LT & 55 & 131 & 93.3 & 55.1 & 10.477 & 13.846 \\
\hline O06T-03 & 6 & I & $T L$ & 40 & 104 & 42.4 & 25.0 & 11.405 & 12.528 \\
\hline O06T-01 & 6 & I & $T L$ & 55 & 131 & 56.7 & 33.5 & 11.268 & 12.831 \\
\hline 006T-05 & 6 & 1 & $T^{L}$ & 90 & 194 & 69.8 & 41.2 & 9.862 & 12.206 \\
\hline O06T-04 & 6 & 0 & $T L$ & 0 & 32 & 13.4 & 7.9 & 13.075 & 13.075 \\
\hline O06T-02 & 6 & $\mathrm{O}$ & TL & 25 & 77 & 24.7 & 14.6 & 12.860 & 12.860 \\
\hline OO9R-05 & 9 & 1 & $L T$ & 10 & 50 & 26.2 & 15.5 & 12.479 & 12.479 \\
\hline OO9R-01 & 9 & 1 & LT & 25 & 77 & 43.2 & 25.5 & 11.190 & 11.971 \\
\hline OO9R-03 & 9 & I & LT & 55 & 131 & 85.7 & 50.6 & 10.614 & 12.381 \\
\hline O09R-07 & 9 & 1 & LT & 70 & 158 & 88.0 & 51.9 & 10.272 & 12.635 \\
\hline OO9R-08 & 9 & 0 & LT & 0 & 32 & 16.5 & 9.7 & 12.987 & 12.987 \\
\hline O09R-02 & 9 & 0 & LT & 25 & 77 & 46.4 & 27.4 & 11.278 & 11.883 \\
\hline O09R-06 & 9 & 0 & LT & 40 & 104 & 67.5 & 39.8 & 11.171 & 12.215 \\
\hline
\end{tabular}


Table 3. (Contd.).

\begin{tabular}{|c|c|c|c|c|c|c|c|c|c|}
\hline \multirow{2}{*}{$\begin{array}{c}\text { Specimen } \\
\text { ID } \\
\end{array}$} & \multirow[b]{2}{*}{ Location } & \multirow[b]{2}{*}{ Region $^{a}$} & \multirow{2}{*}{$\begin{array}{c}\text { Orien- } \\
\text { tation }\end{array}$} & \multicolumn{2}{|c|}{ Test Temp. } & \multicolumn{2}{|c|}{ Impact Energy } & \multicolumn{2}{|c|}{ Load $(\mathrm{kN})$} \\
\hline & & & & $\left({ }^{\circ} \mathrm{C}\right)$ & $\left({ }^{\circ} \mathrm{F}\right)$ & $\left(\mathrm{J} / \mathrm{cm}^{2}\right)$ & $(\mathrm{ft} \cdot \mathrm{lb})$ & Yield & Maximum \\
\hline OD9R-04 & 9 & $\mathrm{O}$ & LT & 55 & 131 & 87.7 & 51.7 & 10.057 & 12.430 \\
\hline $\begin{array}{l}\text { O09Т-03 } \\
\text { O09Т-01 } \\
\text { О09Т-02 } \\
\text { O09Т-04 } \\
\text { O09Т-06 }\end{array}$ & $\begin{array}{l}9 \\
9 \\
9 \\
9 \\
9\end{array}$ & $\begin{array}{l}1 \\
1 \\
0 \\
0 \\
0\end{array}$ & $\begin{array}{l}\text { TL } \\
T L \\
T L \\
T L \\
T L\end{array}$ & $\begin{array}{r}10 \\
55 \\
25 \\
70 \\
120\end{array}$ & $\begin{array}{r}50 \\
131 \\
77 \\
158 \\
248\end{array}$ & $\begin{array}{l}30.0 \\
52.5 \\
36.5 \\
58.2 \\
56.9\end{array}$ & $\begin{array}{l}17.7 \\
31.0 \\
21.5 \\
34.3 \\
33.6\end{array}$ & $\begin{array}{r}12.098 \\
10.565 \\
12.342 \\
9.804 \\
8.505\end{array}$ & $\begin{array}{l}12.098 \\
11.454 \\
12.342 \\
11.180 \\
10.555\end{array}$ \\
\hline $\begin{array}{l}\text { O12R-05 } \\
\text { O12R-03 } \\
\text { O12R-07 } \\
\text { O12R-08 } \\
\text { O12R-02 } \\
\text { O12R-06 } \\
\text { O12R-04 }\end{array}$ & $\begin{array}{l}12 \\
12 \\
12 \\
12 \\
12 \\
12 \\
12\end{array}$ & $\begin{array}{l}1 \\
1 \\
1 \\
0 \\
0 \\
0 \\
0\end{array}$ & $\begin{array}{l}\text { LT } \\
\text { LT } \\
\text { LT } \\
\text { LT } \\
\text { LT } \\
\text { LT } \\
\text { LT }\end{array}$ & $\begin{array}{r}10 \\
55 \\
70 \\
0 \\
25 \\
40 \\
55\end{array}$ & $\begin{array}{r}50 \\
131 \\
158 \\
32 \\
77 \\
104 \\
131\end{array}$ & $\begin{array}{l}19.0 \\
84.0 \\
91.3 \\
15.8 \\
49.4 \\
66.3 \\
96.5\end{array}$ & $\begin{array}{r}11.2 \\
49.6 \\
53.9 \\
9.3 \\
29.1 \\
39.1 \\
56.9\end{array}$ & $\begin{array}{r}12.616 \\
10.292 \\
10.468 \\
10.184 \\
11.083 \\
11.288 \\
9.843\end{array}$ & $\begin{array}{l}12.616 \\
12.782 \\
12.967 \\
10.184 \\
11.796 \\
12.625 \\
13.114\end{array}$ \\
\hline $\begin{array}{l}012 \mathrm{~T}-03 \\
012 \mathrm{~T}-01 \\
012 \mathrm{~T}-05 \\
\mathrm{O} 12 \mathrm{~T}-04 \\
012 \mathrm{~T}-02\end{array}$ & $\begin{array}{l}12 \\
12 \\
12 \\
12 \\
12\end{array}$ & $\begin{array}{l}I \\
I \\
I \\
O \\
O\end{array}$ & $\begin{array}{l}\text { TL } \\
\text { TL } \\
\text { TL } \\
\text { TL } \\
\text { TL }\end{array}$ & $\begin{array}{r}40 \\
55 \\
90 \\
0 \\
25\end{array}$ & $\begin{array}{r}104 \\
131 \\
194 \\
32 \\
77\end{array}$ & $\begin{array}{r}39.5 \\
57.9 \\
62.4 \\
8.9 \\
21.7\end{array}$ & $\begin{array}{r}23.3 \\
34.2 \\
36.8 \\
5.3 \\
12.8\end{array}$ & $\begin{array}{r}11.444 \\
10.341 \\
9.462 \\
10.262 \\
12.137\end{array}$ & $\begin{array}{l}12.206 \\
11.581 \\
11.366 \\
10.262 \\
12.137\end{array}$ \\
\hline $\begin{array}{l}\text { O14R-01C } \\
\text { O14R-05C } \\
\text { O14R-02C } \\
\text { O14R-06C } \\
\text { O14R-04C } \\
\text { O14R-03C } \\
\text { O14R-01 } \\
\text { O14R-02 }\end{array}$ & $\begin{array}{l}14 \\
14 \\
14 \\
14 \\
14 \\
14 \\
14 \\
14\end{array}$ & $\begin{array}{l}C \\
C \\
C \\
C \\
C \\
C \\
I \\
O\end{array}$ & $\begin{array}{l}\text { LT } \\
\text { LT } \\
\text { LT } \\
\text { LT } \\
\text { LT } \\
\text { LT } \\
\text { LT } \\
\text { LT }\end{array}$ & $\begin{array}{r}0 \\
25 \\
40 \\
55 \\
70 \\
90 \\
40 \\
90\end{array}$ & $\begin{array}{r}32 \\
77 \\
104 \\
131 \\
158 \\
194 \\
104 \\
194\end{array}$ & $\begin{array}{r}19.9 \\
58.7 \\
84.6 \\
100.3 \\
114.3 \\
103.1 \\
58.4 \\
102.5\end{array}$ & $\begin{array}{l}11.7 \\
34.6 \\
49.9 \\
59.2 \\
67.4 \\
60.8 \\
34.5 \\
60.5\end{array}$ & $\begin{array}{r}12.001 \\
10.663 \\
10.175 \\
9.667 \\
9.403 \\
8.495 \\
11.288 \\
9.999\end{array}$ & $\begin{array}{l}12.001 \\
12.586 \\
12.704 \\
12.420 \\
12.616 \\
11.464 \\
13.514 \\
12.831\end{array}$ \\
\hline $\begin{array}{l}\text { O14T-01C } \\
\text { O14T-03C } \\
\text { O14T-04C } \\
\text { O14T-02C }\end{array}$ & $\begin{array}{l}14 \\
14 \\
14 \\
14\end{array}$ & $\begin{array}{l}C \\
C \\
C \\
C\end{array}$ & $\begin{array}{l}\text { TL } \\
\text { TL } \\
\text { TL } \\
\text { TL }\end{array}$ & $\begin{array}{l}10 \\
40 \\
55 \\
90\end{array}$ & $\begin{array}{r}50 \\
104 \\
131 \\
194\end{array}$ & $\begin{array}{l}35.8 \\
64.0 \\
68.5 \\
73.3\end{array}$ & $\begin{array}{l}21.1 \\
37.8 \\
40.4 \\
43.3\end{array}$ & $\begin{array}{r}12.157 \\
9.950 \\
9.520 \\
8.895\end{array}$ & $\begin{array}{l}12.157 \\
12.069 \\
12.079 \\
11.532\end{array}$ \\
\hline $\begin{array}{l}\text { O15R-01C } \\
\text { O15R-02C } \\
\text { O15R-05C } \\
\text { O15R-03C } \\
\text { O15R-06C } \\
\text { O15R-04C } \\
\text { O15R-01 }\end{array}$ & $\begin{array}{l}15 \\
15 \\
15 \\
15 \\
15 \\
15 \\
15\end{array}$ & $\begin{array}{l}C \\
C \\
C \\
C \\
C \\
C \\
I\end{array}$ & $\begin{array}{l}\text { LT } \\
\text { LT } \\
\text { LT } \\
\text { IT } \\
\text { LT } \\
\text { LT } \\
\text { LT }\end{array}$ & $\begin{array}{r}10 \\
25 \\
40 \\
55 \\
80 \\
120 \\
-20\end{array}$ & $\begin{array}{r}50 \\
77 \\
104 \\
131 \\
176 \\
248 \\
-4\end{array}$ & $\begin{array}{r}19.3 \\
51.8 \\
77.4 \\
79.8 \\
105.4 \\
95.1 \\
6.4\end{array}$ & $\begin{array}{r}11.4 \\
30.6 \\
45.7 \\
4.7 .1 \\
62.2 \\
56.1 \\
3.8\end{array}$ & $\begin{array}{r}12.372 \\
11.337 \\
10.887 \\
10.555 \\
10.048 \\
8.866 \\
10.879\end{array}$ & $\begin{array}{l}12.372 \\
14.422 \\
13.914 \\
13.729 \\
13.573 \\
12.362 \\
10.879\end{array}$ \\
\hline $\begin{array}{l}\text { O15T-04C } \\
\text { O15T-01C } \\
\text { O15T-02C } \\
\text { O15T-03C }\end{array}$ & $\begin{array}{l}15 \\
15 \\
15 \\
15\end{array}$ & $\begin{array}{l}C \\
C \\
C \\
C\end{array}$ & $\begin{array}{l}\text { TL } \\
\text { TL } \\
\text { TL } \\
\text { TL }\end{array}$ & $\begin{array}{r}0 \\
25 \\
25 \\
70\end{array}$ & $\begin{array}{r}32 \\
77 \\
77 \\
158\end{array}$ & $\begin{array}{l}29.4 \\
42.3 \\
46.3 \\
76.6\end{array}$ & $\begin{array}{l}17.3 \\
25.0 \\
27.3 \\
45.2\end{array}$ & $\begin{array}{r}12.440 \\
11.688 \\
11.405 \\
9.823\end{array}$ & $\begin{array}{l}14.129 \\
13.885 \\
14.285 \\
13.387\end{array}$ \\
\hline
\end{tabular}

a I. O, and $C$ represent the inner-, outer-, and center-10-mm regions, respectively, across the wall thickness.

$67 \mathrm{~J} / \mathrm{cm}^{2}(\approx 40 \mathrm{ft} \cdot \mathrm{lb})$ for TL specimens. The differences in impact strength for the two ordentations are attributed primarily to differences in the distribution of inclusions along the crack plane. The plane of the crack for the TL orientation, 1.e., the transverse section shown in Fig. 3, contains elongated inclusions.

The results also indicate some effect of position through the thickness of the wall: impact energies for specimens from the inner and outer regions of the wall are comparable. whereas those for the center specimens are slightly higher. The CTT and USE for the center specimens are, respectively, $9^{\circ} \mathrm{C}\left(48^{\circ} \mathrm{F}\right)$ and $103 \mathrm{~J} / \mathrm{cm}^{2}(\approx 61 \mathrm{ft} \cdot \mathrm{lb})$ for the LT orientation 
Table 4. Charpy-impact data for Location L4 of the shielded outer wall of the Shippingport NST

\begin{tabular}{|c|c|c|c|c|c|c|c|}
\hline \multirow{2}{*}{$\begin{array}{l}\text { Specimen } \\
\text { ID } \\
\end{array}$} & \multirow[b]{2}{*}{ Location } & \multirow[b]{2}{*}{ Region ${ }^{a}$} & \multirow{2}{*}{$\begin{array}{l}\text { Orien- } \\
\text { tation }\end{array}$} & \multicolumn{2}{|c|}{ Test Temp. } & \multicolumn{2}{|c|}{ Impact Energy } \\
\hline & & & & $\left({ }^{\circ} \mathrm{C}\right)$ & $\left({ }^{\circ} \mathrm{F}\right)$ & $\left(\mathrm{J} / \mathrm{cm}^{2}\right)$ & $(\mathrm{ft} \cdot \mathrm{lb})$ \\
\hline$L A I-1$ & $\mathrm{~L} A$ & 1 & LT & -40 & -40 & 6.8 & 4.0 \\
\hline $40-6$ & LA & 0 & LT & -40 & -40 & 2.5 & 1.5 \\
\hline LAI -7 & LA & $I$ & LT & 0 & 32 & 5.9 & 3.5 \\
\hline $\mathrm{LAO}_{2}$ & LA & $\mathrm{O}$ & LT & 0 & 32 & 5.1 & 3.0 \\
\hline LAI -2 & LA & I & LT & 15 & -9 & 22.0 & 13.0 \\
\hline $\mathrm{LAO}-3$ & $\mathrm{~L} A$ & $\mathrm{O}$ & LT & 15 & -9 & 20.3 & 12.0 \\
\hline LAI -3 & LA & I & LT & 25 & 77 & 60.2 & 35.5 \\
\hline $\mathrm{LAO}-1$ & LA & 0 & LT & 25 & 77 & 55.1 & 32.5 \\
\hline LAO-8 & $\mathrm{L} A$ & 0 & LT & 25 & 77 & 30.5 & 18.0 \\
\hline$L 4 I-9$ & $L A$ & 1 & LT & 55 & 131 & 88.1 & 52.0 \\
\hline $\mathrm{L} 4 \mathrm{O}-4$ & LA & $\mathrm{O}$ & LT & 55 & 131 & 86.4 & 51.0 \\
\hline LAI -5 & LA & 1 & $L T$ & 70 & 158 & 105.1 & 62.0 \\
\hline
\end{tabular}

Table 5. Values of constants in Eq. 1, CTT, and USE for A212 Grade B material from shielded and irradiated walls of the Shippingport NST

\begin{tabular}{|c|c|c|c|c|c|c|c|c|c|}
\hline \multirow[b]{2}{*}{$\begin{array}{l}\text { Sample } \\
\text { Location }\end{array}$} & \multirow[b]{2}{*}{$\begin{array}{c}\text { Sample } \\
\text { Region }\end{array}$} & \multirow[b]{2}{*}{$\begin{array}{l}\text { Orient- } \\
\text { ation }\end{array}$} & \multicolumn{4}{|c|}{ Constants } & \multicolumn{2}{|c|}{ CTT $^{a}$} & \multirow[b]{2}{*}{$\begin{array}{c}\text { USE } \\
\left(\mathrm{J} / \mathrm{cm}^{2}[\mathrm{ft} \cdot \mathrm{lb}]\right)\end{array}$} \\
\hline & & & $\begin{array}{c}\mathrm{K}_{\mathrm{O}} \\
\left(\mathrm{J} / \mathrm{cm}^{2}\right)\end{array}$ & $\begin{array}{c}B \\
\left(\mathrm{~J} / \mathrm{cm}^{2}\right) \\
\end{array}$ & $\begin{array}{c}\mathrm{C} \\
\left.{ }^{\circ} \mathrm{C}\right) \\
\end{array}$ & $\begin{array}{c}\mathrm{D} \\
\left({ }^{\circ} \mathrm{C}\right) \\
\end{array}$ & $\begin{array}{l}20.4-\mathrm{J} \\
\left(\mathrm{C}\left({ }^{\circ} \mathrm{F}\right)\right. \\
\end{array}$ & $\begin{array}{c}41 \neg \mathrm{J} \\
\left({ }^{\circ} \mathrm{C}\left({ }^{\circ} \mathrm{F}\right)\right.\end{array}$ & \\
\hline \multicolumn{10}{|c|}{ Shielded Outer Wall } \\
\hline All & $\begin{array}{l}\text { Inner \& } \\
\text { Outer }\end{array}$ & $\mathrm{LT}$ & 8.6 & 46.7 & 33.5 & 23.2 & $16(61)$ & $31(88)$ & $102(60)$ \\
\hline 14.15 & Center & LT & 6.7 & 48.2 & 26.0 & 23.3 & $9(48)$ & $24(75)$ & $103(61)$ \\
\hline All & $\begin{array}{l}\text { Inner \& } \\
\text { Outer }\end{array}$ & $\mathrm{TL}$ & 8.0 & 29.3 & 33.5 & 30.3 & $20(68)$ & $49(120)$ & $67(40)$ \\
\hline 14.15 & Center & TL & $8.0^{b}$ & 33.5 & 18.4 & $30.3^{b}$ & $2(36)$ & $27(81)$ & $74(44)$ \\
\hline \multicolumn{10}{|c|}{ Irradiated Inner Wall } \\
\hline $2,8,3,9$ & Inner & LT & 8.3 & 33.6 & 53.3 & 17.2 & $44(111)$ & $58(136)$ & $76(45)$ \\
\hline $2,8,3,9$ & Outer & LT & 8.2 & 34.3 & 47.1 & 16.1 & $39(102)$ & $51(124)$ & 77 (45) \\
\hline $2,8,3,9,14,15$ & Allc & $T L$ & 4.1 & 24.1 & 46.1 & 25.5 & $43(109)$ & - & $52(31)$ \\
\hline
\end{tabular}

a Charpy transition temperature at the $20.3-\mathrm{J}(15 \mathrm{ft} \cdot \mathrm{lb})$ and $41 \mathrm{~J}(30 \mathrm{ft} \cdot \mathrm{lb})$ levels.

$b$ Values of $K_{0}$ and $D$ are assumed to be the same as those for inner/outer region.

c Inner- and outer-region specimens from Locations 2, 8, 3. and 9 and center region specimens from Locations 14 and 15.

and $2{ }^{\circ} \mathrm{C}\left(36^{\circ} \mathrm{F}\right)$ and $74 \mathrm{~J} / \mathrm{cm}^{2}(\approx 44 \mathrm{ft} \cdot \mathrm{lb})$ for the $\mathrm{TL}$ orientation. These variations are consistent with the throughwall variations in hardness. The hardness of the center region is in the range of $R_{B}$ 73-75 (132-142 DPH); for the :nner or outer regions it is 78-84 (144-162 $\mathrm{DPH})$.

Charpy-impact data for material from Location L4 of the shielded wall, shown in Fig. 10, are in good agreement with the data from other locations of the wall. The results indicate that the Charpy transition curves for the shielded wall of the NST represent baseline data for nonirradiated material. As discussed in Section 2, the hardness of the center of the wall varies significantly at different axial and azimuthal locations. The data for the center specimens may not be representative of all locations. Therefore, results from only the inner and outer regions of the wall are used to characterize irradiation embrittlement. 


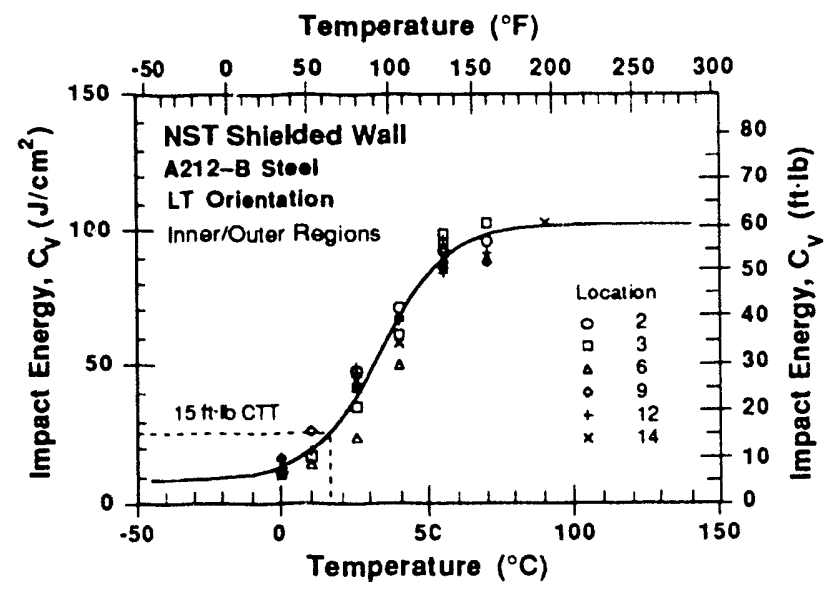

(a)

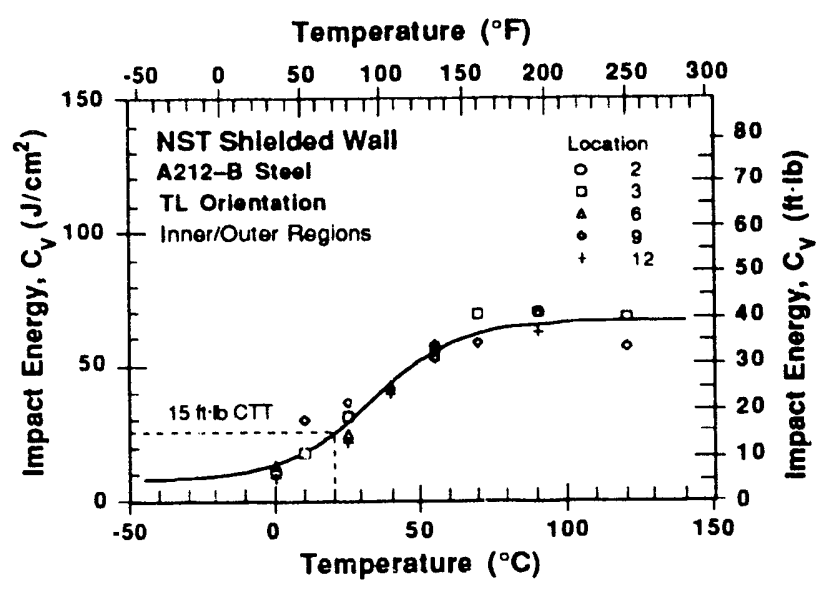

(b)

Figure 8. Best-fit Charpy transition curves for (a) LT and (b) TL specimens from the innerand outer-10-mm regions of the shielded wall of the Shippingport NST

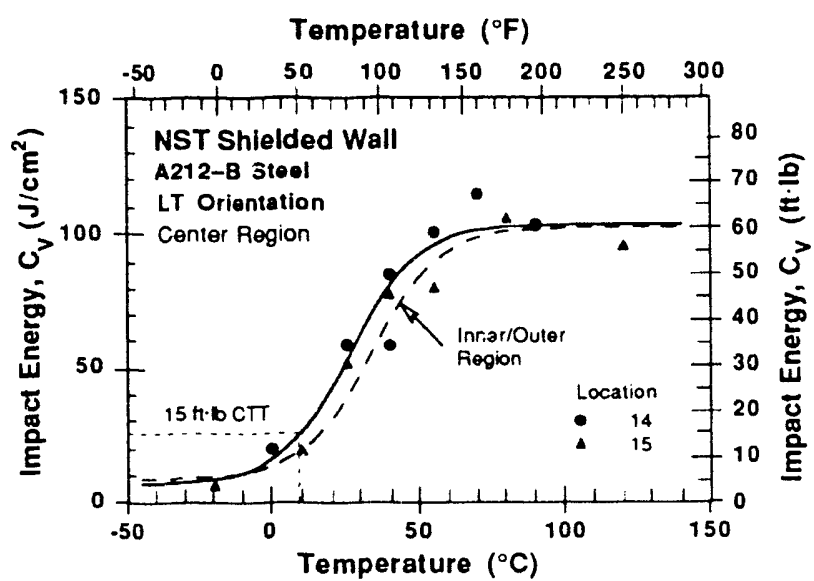

(a)

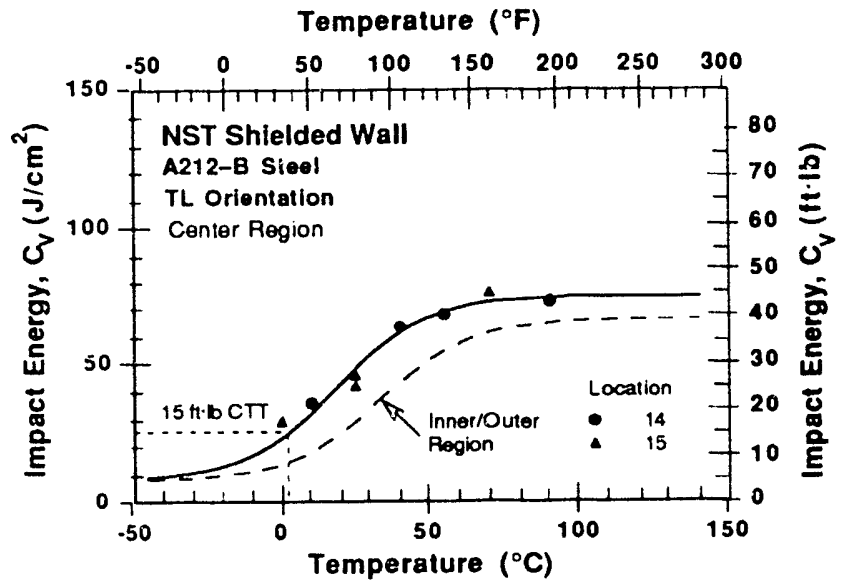

(b)

Figure 9. Best-fit Charpy transition curves for (a) LT and (b) TL specimens from the center$10-\mathrm{mm}$ region of the shielded wall of the Shippingport NST

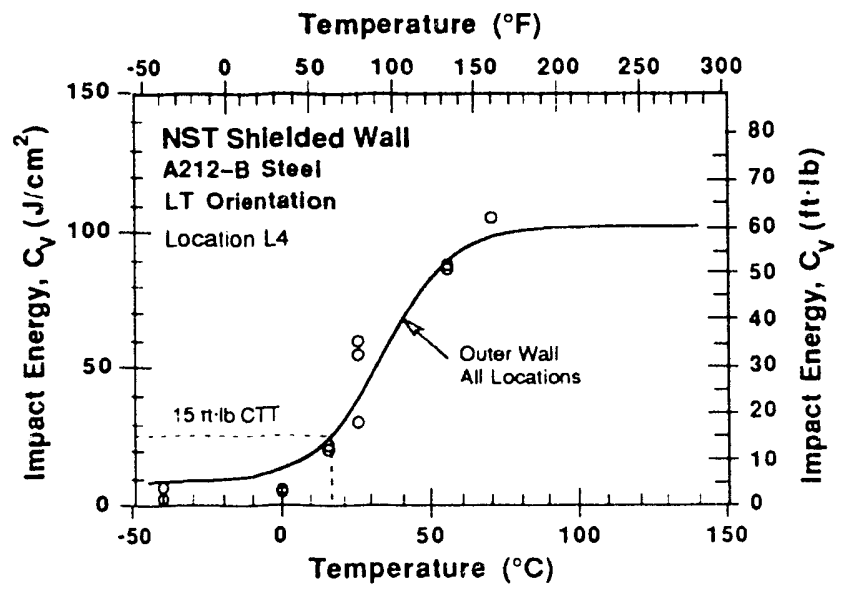

Figure 10.

Best-fit Charpy transition curve for $L T$ specimens from Location L4 of the shielded wall of the Shippingport NST 


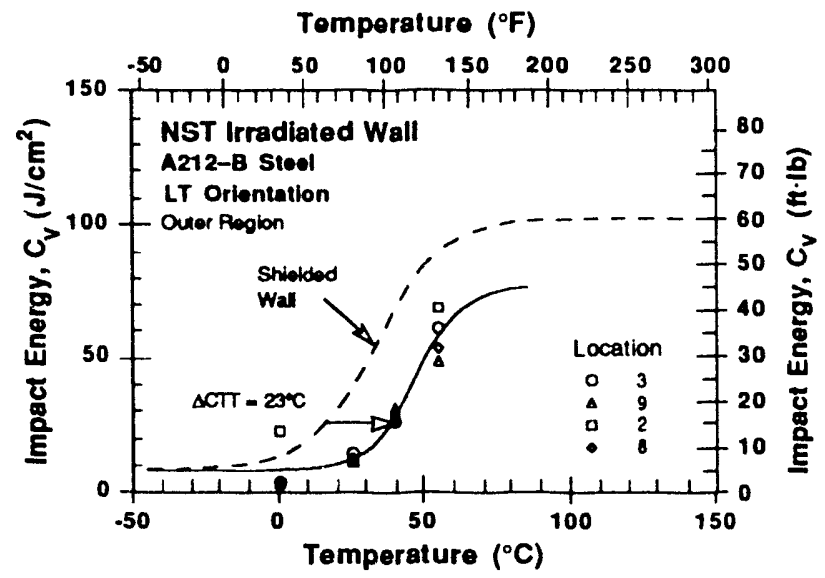

(a)

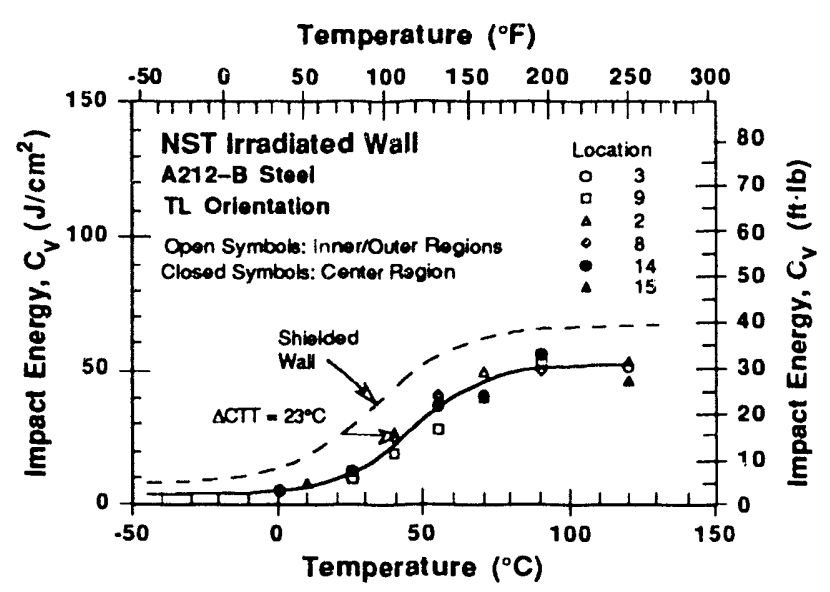

(c)

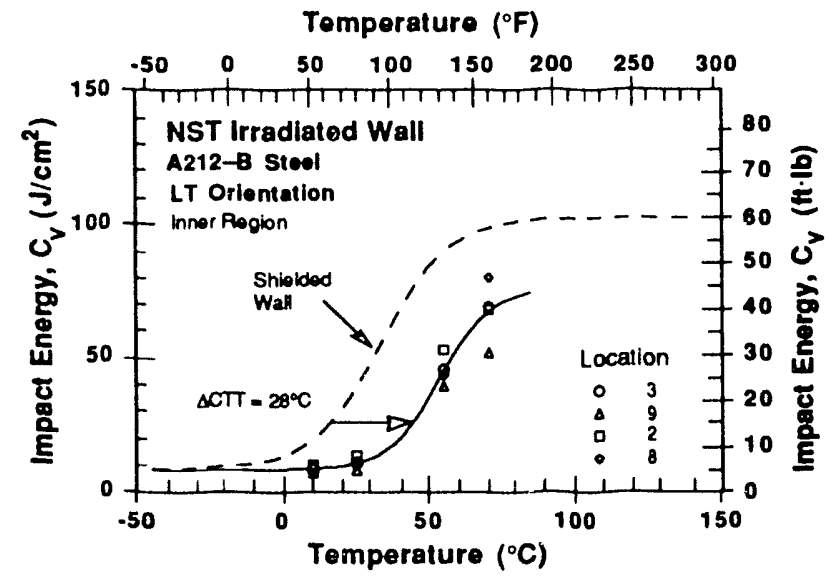

(b)

Figure 11.

Best-fit Chai py transition curves for (a) outerregion $L T$ specimens, (b) inner-region $L T$ specimens, and (c) TL specimens from all regions of the irradiated wall of the Shippingport NST

The transition curves for the LT and TL specimens from Locations 3, 9, 2, and 8 of the irradiated wall of the NST are shown in Fig. 11. The irradiated specimens show a higher CTT and lower USE relative to those from the essentially nonirradiated shielded wall. The effect of minor differences in total fluence between Locations 2 and $8\left(\approx 4 \times 10^{17} \mathrm{n} / \mathrm{cm}^{2}\right.$ fluence) and Locations 3 and $9\left(\approx 6 \times 10^{17} \mathrm{n} / \mathrm{cm}^{2}\right.$ fluence) are minimal and cannot be established from the data; Location 9 appears to have a slightly higher CTT than the other locations. Some effects of position through the thickness of the wall are observed for LT specimens, whose shift in CTT is greater for specimens from inner-region than for outerregion specimens. The values of CTT are $39^{\circ} \mathrm{C}\left(102^{\circ} \mathrm{F}\right)$ for the outer region and $44^{\circ} \mathrm{C}$ $\left(111^{\circ} \mathrm{F}\right)$ for the inner region, a shift of $\approx 23$ and $28^{\circ} \mathrm{C}\left(\approx 41\right.$ and $\left.50^{\circ} \mathrm{F}\right)$ for the outer and inner regions, respectively. The USE cannot be directly established from the data in Figs. 11 a and $11 \mathrm{~b}$. However, the specimens tested at $55^{\circ} \mathrm{C}\left(131^{\circ} \mathrm{F}\right)$ show $100 \%$ shear fracture; thus, the impact energy of these specimens, i.e., an average of $=77 \mathrm{~J} / \mathrm{cm}^{2}(45 \mathrm{ft} \cdot \mathrm{lb})$, is representative of USE.

The shift in CTT of the irradiated-wall TL specimens (Fig. $11 \mathrm{c})$ is also $23^{\circ} \mathrm{C}\left(41^{\circ} \mathrm{F}\right)$, similar to that for the LT specimens. However, the USE is lower, i.e., $52 \mathrm{~J} / \mathrm{cm}^{2}(31 \mathrm{ft} \cdot \mathrm{lb})$. The effect of position through the thickness of the wall is minimal for the these specimens. The impact energies for the center specimens are comparable to those for specimens from 
Table 6. Tensile test results for irrcdiated and shielded walls of the Shippingport NST

\begin{tabular}{|c|c|c|c|c|c|c|c|c|c|}
\hline \multirow[b]{2}{*}{$\begin{array}{c}\text { Specimen } \\
\text { Number }\end{array}$} & \multirow[b]{2}{*}{ Location } & \multirow[b]{2}{*}{ Region ${ }^{a}$} & \multirow[b]{2}{*}{ Fluence ${ }^{b}$} & \multirow[b]{2}{*}{$\begin{array}{c}\text { Test } \\
\text { Temp. } \\
\left({ }^{\circ} \mathrm{C}\right) \\
\end{array}$} & \multicolumn{2}{|c|}{ Engineering } & \multirow{2}{*}{$\begin{array}{c}\text { True } \\
\text { Fracture } \\
\text { Stress } \\
\text { (MPa) }\end{array}$} & \multirow[b]{2}{*}{$\begin{array}{c}\text { Elong- } \\
\text { ation } \\
(\%)\end{array}$} & \multirow[b]{2}{*}{$\begin{array}{c}\text { Red. in } \\
\text { Area } \\
(\%)\end{array}$} \\
\hline & & & & & $\begin{array}{c}0.2 \% \text { Yield } \\
\text { Stress } \\
\text { (MPa) }\end{array}$ & $\begin{array}{c}\text { Ultimate } \\
\text { Stress } \\
\text { (MPa) }\end{array}$ & & & \\
\hline \multicolumn{10}{|c|}{ Irradiated Inner Wall } \\
\hline I3RI & 3 & I & $6 \times 10^{17}$ & 25 & 368.0 & 572.1 & 777.3 & 28.2 & 43.9 \\
\hline 13RC & 3 & $\mathrm{C}$ & & 25 & 323.0 & 502.6 & 686.8 & 31.3 & 47.6 \\
\hline I3RO & 3 & $\mathrm{O}$ & & 25 & 3540 & 556.6 & 769.5 & 28.7 & 43.5 \\
\hline I2RI & 2 & 1 & $4 \times 10^{17}$ & 25 & 325.2 & 538.3 & 779.5 & 29.4 & 46.4 \\
\hline I2RC & 2 & $\mathrm{C}$ & & 25 & 319.7 & 540.4 & 733.7 & 29.2 & 42.9 \\
\hline I2RO & 2 & O & & 25 & 368.0 & 548.8 & 730.5 & 27.8 & 41.4 \\
\hline I9RI & 9 & I & $6 \times 10^{17}$ & 55 & 333.6 & 520.1 & 665.7 & 27.2 & 39.9 \\
\hline I9RC & 9 & C & & 55 & 305.2 & 479.2 & 666.9 & 28.3 & 45.7 \\
\hline I9RO & 9 & 0 & & 55 & - & 524.5 & 706.0 & 27.2 & 41.9 \\
\hline I8RI & 8 & 1 & $4 \times 10^{17}$ & 55 & 304.4 & 521.0 & 716.5 & 28.8 & 45.6 \\
\hline I8RC & 8 & $\mathrm{C}$ & & 55 & 310.4 & 515.0 & 732.6 & 29.0 & 45.3 \\
\hline I8RO & 8 & 0 & & 55 & 322.1 & 520.6 & 725.9 & 30.4 & 44.0 \\
\hline \multicolumn{10}{|c|}{ Shielded Outer Wall } \\
\hline O3RI & 3 & I & - & 25 & 282.9 & 536.5 & 740.8 & 30.2 & 43.6 \\
\hline O3RC & 3 & C & & 25 & 277.6 & 493.9 & 627.8 & 32.4 & 41.8 \\
\hline O3RO & 3 & $\mathrm{O}$ & & 25 & 324.6 & 540.4 & 709.2 & 29.4 & 43.3 \\
\hline O6RI & 6 & I & - & 25 & 294.0 & 528.1 & 691.6 & 31.1 & 40.4 \\
\hline OGRC & 6 & $\mathrm{C}$ & & 25 & 270.0 & 532.0 & 709.7 & 30.6 & 40.6 \\
\hline OGRO & 6 & 0 & & 25 & 307.3 & 5.27 .2 & 728.3 & 30.3 & 43.4 \\
\hline O12RI & 12 & $!$ & - & 25 & 329.6 & 530.5 & 768.1 & 27.5 & 45.6 \\
\hline O12RC & 12 & $\mathrm{C}$ & & 25 & 256.2 & 491.5 & 715.0 & 33.5 & 48.1 \\
\hline Ol2RO & 12 & 0 & & 25 & 293.6 & 511.0 & 735.4 & 29.4 & 46.8 \\
\hline O2RI & 2 & I & - & 55 & 280.2 & 519.5 & 711.2 & 30.3 & 44.7 \\
\hline O2RC & 2 & C & & 55 & 264.7 & 483.9 & 608.0 & 32.2 & 44.9 \\
\hline O2RO & 2 & 0 & & 55 & 286.2 & 520.7 & 701.4 & 29.8 & 42.4 \\
\hline O9RI & 9 & I & - & 55 & 248.2 & 489.9 & 669.5 & 31.3 & 46.2 \\
\hline O9RC & 9 & C & & 55 & 227.8 & 452.4 & 640.5 & 33.5 & 47.3 \\
\hline OARO & 9 & 0 & & 55 & 279.6 & 492.9 & 727.9 & 30.6 & 47.6 \\
\hline
\end{tabular}

a 1,0 , and $\mathrm{C}$ represent the inner-, outer-, and center-5-mm regions, respectively, across the wall thickness.

b Represent the values at the inner surface of the wall. Flivence for the outer-region samples is estimated to be a factor of 1.5 lower than that for inner-region samples.

the inner and outer region. Center specimens were obtained from locations 14 and 15 . where the variation in through-wall hardness was minimal

\subsubsection{Tensile Properties}

Tensile tests were conducted at room temperature and at $55^{\circ} \mathrm{C}\left(131^{\circ} \mathrm{F}\right)$ on $\mathrm{LT}$ specimens from several locations of the irradiated and shielded walls of the NST and from three regions across the thickness of the wall. The results of these tests, given in Table 6, indicate little or no variation with vertical and azimuthal position. The tensile strengths of the irradiated wall are higher than those for the shielded wall. The tensile properties also reflect the effect of variations in hardness observed across the thickness of the wall. 1.e., the yield and ultimate stresses for specimens fror. the center of the wall are always lower than 
those for specimens from inner or outer regions. However, the increases in yield and ultimate stress due to irradiation is independent of position through the thickness. The increases in yield and ultimate stress are, resper ively, $\approx 51$ and $20 \mathrm{MPa}(\approx 7.4$ and $2.9 \mathrm{ks} i)$ at room temperature, and $\approx 38$ and $18 \mathrm{MPa}(\approx 5.5$ and $2.6 \mathrm{ksi})$ at $55^{\circ} \mathrm{C}$.

The tensile properties of the NST material were also estimated from the Charpy-impact data. The yield stress is estimated from the expression

$$
\sigma_{\mathrm{y}}=\mathrm{AP} \mathrm{yB} / \mathrm{Wb}^{2} \text {. }
$$

taken from Ref. 8, where $\mathrm{Py}$ is the yield load obtained from the load-time traces of the instrumented Charpy tests, $W$ is the specimen width, $B$ is the specimen thickness, $b$ is the uncracked ligament, and $\mathrm{A}$ is a constant obtained by comparing the tensile and Charpy data for LT specimens tested at room temperature and at $55^{\circ} \mathrm{C}$. The best-fit value of $\mathrm{A}$ was 1.73 . Yield stresses estimated from Charpy-impact tests and those obtained from tensile tests for material from the irradiated and shielded walls are shown in Fig. 12. The results show the expected decrease in yield stress with an increase in test temperature. Irradiation increases the yield stress at all test temperatures.

The tensile data for the Shippingport NST show very good agreement with the correlations between the increases in CTT and yield stress that have been developed for pressure vessel steel. 9.10 The CTT shift (in ${ }^{\circ} \mathrm{C}$ ) and increase in yield stress $\Delta \sigma_{\mathrm{y}}$ (in $\mathrm{MPa}$ ) is expressed as

$$
\Delta \mathrm{CTT}=\mathrm{C} \Delta \sigma_{\mathrm{y}},
$$

where $\mathrm{C}$ is $\approx 0.5^{\circ} \mathrm{C} / \mathrm{MPa}$ for plate material, and $0.65^{\circ} \mathrm{C} / \mathrm{MPa}$ for welds. The shift in the CTT of both LT and TL specimens is $23^{\circ} \mathrm{C}\left(28^{\circ} \mathrm{C}\right.$ for LT specimens from inner regions), and the increase in yield stress is $51 \mathrm{MPa}$.

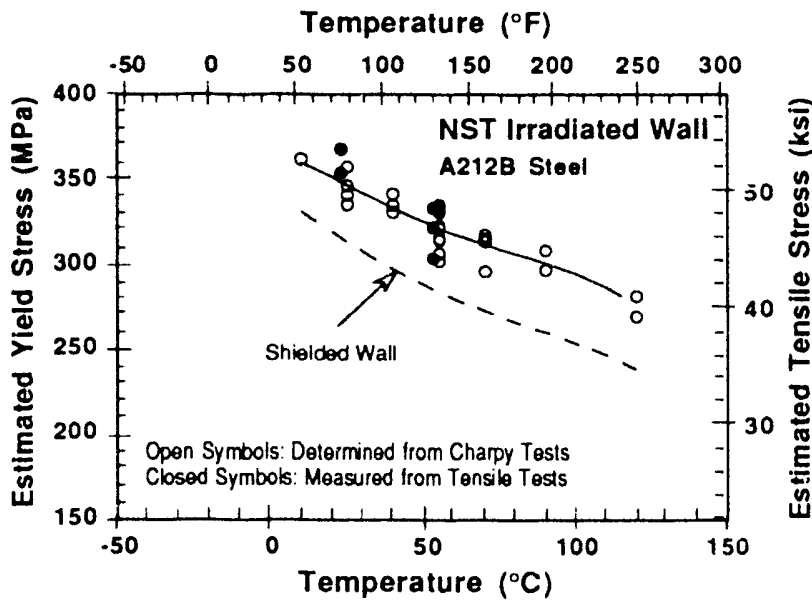

(a)

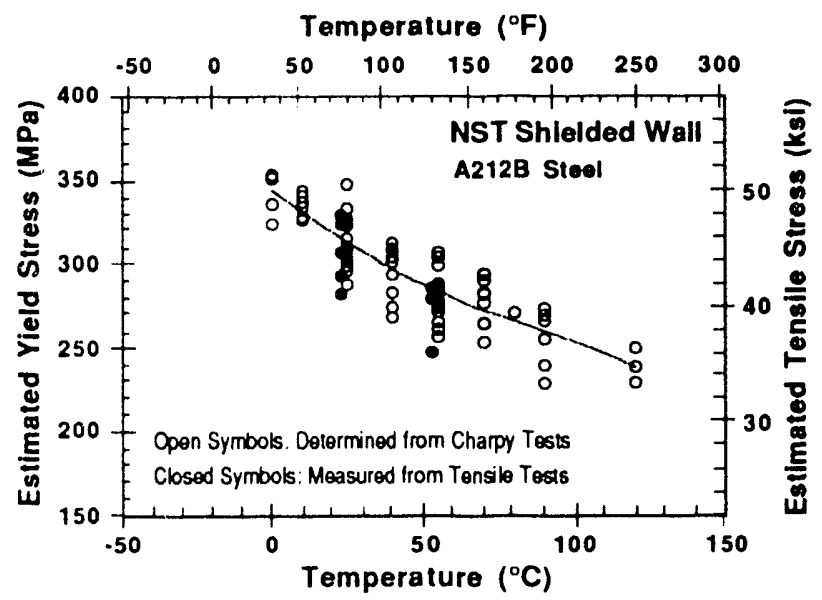

(b)

Figure 12. Yield stress estimated from Charpy-impact tests and measured from tensile tests for (a) irradiated and (b) shielded walls of the Shippingport NST 


\subsubsection{Recovery Annealing}

Annealing studies were conducted to examine the recovery behavior of the embrittled material. Samples from Locations 3, 9, and 8 of the irradiated wall and locations 3 and 6 of the shielded wall were annealed up to $154 \mathrm{~h}$ at $400^{\circ} \mathrm{C}\left(752^{\circ} \mathrm{F}\right)$ and recovery behavior was characterized by hardness measurements. As mentioned in Section 2, hardness changes across the thickness of the NST wall. Consequently, hardness measurements were made on surfaces that were normal to the hardness profile, i.e., sections marked A, B, C, and D in Figs. 4-6. Hardness values are approximately the same along these sections, although they may vary significantly among the sections. The recovery behavior was established by hardness measurements on the same specimen before and after annealing for various times. The results are given in Table 7.

The results indicate that hardness of the irradiated inner wall decreases whereas that of the shielded outer wall increases after annealing. The changes in hardness occur within $1 \mathrm{~h}$ at $400^{\circ} \mathrm{C}$ and there is little or no change in hardness after annealing for longer times. The results for the shielded outer wall, shown in Fig. 13, indicate that the increase in hardness is observed along sections B, C, and D, whereas, section A shows little or no change in hardness The average increase in hardness for Locations 3 and 6 along sections $B, C$, and $D$ is $R_{B} 1.4$ (DPH 4.4). It can be argued that the observed hardness profiles (Figs. 4-6) are a result of residual stresses, 1.e., tensile stresses in the center and compressive stresses near the surface of the wall. Studies on the effect of residual stresses on hardness indicate that hardness measurements change when a uniaxial stress is applied perpendicular to the load of the hardness tester; hardness decreases linearly with applied tensile stress, whereas compressive stress causes little or no change in hardness. 11.12 However, annealing studies were conducted on 10-mm-wide samples from the inner and outer regions of the wall. Therefore, sections A, B, C, and D should have compressive stresses and little or no effect on hardness measurements. Furthermore, hardness values for all specimens prior to aunealing are in very good agreement with the results in Figs. 4-6. The observed hardness profiles are likely caused by metallurgical factors. The increase in hardness after annealing may be attributed to thermal aging.

The changes in hardness of material from the irradiated inner wall, shown in Fig. 14, reflect the differences in fluence and flux levels of the different locations, 1.e., the decrease for Locations 3 and $9\left(\approx 6 \times 10^{17} \mathrm{n} / \mathrm{cm}^{2}\right.$ fluence) is greater than for Location $8\left(\approx 4 \times 10^{17}\right.$

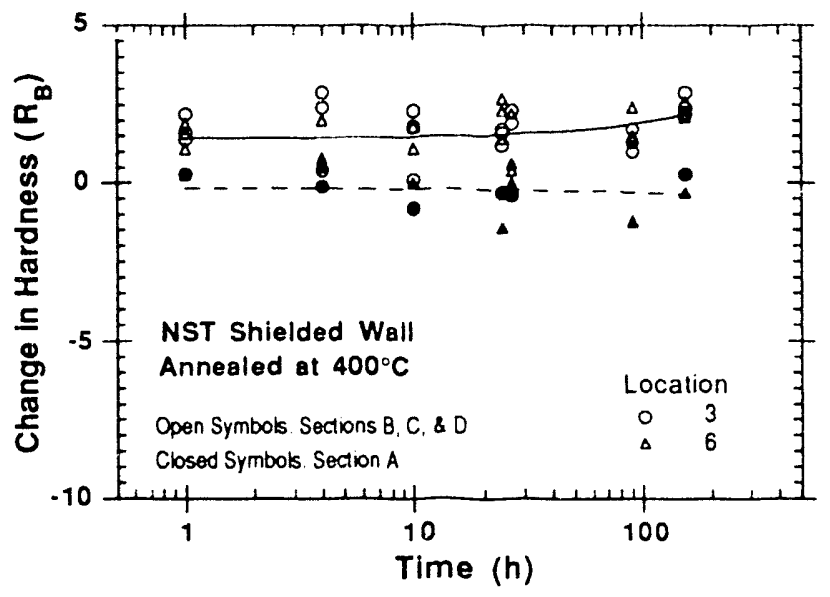

Figure 13.

Change in hardness of the shielded wall after annealing at $400^{\circ} \mathrm{C}$ 
Table 7. Hardness values (Rockwell B)a of the Shippingport NST material from irradiated and shielded walls after annealing at $400^{\circ} \mathrm{C}$

\begin{tabular}{|c|c|c|c|c|c|c|c|c|c|}
\hline \multirow[b]{2}{*}{ Location } & \multirow[b]{2}{*}{ Section $^{b}$} & \multicolumn{8}{|c|}{ Annealing Time (h) } \\
\hline & & 0 & 1 & 4 & 10 & 24 & 26.5 & 90 & 154 \\
\hline & \multicolumn{9}{|c|}{ Shielded Outer Wall } \\
\hline \multirow[t]{8}{*}{3} & A & 82.5 & $82 . \overline{8}$ & 82.4 & 81.7 & & 82.2 & & \\
\hline & B & 76.8 & 78.4 & 79.2 & 78.6 & & 78.7 & & \\
\hline & $\mathrm{C}$ & 75.8 & 78.0 & 78.7 & 78.1 & & 78.1 & & \\
\hline & $\mathrm{D}$ & 83.6 & 85.0 & 84.0 & 83.7 & & 83.2 & & \\
\hline & A & 82.6 & & & & 82.3 & & 84.3 & 82.9 \\
\hline & B & 76.6 & & & & 78.3 & & 77.6 & 79.0 \\
\hline & $\mathrm{C}$ & 76.3 & & & & 77.5 & & 78.0 & 79.2 \\
\hline & $\mathrm{D}$ & 82.0 & & & & 83.6 & & 83.3 & 84.2 \\
\hline \multirow[t]{9}{*}{6} & A & 83.6 & 83.9 & 84.2 & 83.6 & & 83.6 & & \\
\hline & B & 77.9 & 79.8 & 79.9 & 79.7 & & 80.1 & & \\
\hline & $\mathrm{C}$ & 79.4 & 80.5 & 79.9 & 79.5 & & 79.8 & & \\
\hline & $\mathrm{D}$ & 81.2 & 82.8 & 82.0 & 82.3 & & 81.8 & & \\
\hline & A & 84.9 & & & & 83.5 & & 83.7 & 84.6 \\
\hline & B & 77.7 & & & & 80.0 & & 80.1 & 80.3 \\
\hline & $\mathrm{C}$ & 78.5 & & & & 79.9 & & 80.0 & 80.7 \\
\hline & $D$ & 81.2 & & & & 83.9 & & 82.6 & 83.3 \\
\hline & \multicolumn{9}{|c|}{ Irradiated Inner Wall } \\
\hline 3 & A & 87.5 & 83.2 & 83.5 & 83.6 & & 82.8 & & \\
\hline & B & 80.7 & 77.1 & 76.8 & 77.5 & & 76.8 & & \\
\hline & $\mathrm{C}$ & 81.7 & 77.8 & 78.0 & 76.9 & & 77.2 & & \\
\hline & $\mathrm{D}$ & 84.9 & 83.2 & 82.8 & 82.6 & & 82.6 & & \\
\hline & A & 87.3 & & & & 83.8 & & 84.1 & 84.6 \\
\hline & $\mathrm{B}$ & 80.9 & & & & 77.2 & & 77.1 & 77.8 \\
\hline & $\mathrm{C}$ & 80.4 & & & & 75.2 & & 75.5 & 76.1 \\
\hline & D & 86.8 & & & & 85.2 & & 85.1 & 85.6 \\
\hline \multirow[t]{8}{*}{9} & A & 86.8 & 82.5 & 82.6 & 83.3 & & 82.6 & & \\
\hline & B & 82.3 & 78.4 & 78.1 & 78.2 & & 78.2 & & \\
\hline & C & 81.6 & 76.2 & 76.8 & 77.1 & & 75.9 & & \\
\hline & D & 84.5 & 82.6 & 82.0 & 79.6 & & 80.9 & & \\
\hline & A & 87.9 & & & & 82.6 & & 83.8 & 83.4 \\
\hline & B & 82.1 & & & & 77.7 & & 77.9 & 78.6 \\
\hline & C & 81.9 & & & & 76.3 & & 76.4 & 77.6 \\
\hline & D & 84.2 & & & & 81.1 & & 81.6 & 82.2 \\
\hline \multirow[t]{8}{*}{8} & A & 84.8 & 81.8 & 81.4 & 81.6 & & 81.3 & & \\
\hline & B & 84.6 & $R^{n} 2$ & 82.4 & 80.4 & & 81.5 & & \\
\hline & $\mathrm{C}$ & 86.0 & 83.0 & 82.8 & 83.1 & & 83.1 & & \\
\hline & $D$ & 83.3 & 81.5 & 80.8 & 81.1 & & 80.4 & & \\
\hline & A & 86.6 & & & & 84.3 & & 82.7 & 83.3 \\
\hline & $\mathrm{B}$ & 81.7 & & & & 78.5 & & 78.3 & 78.4 \\
\hline & $\mathrm{C}$ & 83.2 & & & & 79.1 & & 79.5 & 80.7 \\
\hline & $\mathrm{D}$ & 83.4 & & & & 81.3 & & 81.6 & 81.4 \\
\hline
\end{tabular}

$\mathrm{n} / \mathrm{cm}^{2}$ fluence). Also, the decrease in hardness along Sections $\mathrm{A}, \mathrm{B}$, and $\mathrm{C}$ is greater than that along Section D. As mentioned earlier, total fluence decreases by a factor of 2-3 across the thickness of the irradiated inner wall. The decrease in hardness for Locations 3, 9, and 8 is respectively, $R_{B} 4.1,4.6$, and 3.1 (DFH 12.4, 14.0. and 9.2) along Sections $A, B$, and C, and $R_{B} 2.1,2.8$, and 2.3 (DPH 6.4, 8.4, and 7.0) along Section $D$. Contrúl samples from the 
shielded outer wall show an increase in hardness after annealing. Consequently, actual values of irradiation hardening for Locations 3 , 9, and 8 may be higher by $R_{B} 1.4$ (DPH 4.4) than the change in hardness shown in Fig. 14.

Correlations between increases in hardness and yield stress from radiation hardening of pressure vessel steels indicate that the increase in yield stress (in MPail is 3.5 times the increase in hardness (in DPH) $.9,10$ Based on tensile and Charpy-impact data, the increase in hardness for the Shippingport NST should be $\approx 15 \mathrm{DPH}$. The measured values for Locations 3, 9, and 8 are in falr agreement with the estimation.

Charpy-impact data for TL specimens from the irradiated and shielded walls of the NST, annealed for $2 \mathrm{~h}$ at $400^{\circ} \mathrm{C}$, are given in Table 8; Charpy transition curves are shown in Fig. 15. The results indicate complete recovery from radiation-induced embrittlement, i.e.,

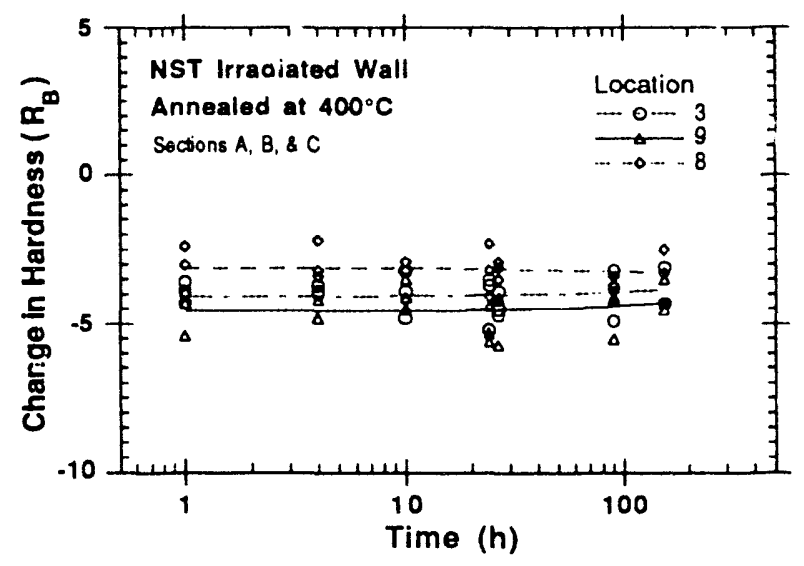

(a)

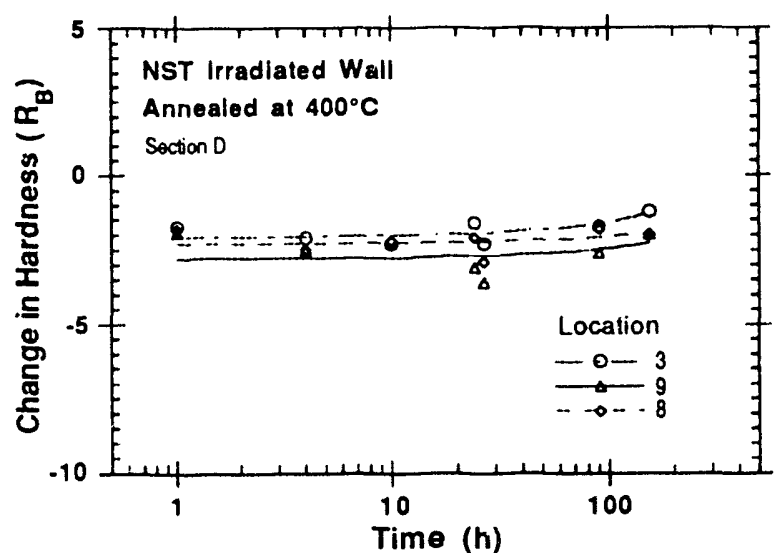

(b)

Figure 14. Change in hardness measured on (a) Sections $A, B$, and $C$ and (b) Section $D$ of the irradiated wall after annealing at $400^{\circ} \mathrm{C}$

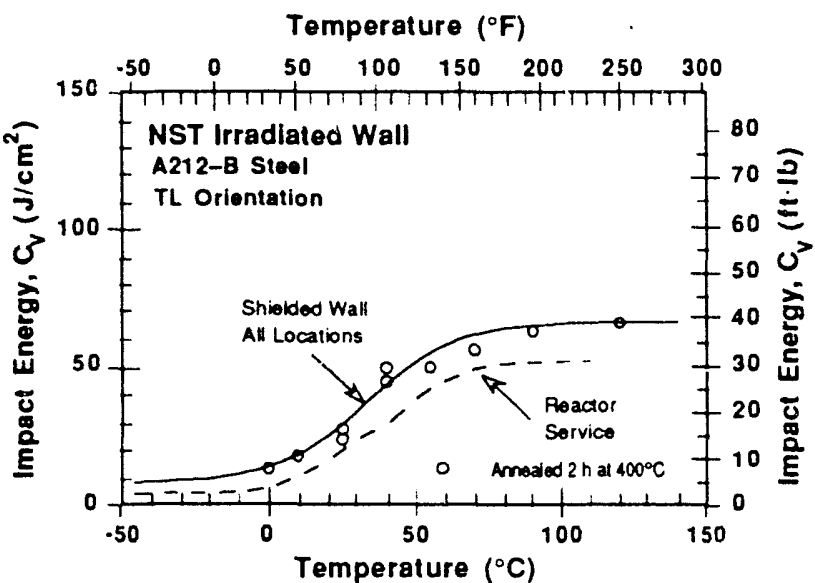

(a)

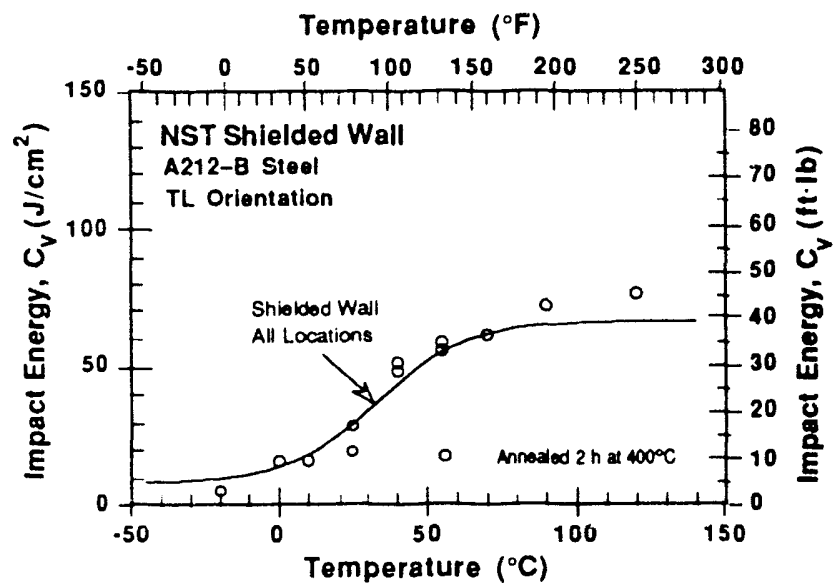

(b)

Figure 15. Charpy transition curves for annealed TL specimens from (a) the irradiated and (b) the shielded wall of the Shippingport NST 
Table 8. Charpy-impact data for material from the irradiated and shielded walls of the Shippingport NST after annealing at $400^{\circ} \mathrm{C}$

\begin{tabular}{|c|c|c|c|c|c|c|c|c|c|}
\hline \multirow{2}{*}{$\begin{array}{c}\text { Specimen } \\
\text { ID }\end{array}$} & \multirow[b]{2}{*}{ Location } & \multirow[b]{2}{*}{ Regiona } & \multirow{2}{*}{$\begin{array}{l}\text { Orien- } \\
\text { tation }\end{array}$} & \multicolumn{2}{|c|}{ Test Temp. } & \multicolumn{2}{|c|}{ Impact Energy } & \multicolumn{2}{|c|}{ Load $(\mathrm{kN})$} \\
\hline & & & & $\left({ }^{\circ} \mathrm{C}\right)$ & $\left({ }^{\circ} \mathrm{F}\right)$ & $\left(\mathrm{J} / \mathrm{cm}^{2}\right)$ & $(\mathrm{ft} \cdot \mathrm{lb})$ & Yield & Maximum \\
\hline \multicolumn{10}{|c|}{ Irradiated Inner Wall } \\
\hline $\begin{array}{l}103 T-05 A \\
\text { I03T-06A }\end{array}$ & $\begin{array}{l}3 \\
3\end{array}$ & $\begin{array}{l}\text { I } \\
\text { O }\end{array}$ & $\begin{array}{l}\mathrm{TL} \\
\mathrm{TL}\end{array}$ & $\begin{array}{r}25 \\
0\end{array}$ & $\begin{array}{l}77 \\
32\end{array}$ & $\begin{array}{l}27.7 \\
13.0\end{array}$ & $\begin{array}{r}16.3 \\
7.7\end{array}$ & $\begin{array}{l}12.206 \\
11.083\end{array}$ & $\begin{array}{l}12.206 \\
11.083\end{array}$ \\
\hline $\begin{array}{l}109 T-05 A \\
109 T-06 A\end{array}$ & $\begin{array}{l}9 \\
9\end{array}$ & $\begin{array}{l}1 \\
0\end{array}$ & $\begin{array}{l}\text { TL } \\
\text { TL }\end{array}$ & $\begin{array}{l}55 \\
40\end{array}$ & $\begin{array}{l}131 \\
104\end{array}$ & $\begin{array}{l}50.0 \\
49.7\end{array}$ & $\begin{array}{l}29.5 \\
29.3\end{array}$ & $\begin{array}{l}11.278 \\
11.063\end{array}$ & $\begin{array}{l}12.167 \\
11.864\end{array}$ \\
\hline $\begin{array}{l}102 T-05 A \\
102 T-06 A\end{array}$ & $\begin{array}{l}2 \\
2\end{array}$ & $\begin{array}{l}1 \\
0\end{array}$ & $\begin{array}{l}T L \\
T L\end{array}$ & $\begin{array}{l}70 \\
10\end{array}$ & $\begin{array}{r}158 \\
50\end{array}$ & $\begin{array}{l}56.3 \\
18.0\end{array}$ & $\begin{array}{l}33.2 \\
10.6\end{array}$ & $\begin{array}{l}10.155 \\
10.194\end{array}$ & $\begin{array}{l}11.229 \\
10.194\end{array}$ \\
\hline $\begin{array}{l}108 \mathrm{~T}-05 \mathrm{~A} \\
108 \mathrm{~T}-06 \mathrm{~A}\end{array}$ & $\begin{array}{l}8 \\
8\end{array}$ & $\begin{array}{l}\mathrm{I} \\
\mathrm{O}\end{array}$ & $\begin{array}{l}T L \\
T L\end{array}$ & $\begin{array}{l}25 \\
40\end{array}$ & $\begin{array}{r}77 \\
104\end{array}$ & $\begin{array}{l}24.0 \\
44.7\end{array}$ & $\begin{array}{l}14.2 \\
26.4\end{array}$ & $\begin{array}{l}12.538 \\
11.219\end{array}$ & $\begin{array}{l}12.538 \\
12.245\end{array}$ \\
\hline $114 \mathrm{~T}-01 \mathrm{~A}$ & 14 & I & TL & 120 & 248 & 66.2 & 39.1 & 9.354 & 11.766 \\
\hline \multicolumn{9}{|c|}{ Shielded Outer Wall } & 11.805 \\
\hline OО2T-07A & 2 & I & $T L$ & 25 & 77 & 28.5 & 16.8 & 12.401 & 12.401 \\
\hline $002 T-05 A$ & 2 & I & TL & 55 & 131 & 55.8 & 33.0 & 10.956 & 12.459 \\
\hline O02T-08A & 2 & 0 & $T L$ & 0 & 32 & 15.7 & 9.3 & 11.014 & 11.014 \\
\hline O02T-06A & 2 & 0 & $\mathrm{TL}$ & 90 & 194 & 72.1 & 42.5 & 10.448 & 12.596 \\
\hline O03T-05A & 3 & 1 & TL & 10 & 50 & 16.0 & 9.4 & 10.800 & 10.800 \\
\hline O03T-07A & 3 & 1 & TL & 40 & 104 & 48.5 & 28.6 & 11.171 & 12.44 \\
\hline O03T-08A & 3 & O & TL & 120 & 248 & 76.6 & 45.2 & 9.647 & 12.381 \\
\hline O06T-07A & 6 & 1 & TL & 25 & 77 & 19.3 & 11.4 & 11.473 & 11.473 \\
\hline O06T-06A & 6 & 0 & TL & -20 & -4 & 5.1 & 3.0 & 9.667 & 9.667 \\
\hline 006T-08A & 6 & 0 & TL & 55 & 131 & 58.7 & 34.6 & 11.024 & 12.684 \\
\hline $012 \mathrm{~T}-07 \mathrm{~A}$ & 12 & I & TL & 40 & 104 & 51.6 & 30.4 & 10.546 & 11.678 \\
\hline O12T-08A & 12 & 0 & $T L$ & 70 & 158 & 61.5 & 36.3 & 9.852 & 11.483 \\
\hline
\end{tabular}

a $I$ and $O$ represent the inner- and outer-10-mm regions, respectively, across the wall thickness.

the transition curve of the annealed specimens from the irradiated wall is identical to that of the shielded wall. Annealing has little or no effect on the transition curve of the shielded wall. These results confirm that the data for material from the shielded outer wall represents baseline data for nonirradiated material.

\subsection{Weld Metal}

Weld samples were obtained from Location 1 on the shielded wall and three positions on the irradiated wall (Locations 14 and 15 with $\approx 3 \times 10^{17} \mathrm{n} / \mathrm{cm}^{2}$ fluence and Location 13 with $\approx 2 \times 10^{17} \mathrm{n} / \mathrm{cm}^{2}$ fluence). All of the welds were transverse to the plate rolling direction. Charpy-impact test specimens were machined perpendicular to the weld from the inner and outer regions of the wall. The elemental compositions of weld metals from different locations are given in Table 1; only minor variations in silicon and copper content were observed. Charpy data for the shielded- and irradiated-wall welds are given in Tables 9 and 10 , respectively.

Annealing studies were also conducted on weld metal specimens to obtain baseline data and help characterize the irradiation embrittlement of the welds. Charpy data for annealed weld metal specimens from Location 1 of the shielded wall and Locations 14 and 15 of the irradiated 'vall aie also given in Tables 9 and 10. The results indicate that the Charpy transition curves for the annealed specimens do not always represent the baseline impact prop- 
Table 9. Charpy impact data for the shielded outer-wall weld of the Shippingport NST

\begin{tabular}{|c|c|c|c|c|c|c|c|c|c|}
\hline \multirow{2}{*}{$\begin{array}{c}\text { Specimen } \\
\text { II }\end{array}$} & \multirow[b]{2}{*}{ Location } & \multirow[b]{2}{*}{ Region ${ }^{a}$} & \multirow{2}{*}{$\begin{array}{l}\text { Orien- } \\
\text { tation }\end{array}$} & \multicolumn{2}{|c|}{ Test Temp. } & \multicolumn{2}{|c|}{ Impact Energy } & \multicolumn{2}{|c|}{ Load $(\mathbf{k N})$} \\
\hline & & & & $\left({ }^{\circ} \mathrm{C}\right)$ & $\left({ }^{\circ} \mathrm{F}\right)$ & $\left(\mathrm{H} / \mathrm{cm}^{2}\right)$ & $(\mathrm{ft} \cdot \mathrm{lb})$ & Yieid & Maximum \\
\hline \multicolumn{10}{|c|}{ Reactor Service } \\
\hline OOIR-03W & 1 & I & LT & $\overline{0}$ & 32 & 55.6 & 32.8 & 12.333 & 13.163 \\
\hline OOIR-11W & 1 & I & LT & 20 & 68 & 95.8 & 56.5 & 12.821 & 15.028 \\
\hline OOIR-05W & 1 & 1 & LT & 40 & 104 & 157.8 & 93.1 & 13.319 & 16.092 \\
\hline OOIR-07W & 1 & I & LT & 70 & 158 & 172.1 & 101.5 & 13.465 & 16.482 \\
\hline OOIR-09W & 1 & I & LT & 120 & 248 & 165.8 & 97.8 & 12.879 & 15.379 \\
\hline $001 R-12 W$ & 1 & 0 & $\mathrm{LT}$ & -40 & -40 & 29.2 & 17.2 & 15.526 & 16.082 \\
\hline OOIR-02W & 1 & 0 & LT & 10 & 50 & 81.5 & 48.1 & 13.797 & 16.209 \\
\hline COIR-04W & 1 & 0 & LT & 25 & 77 & 123.8 & 73.0 & 13.621 & 16.456 \\
\hline OOIR-06W & 1 & 0 & LT & 55 & 131 & 194.4 & 114.7 & 13.309 & 17.02 \\
\hline OOIR-C8W & 1 & 0 & LT & 90 & 194 & 190.4 & 112.3 & 12.557 & 16.121 \\
\hline \multicolumn{10}{|c|}{ Annealed } \\
\hline OOIR-13WA & 1 & I & LT & -20 & -4 & 45.9 & 27.1 & 14.53 & 16.775 \\
\hline OOIR-15WA & $i$ & I & LT & 10 & 50 & 104.6 & 61.7 & 13.944 & 17.264 \\
\hline OOIR-17WA & $i$ & I & LT & 40 & 104 & 151.8 & 89.6 & 13.465 & 16.492 \\
\hline OOIR-14WA & $i$ & 0 & LT & c & 32 & 150.2 & 88.6 & 14.051 & 17.547 \\
\hline OOIR-I6WA & $i$ & 0 & LT & 25 & 77 & 255.0 & 150.5 & 12.518 & 17.791 \\
\hline OOIR-18WA & $i$ & 0 & LT & 55 & 131 & 254.2 & 150.0 & 13.182 & 17.312 \\
\hline
\end{tabular}

a $I$ and $O$ represent the inner-and outer-10-mm regions, respectively, across the wall. thickness.

Table 10. Charpy impact data for the irradiated inner-wall weld of the Shippingport NST

\begin{tabular}{|c|c|c|c|c|c|c|c|c|c|c|}
\hline \multirow{2}{*}{$\begin{array}{c}\text { Specimen } \\
\text { ID }\end{array}$} & \multirow[t]{2}{*}{ Location } & \multirow[t]{2}{*}{ Region $^{a}$} & \multirow{2}{*}{$\begin{array}{c}\text { Fluence }^{b} \\
\left(\mathrm{n} / \mathrm{cm}^{2}\right)\end{array}$} & \multirow{2}{*}{$\begin{array}{l}\text { Orien- } \\
\text { tation }\end{array}$} & \multicolumn{2}{|c|}{ Test Temp. } & \multicolumn{2}{|c|}{ Impact Energy } & \multicolumn{2}{|c|}{ Load $(\mathrm{kN})$} \\
\hline & & & & & $\left.{ }^{\circ} \mathrm{C}\right)$ & $\left({ }^{\circ} \mathrm{F}\right)$ & $\left(\mathrm{J} / \mathrm{cm}^{2}\right)$ & (ft.lb) & Yield & Maximum \\
\hline $\begin{array}{l}\text { I13R-07W } \\
\text { I13R-01W } \\
\text { I13R-03W } \\
113 R-05 W \\
\text { I13R-08W } \\
113 R-02 W \\
\text { I13R-04W } \\
\text { I13R-06W }\end{array}$ & $\begin{array}{l}13 \\
13 \\
13 \\
13 \\
13 \\
13 \\
13 \\
13\end{array}$ & $\begin{array}{l}1 \\
1 \\
1 \\
1 \\
0 \\
0 \\
0 \\
0\end{array}$ & $2 \times 10^{17}$ & $\begin{array}{l}\text { LT } \\
\text { LT } \\
\text { LT } \\
\text { LT } \\
\text { LT } \\
\text { LT } \\
\text { LT } \\
\text { LT }\end{array}$ & $\begin{array}{r}-20 \\
10 \\
40 \\
70 \\
10 \\
25 \\
55 \\
90\end{array}$ & $\begin{array}{r}-4 \\
50 \\
104 \\
158 \\
50 \\
77 \\
131 \\
194\end{array}$ & $\begin{array}{r}19.8 \\
51.6 \\
134.7 \\
178.3 \\
136.5 \\
122.7 \\
174.6 \\
188.4\end{array}$ & $\begin{array}{r}11.7 \\
30.4 \\
79.5 \\
105.4 \\
80.5 \\
72.4 \\
103.0 \\
111.2\end{array}$ & $\begin{array}{l}16.502 \\
15.844 \\
15.311 \\
14.451 \\
15.428 \\
15.018 \\
14.647 \\
13.68\end{array}$ & $\begin{array}{l}16.502 \\
18.669 \\
18.084 \\
17.312 \\
18.572 \\
18.396 \\
17.928 \\
17.107\end{array}$ \\
\hline $\begin{array}{l}114 R-07 W \\
114 R-01 W \\
114 R-09 W \\
114 R-05 W \\
114 R-11 W \\
114 R-13 W \\
114 R-03 W \\
114 R-08 W \\
114 R-10 W \\
114 R-06 W \\
114 R-02 W \\
114 R-12 W \\
114 R-04 W\end{array}$ & $\begin{array}{l}14 \\
14 \\
14 \\
14 \\
14 \\
14 \\
14 \\
14 \\
14 \\
14 \\
14 \\
14 \\
14\end{array}$ & $\begin{array}{l}1 \\
1 \\
1 \\
1 \\
1 \\
1 \\
1 \\
0 \\
0 \\
0 \\
0 \\
0 \\
0\end{array}$ & $3 \times 10^{17}$ & $\begin{array}{l}\text { LT } \\
\text { LT } \\
\text { LT } \\
\text { LT } \\
\text { LT } \\
\text { LT } \\
\text { LT } \\
\text { LT } \\
\text { LT } \\
\text { LT } \\
\text { LT } \\
\text { LT } \\
\text { LT }\end{array}$ & $\begin{array}{r}-40 \\
0 \\
25 \\
40 \\
55 \\
70 \\
90 \\
-10 \\
10 \\
20 \\
40 \\
40 \\
70\end{array}$ & $\begin{array}{r}-40 \\
32 \\
77 \\
104 \\
131 \\
158 \\
194 \\
14 \\
50 \\
68 \\
104 \\
104 \\
158\end{array}$ & $\begin{array}{r}14.1 \\
38.0 \\
92.6 \\
66.2 \\
132.8 \\
140.3 \\
153.5 \\
63.3 \\
90.6 \\
95.4 \\
185.9 \\
150.8 \\
176.3\end{array}$ & $\begin{array}{r}8.3 \\
22.4 \\
54.6 \\
39.1 \\
78.4 \\
82.8 \\
90.6 \\
37.4 \\
53.5 \\
56.3 \\
109.7 \\
89.0 \\
104.0\end{array}$ & $\begin{array}{l}17.81 \\
16.6 \\
16.629 \\
15.652 \\
16.014 \\
15.233 \\
14.432 \\
15.662 \\
15.818 \\
15.711 \\
14.783 \\
15.428 \\
13.817\end{array}$ & $\begin{array}{l}17.81 \\
18.035 \\
19.666 \\
17.81 \\
18.914 \\
18.2 \mathrm{i} 1 \\
16.58 \\
18.543 \\
19.021 \\
17.859 \\
17.762 \\
16.601 \\
17.352\end{array}$ \\
\hline $\begin{array}{l}115 R-07 W \\
115 R-01 W \\
115 R-03 W \\
115 R-05 W \\
115 R-08 W \\
115 R-02 W \\
115 R-06 W \\
\text { I15R-04W }\end{array}$ & $\begin{array}{l}15 \\
15 \\
15 \\
15 \\
15 \\
15 \\
15 \\
15\end{array}$ & $\begin{array}{l}1 \\
1 \\
1 \\
1 \\
0 \\
0 \\
0 \\
0\end{array}$ & $3 \times 10^{17}$ & $\begin{array}{l}\text { LT } \\
\text { LT } \\
\text { LT } \\
\text { LT } \\
\text { LT } \\
\text { LT } \\
\text { LT } \\
\text { LT }\end{array}$ & $\begin{array}{r}-10 \\
25 \\
55 \\
120 \\
-30 \\
10 \\
90 \\
120\end{array}$ & $\begin{array}{r}14 \\
77 \\
131 \\
248 \\
-22 \\
50 \\
194 \\
248\end{array}$ & $\begin{array}{r}33.9 \\
77.2 \\
171.3 \\
189.8 \\
16.2 \\
139.7 \\
199.7 \\
215.0\end{array}$ & $\begin{array}{r}20.0 \\
45.6 \\
101.1 \\
112.0 \\
9.6 \\
82.4 \\
117.8 \\
126.9\end{array}$ & $\begin{array}{l}16.287 \\
14.92 \\
13.993 \\
13.27 \\
16.883 \\
15.389 \\
13.582 \\
12.655\end{array}$ & $\begin{array}{l}17.625 \\
18.201 \\
17.791 \\
16.18 \\
16.883 \\
18.23 \\
16.668 \\
15.584\end{array}$ \\
\hline
\end{tabular}


Table 10. (Contd.)

\begin{tabular}{|c|c|c|c|c|c|c|c|c|c|c|}
\hline \multirow{2}{*}{$\begin{array}{l}\text { Specimen } \\
\text { ID }\end{array}$} & \multirow[t]{2}{*}{ Location } & \multirow[t]{2}{*}{ Region ${ }^{a}$} & \multirow{2}{*}{$\begin{array}{c}\text { Fluenceb } \\
\left(\mathrm{n} / \mathrm{cm}^{2}\right)\end{array}$} & \multirow{2}{*}{$\begin{array}{l}\text { Orien- } \\
\text { tation }\end{array}$} & \multicolumn{2}{|c|}{ Test Temp. } & \multicolumn{2}{|c|}{ Impact Energy } & \multicolumn{2}{|c|}{ Load (kN) } \\
\hline & & & & & $\left({ }^{\circ} \mathrm{C}\right)$ & $\left({ }^{\circ} \mathrm{F}\right)$ & $\left(\mathrm{J} / \mathrm{cm}^{2}\right)$ & $(\mathrm{ft} \cdot 1 \mathrm{lb})$ & Yield & Maximum \\
\hline & & & & & \multicolumn{2}{|c|}{ Annealed } & & & & \\
\hline I!4R-15WA & 14 & 1 & $3 \times 10^{17}$ & LT & 0 & 32 & 69.6 & 41.1 & 16.15 & 19.256 \\
\hline I14R-17WA & 14 & I & & LT & 25 & $\pi 7$ & 130.7 & 77.1 & 14.696 & 18.406 \\
\hline 114R-19WA & 14 & I & & LT & 70 & 158 & 177.4 & 104.7 & 13.436 & 17.303 \\
\hline I14R-14WA & 14 & $\mathrm{o}$ & & LT & -40 & -40 & 51.5 & 30.4 & 15.106 & 19.09 \\
\hline 114R-16WA & 14 & o & & LT & 10 & 50 & 176.4 & 104.1 & 14.451 & 18.728 \\
\hline I14R-18WA & 14 & 0 & & LT & 40 & 104 & 188.3 & 111.1 & 13.895 & 18.23 \\
\hline 114R-20WA & 14 & o & & LT & 90 & 194 & 196.7 & 116.1 & 12.733 & 17.478 \\
\hline I15R-09WA & 15 & I & $3 \times 10^{17}$ & LT & -20 & -4 & 136.8 & 80.7 & 14.93 & 18.855 \\
\hline $115 R-11 W A$ & 15 & I & & LT & .5 & 59 & 156.1 & 92.1 & 13.69 & 18.025 \\
\hline I15R-13WA & 15 & I & & LT & 55 & 131 & 180.9 & 106.7 & 13.455 & 17.605 \\
\hline 115R-10WA & 15 & 0 & & LT & -10 & 14 & 123.7 & 73.0 & 15.34 & 19.021 \\
\hline I15R-12WA & 15 & 0 & & LT & 20 & 68 & 163.2 & 96.3 & 14.764 & 18.543 \\
\hline I15R-14WA & 15 & 0 & & LT & 120 & 248 & 195.8 & 115.5 & 13.036 & 16.824 \\
\hline
\end{tabular}

a $I$ and $O$ represent the inner- and outer- $10-\mathrm{mm}$ regions, respectively, across the wall. thickness.

b Represent the values at the inner surface of the wall. Fluence for the outer-region samples is estimated to be a factor of 1.5 lower than that for inner-region samples.

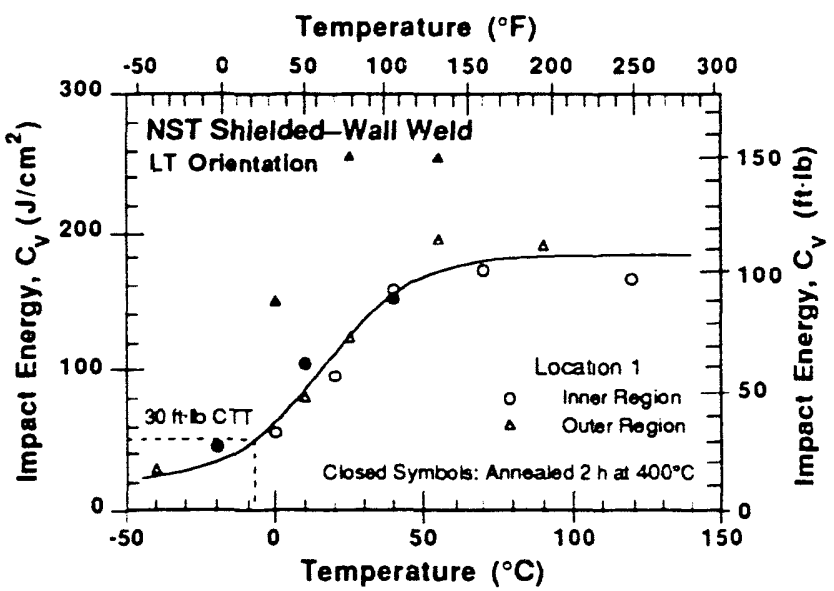

Figure 16.

Charpy transition curves for weld metal specimens from the inner- and outer-10$\mathrm{mm}$ regions of the shielded wall of the Shippingport NST

erties of nonirradiated weld metal. For example, annealing has little or no effect on weld specimens from the inner region of the shielded-wall weld, whereas the impact energy of weld specimens from the oriter region !ncreased significantly after annealing (Fig. 16). The increase in impact energy is most likely due to microstructural changes. The annealing data, therefore, cannot be used to characterize irradiation embrittlement of the weld syecimens.

Charpy transition curves of the weld metal specimens, with and without annealing for $2 \mathrm{~h}$ at $400^{\circ} \mathrm{C}$, are shown in Figs. 16 and 17. The best-fit curves to Eq. 1 are also shown in the figures: values of the constants in Eq. 1, as well as USE and CTT at the 20.4 J (15 ft.lb) and $41 \mathrm{~J}(30 \mathrm{ft} \cdot \mathrm{lb})$ level, are given in Table 11 . The impact strength of the shielded-wall weld, shown in Fig. 16, is significantly higher than that of the base metal. The $41-J$ (30 $\mathrm{ft} \cdot \mathrm{lb}) \mathrm{CTT}$ and USE of the weld are $7^{\circ} \mathrm{C}\left(28^{\circ} \mathrm{F}\right)$ and $184 \mathrm{~J} / \mathrm{cm}^{2}$ (109 ft.lb), respectively. Position through the thickness of the weld, i.e., inner and outer regions, has no effect on the transition curve. 
Table 11. Values of constants in Eq. 1. CTT, and USE for weld metal from the shielded and irradiated walls of the Shippingport NST

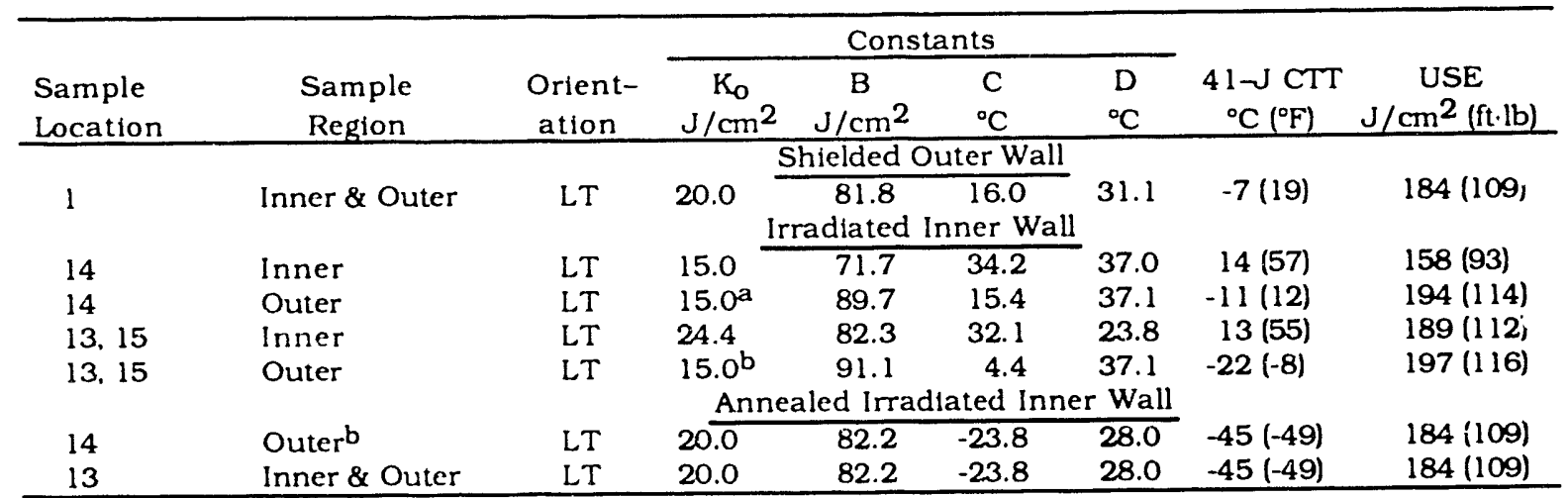

a Value of $\mathrm{K}_{\mathrm{O}}$ is assumed to be the same as that for location 14 inner region.

b Results for location 14 inner region agree well with those for the shielded outer wall.

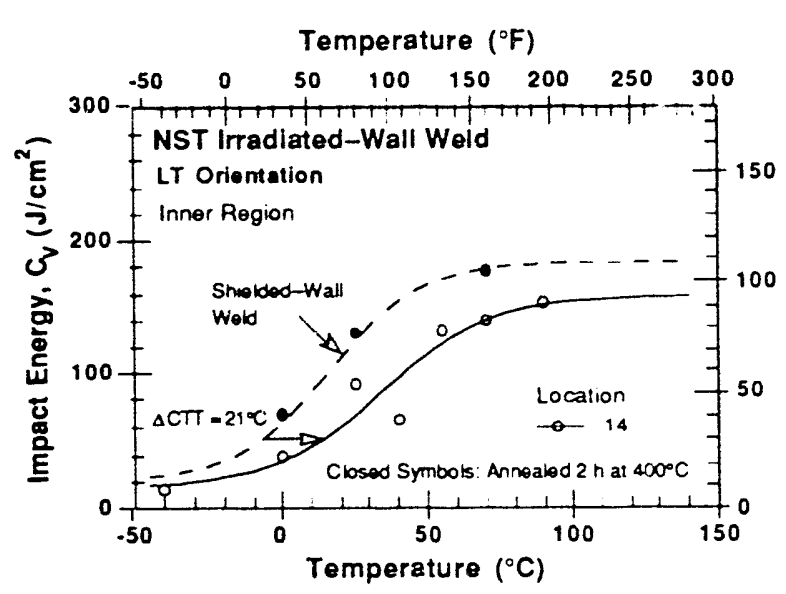

(a)

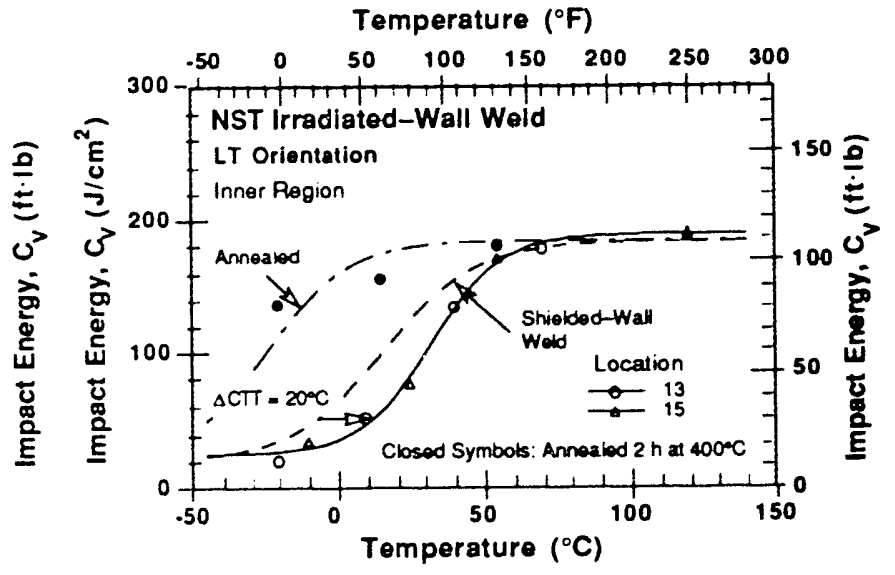

(b)

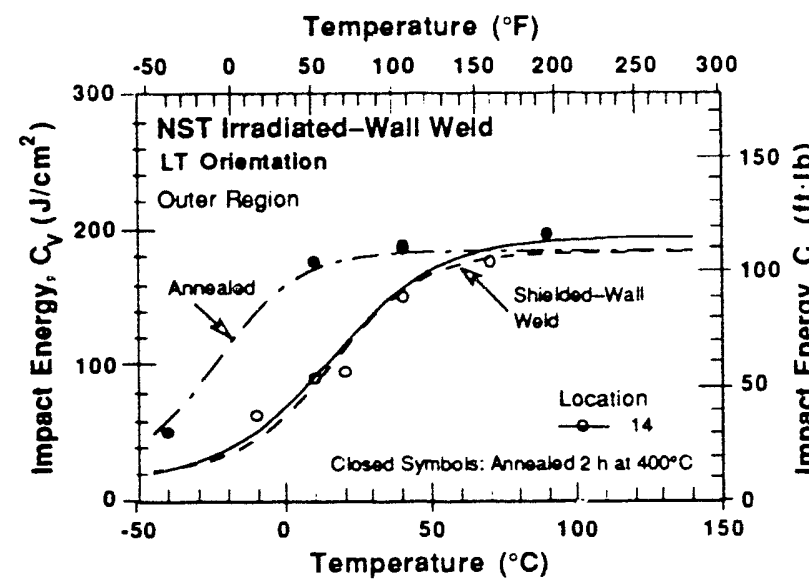

(c)

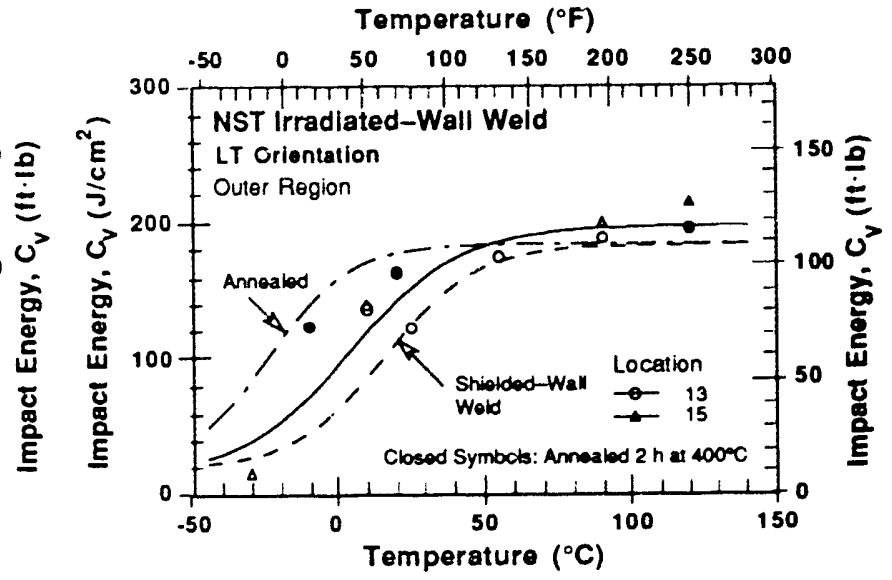

(d)

Figure 17. Charpy transition curves for weld metal specimens from (a) and (b) inner region and (c) and (d) outer region of the irradiated wall of the Shippingport NST 
Specimens from the irradiated-wall weld show a strong effect of location across the thickness of the wall. The transition curves for the inner region of the irradiated-wall welds are shown in Figs. 17a and 17b. The $41 \mathrm{~J}(30 \mathrm{ft} \cdot 1 \mathrm{~b})$ CTT is $14^{\circ} \mathrm{C}\left(57^{\circ} \mathrm{F}\right)$ for the weld at Location 14 and $13^{\circ} \mathrm{C}\left(55^{\circ} \mathrm{F}\right)$ for welds at Locations 13 and 15 , i.e., a shift of 21 and $20^{\circ} \mathrm{C}$ ( 38 and $36^{\circ} \mathrm{F}$ ), respectively, relative to the data for the shielded-wall weld. Weld specimens from the outer regions of the irradiated wall show no embrittlement relative to the shielded-wall weld. The Charpy transition curve of the outer-region specimens from Location 14 is comparable (Figs. 17c) and that for specimens from Locations 13 and 15 is slightly higher than that of the shielded-wall weld (Figs. 17d). Charpy data for annealed weld metal specimens from the irradiated wall seem to represent recovery from irradiation embrittlement as well as the effect of thermal aging caused by microstructural instabilities. The results for annealed specimens from the inner region of Location 14 alone indicate recovery from irradiation embrittlement, 1.e., the impact energies compare well with those for the shielded-wall weld. Specimens from inner and outer regions of Location 15 and the outer region of Location 14 show a large increase in impact energy after annealing.

\section{Discussion}

\subsection{HFIR Surveillance Data}

The Charpy-impact data for the Shippingport NST indicate that the shifi in CTT is not as severe as would be expected on the basis of the changes seen in HFIR surveillance samples. A summary of Charpy-impact and tensile data for HFIR surveillance samples, for samples irradiated in the ORR, and for the Shippingport NST are given in Table 12. In Fig. 18, Charpy transition curves for LT specimens from the shielded and irradiated walls of the Shippingport NST are compared with results for nonirradiated and irradiated HFIR surveillance samples of A212-B steel. The results for the nonirradiated materials, Fig. 18a, indicate that the impact strength of the HFIR material is significantly higher than that for the Shippingport NST; the $20.4 \sim \mathrm{J}(15 \mathrm{ft} \cdot \mathrm{lb}) \mathrm{CTT}$ for the HFIR material is $35^{\circ} \mathrm{C}\left(63^{\circ} \mathrm{F}\right)$ lower

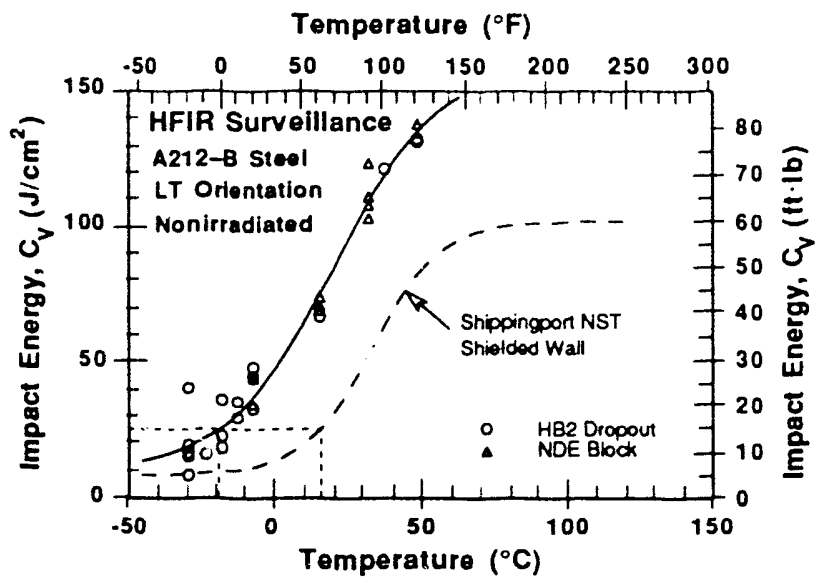

(a)

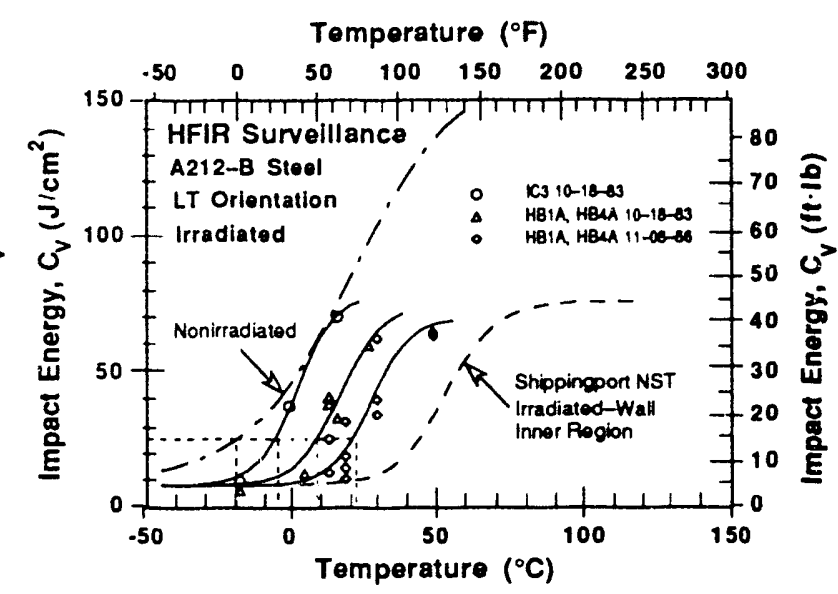

(b)

Figure 18. Comparison of Charpy transition curves for (a) nonirradiated and (b) irradiated HFIR surveillance samples and materlal from the Shippingport NST 
Table 12. Summary of Charpy-impact and tensile results for HFIR survellance tests, for samples irradiated in the ORR, and for the Shippingport NST

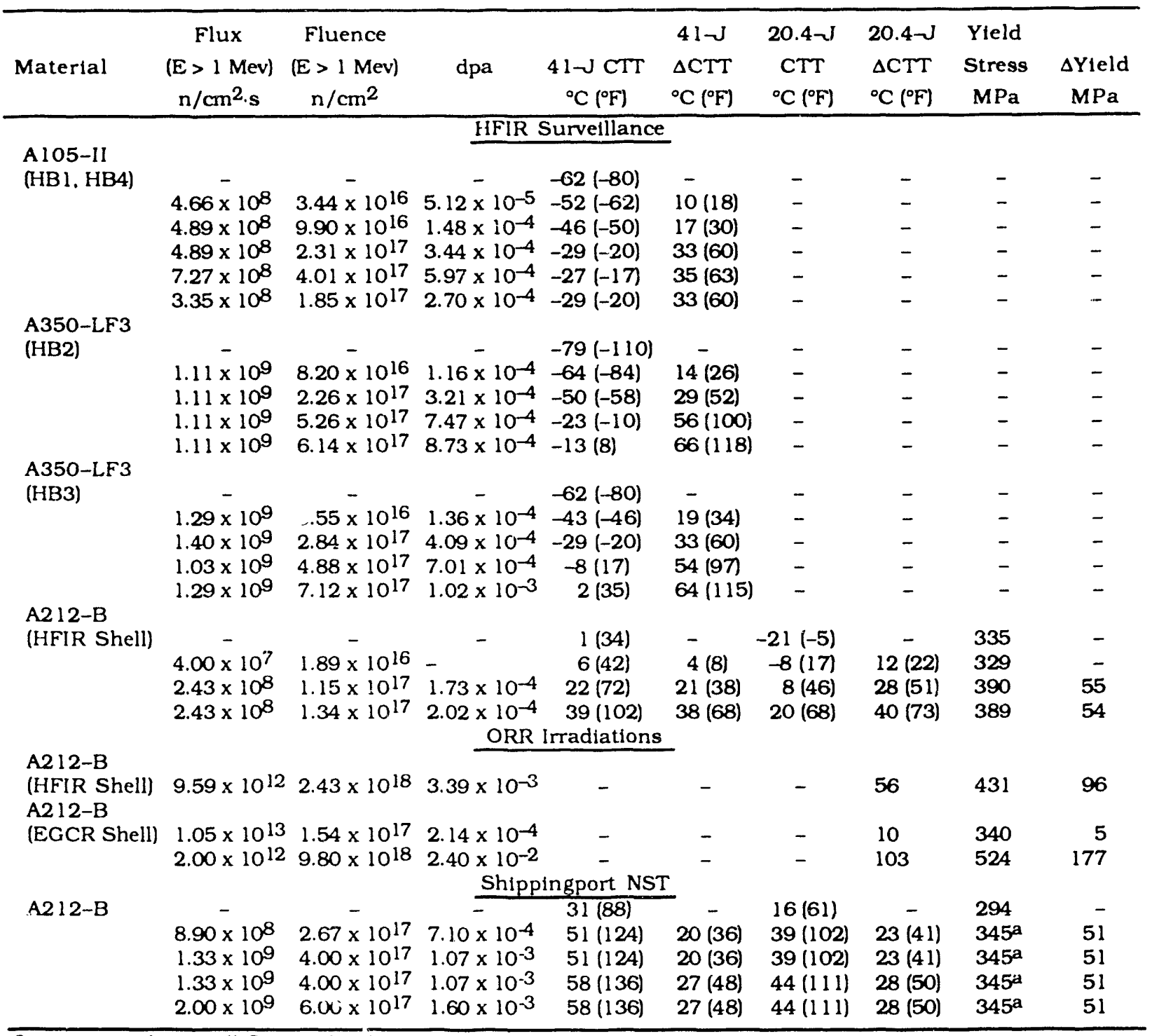

a Average value at all fluence levels.

and the USE is $\approx 60 \mathrm{~J} / \mathrm{cm}^{2}$ (35 ft.lb) higher than that for the Shippingport NST. After irradiation the shift in CTT is $40^{\circ} \mathrm{C}\left(73^{\circ} \mathrm{F}\right)$ for the HFIR material $\left(\approx 1.3 \times 10^{17} \mathrm{n} / \mathrm{cm}^{2}\right.$ fluence $)$ and $28^{\circ} \mathrm{C}\left(50^{\circ} \mathrm{F}\right)$ for the NST material $\left(=6 \times 10^{17} \mathrm{n} / \mathrm{cm}^{2}\right)$. Although the increase in CTr is smaller, the actual CTT of the Shippingport NST is significantly higher than that of the HFIR A2 12-B steel. At the service temperature of $55^{\circ} \mathrm{C}\left(131^{\circ} \mathrm{F}\right)$, the impact energy of the irradiated inner wall of the NST is very low, $\approx 40 \mathrm{~J} / \mathrm{cm}^{2}(\approx 24 \mathrm{ft} \cdot \mathrm{lb})$. Similar differences are also observed for the Charpy data on TL specimens.

The HFIR A2 12-B steel is not cnly tougher than the Shippingport NST steel, it is also stronger, 1.e., its tensile strength and hardness are higher than that of the NST material. The yield stress and hardness for nonirradiated materials are, respectively. $335 \mathrm{MPa}$ $(\approx 49 \mathrm{ksi})$ and $172 \mathrm{DPH}$ for the $\mathrm{HFIR}^{1}$ and $294 \mathrm{MPa}(43 \mathrm{ksi})$ and $156 \mathrm{DPH}$ for the 
Table 13. Chemical compositions (wt.\%) of ferritic steels from the HFIR surveillance program

\begin{tabular}{llccc}
\hline Element & $\begin{array}{c}\text { A212-B } \\
\text { Shell }\end{array}$ & $\begin{array}{c}\text { A105-II } \\
\text { HB1, HB4 }\end{array}$ & $\begin{array}{c}\text { A350-LF3 } \\
\text { HB2 }\end{array}$ & $\begin{array}{c}\text { A350-LF3 } \\
\text { HB3 }\end{array}$ \\
\hline C & 0.26 & 0.24 & 0.18 & 0.17 \\
Mn & 0.85 & 1.12 & 0.55 & 0.50 \\
P & 0.006 & 0.003 & 0.010 & 0.007 \\
S & 0.040 & 0.020 & 0.020 & 0.010 \\
S1 & 0.29 & 0.21 & 0.29 & 0.27 \\
Oa & 0.15 & 0.03 & 0.11 & 0.10 \\
N1 & $2.20^{a}$ & 0.14 & 3.30 & 3.20 \\
Cr & 0.075 & 0.042 & 0.090 & 0.080 \\
O & 0.0024 & 0.0033 & 0.0027 & 0.0026 \\
N & 0.0060 & 0.0063 & 0.0090 & 0.0083 \\
Ti & 0.010 & $<0.001$ & $<0.001$ & $<0.001$ \\
V & 0.0005 & 0.0005 & 0.0010 & $<0.001$ \\
Zr & $<0.001$ & $<0.001$ & $<0.001$ & $<0.001$ \\
Mo & 0.02 & 0.07 & 0.03 & 0.03 \\
A1 & 0.07 & 0.06 & 0.08 & 0.08 \\
Sn & 0.02 & $<0.005$ & 0.02 & 0.02 \\
B & $<0.005$ & $<0.005$ & $<0.005$ & $<0.005$ \\
\hline
\end{tabular}

a Believed to be high; another analysis showed 0.09

Shippingport NST. The chemical compositions of the ferritic steels from HFIR survellance program are given in Table 13. Except for the difference in copper content, elemental compositions of the A212-B steels from the HFIR pressure vessel and from the Shippingport NST are comparable. The differences in the transition curves of the nonirradiated A2 12-B steels are most likely due to microstructural factors, e.g., amount and distribution of inclusions.

The shifts in CTT of the material from the Shippingport NST, HFIR surveillance samples, and HFIR A2 12-B steel irradiated in the ORR are plotted as a function of neutron exposure in Fig. 19. The results for the Shippingport NST material are consistent with those from ORR-Irradiated steel. The HFIR survellance data follow a different trend; for a given fluence, the shifts in CTT are greater than those for the Shippingport NST material or the ORR-irradiated samples. The shifts in 41 $-J(30-\mathrm{ft} \cdot \mathrm{lb}) \mathrm{CTT}$ of A212-B steel from the HFIR surveillance program are not significantly different from those for A105-II or A350-LF3 steels.

\subsection{Low-Temperature Irradiation}

Irradiation temperature is an important factor in radiation damage, particularly in the temperature range of operation of power reactors. At temperatures $>232^{\circ} \mathrm{C}\left(>450^{\circ} \mathrm{F}\right)$, progressively lower embrittlement is observed with increasing temperature. ${ }^{13-15}$ However, relatively little or no temperature effect is observed at irradiation temperatures $<232^{\circ} \mathrm{C}$ $\left(<450^{\circ} \mathrm{F}\right)$. In Fig. 20 , the results for the Shippingport NST and for samples irradiated in the ORR are compared with data from test reactors $3,14-20$ and from Army reactors surveillance program 21.22 . The shifts in CTT for the NST A212-B and HFIR A212-B irradiated in the ORR are consistent with these results and represent the tall of the trend band that describes the increase in CTT of various steels irradiated at $<232^{\circ} \mathrm{C}$. An upper bound curve for low-temperature irradiation is expressed as 


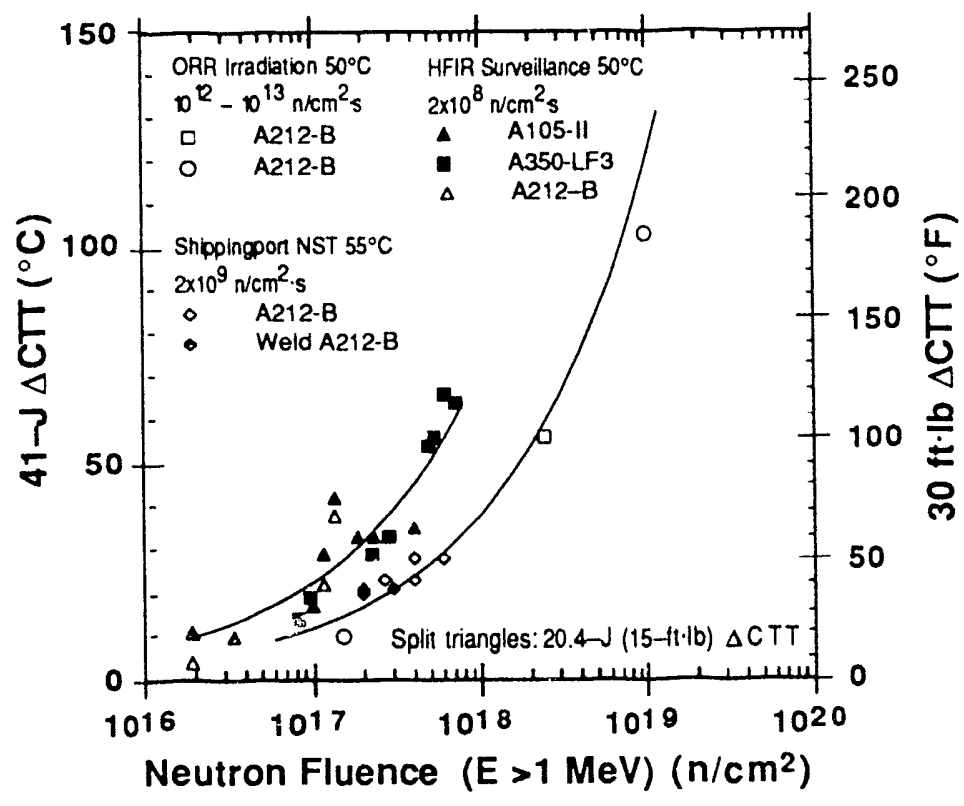

Figure 19.

Shifts in CTT with neutron fluence for the Shippingport NST material, HFIR surveillance samples, and HFIR A212-B irradiated in the ORR. The solid lines represent CTT shifts as a function of the square root of total fluence.

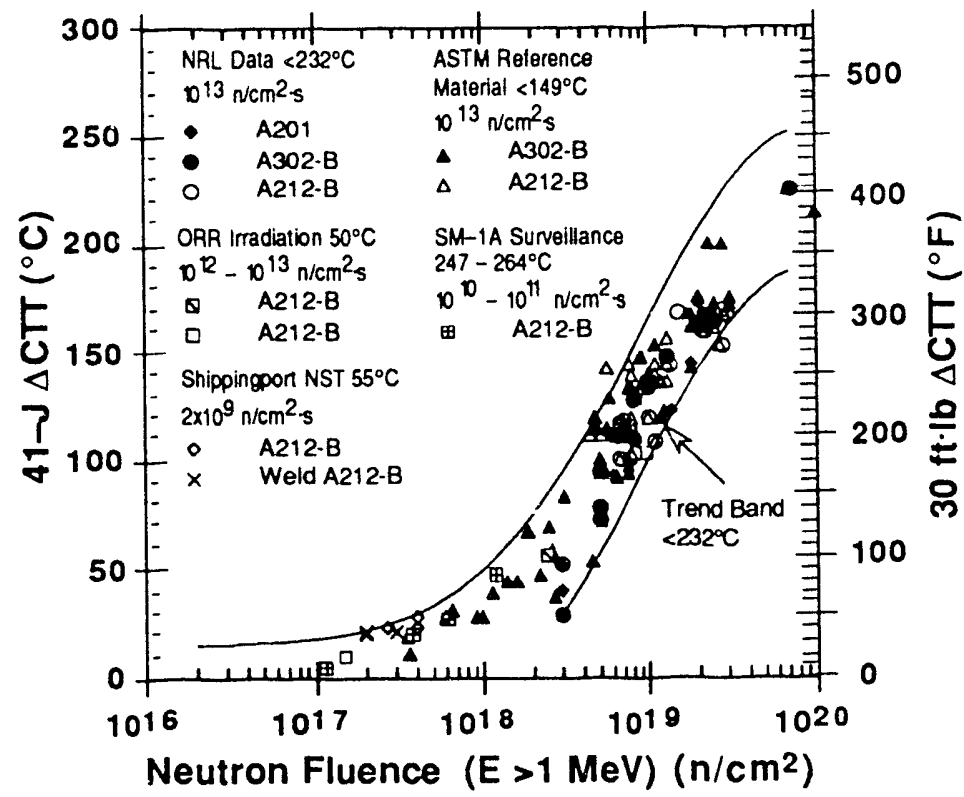

Flgure 20.

Comparison of CTT shifts for the Shippingport NST and for samples irradiated in the ORR with data from test reactors

$$
\Delta C T T=15+150 \text { (10.42 }+0.22 \log ),
$$

and the lower bound curve as

$$
\Delta \mathrm{CTT}=-50+150 \mathrm{f}(0.42+0.22 \log f)
$$

where the CTT shift is in ${ }^{\circ} \mathrm{C}$ and fluence $f$ is in $10^{19} \mathrm{n} / \mathrm{cm}^{2}$.

Swedish data ${ }^{23}$ on the effect of irradiation temperature on embrittlement of ferritic steels (including A212-B steel) indicate that embrittlement is relatively insensitive to temperature between 110 and $232^{\circ} \mathrm{C}\left(230\right.$ and $\left.450^{\circ} \mathrm{F}\right)$ and decreases at lower and higher temperatures. The data from irradiation of various steels and welds ${ }^{3,21}$ at $<.93^{\circ} \mathrm{C}\left(<200^{\circ} \mathrm{F}\right)$ fall 


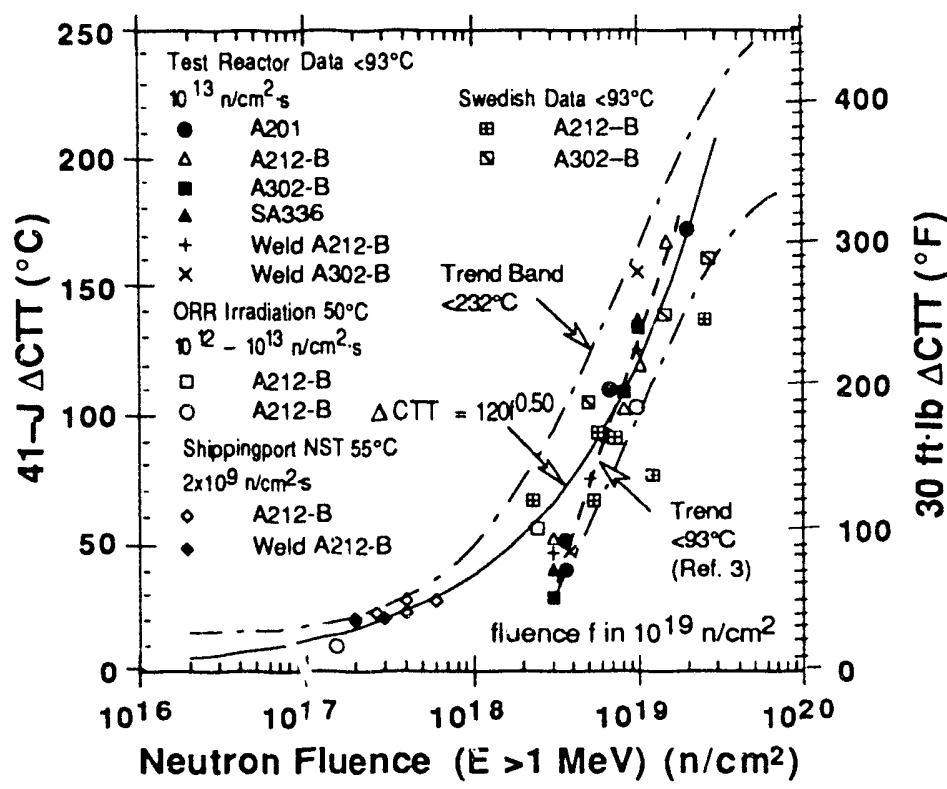

Figure 21.

Comparison of CTT shifts for the Shippingport NST and ORRirradiated specimens with test reactor data for irradiations at $<93^{\circ} \mathrm{C}$. The solid line is the bestfit curve.

between the center and lower-bound curve of the $<232^{\circ} \mathrm{C}$ trend band, Fig. 21 . Swedish data from irradiations i $110-230^{\circ} \mathrm{C}$ follow the upper bound of the trend band. Embrittlement of ferritic steels at $<93^{\circ} \mathrm{C}$ appear to be better represented by the average trend for low temperature irradiations. A power-law best fit of the data for various steels irradiated at $<232^{\circ} \mathrm{C}$ yields

$$
\Delta \mathrm{CTT}=120 \mathrm{O}^{0.50},
$$

where the CTT shift is in ${ }^{\circ} \mathrm{C}$ and fluence $\mathrm{f}$ is in $10^{19} \mathrm{n} / \mathrm{cm}^{2}$.

The data in Fig. 20 indicate that variations in chemical composition of steel, e.g., Cu or $\mathrm{Ni}$ contents, have little or no effect on irradiation embrittlement at temperatures $<232^{\circ} \mathrm{C}$. For example, the increases in CTT for ASTM reference A212-B and A302-B steels irradiated at $<149^{\circ} \mathrm{C}$ are comparable, Fig. 20. Compositional effects are observed for these steels when they are irradiated in the same facilities at higher temperatures. 20 In Fig. 22 , the shifts in CTT for the ASTM reference materials irradiated at $277-310^{\circ} \mathrm{C}\left(530-590^{\circ} \mathrm{F}\right)$ are compared with the $<232^{\circ} \mathrm{C}$ trend band. The results for $\mathrm{A} 302-\mathrm{B}$ steel from the YankeeRowe surveillance program are also included in the Fig. 22.20 For a given fluence level, the increase in CTT for A2 12-B steel (with 0.26 wt.\% Cu and 0.28 wt.\% $\mathrm{Ni}$ ) is greater than that for A302-B steel (with 0.20 wt.\% $\mathrm{Cu}$ and 0.18 wt.\% Ni).

Surveillance data for Army reactors indicate no significant difference in embrittlement between A2 12-B and A350 steels. 16.21,22 The results for A350 steels in Army reactors, shown in Fig. 23, are within the trend band for irradiations at $<232^{\circ} \mathrm{C}$, although they are close to the upper-bound curve. HFIR survelllance data for A350-LF3 and A105-II steels do not follow the trend for A350 Army reactor steels; they show greater embrittlement than the $<232^{\circ} \mathrm{C}$ trend band. These results indicate that the greater embrittlement of HFIR surveillance samples relative to the Shippingport NST is primarily due to factors other than material and compositional differences. 

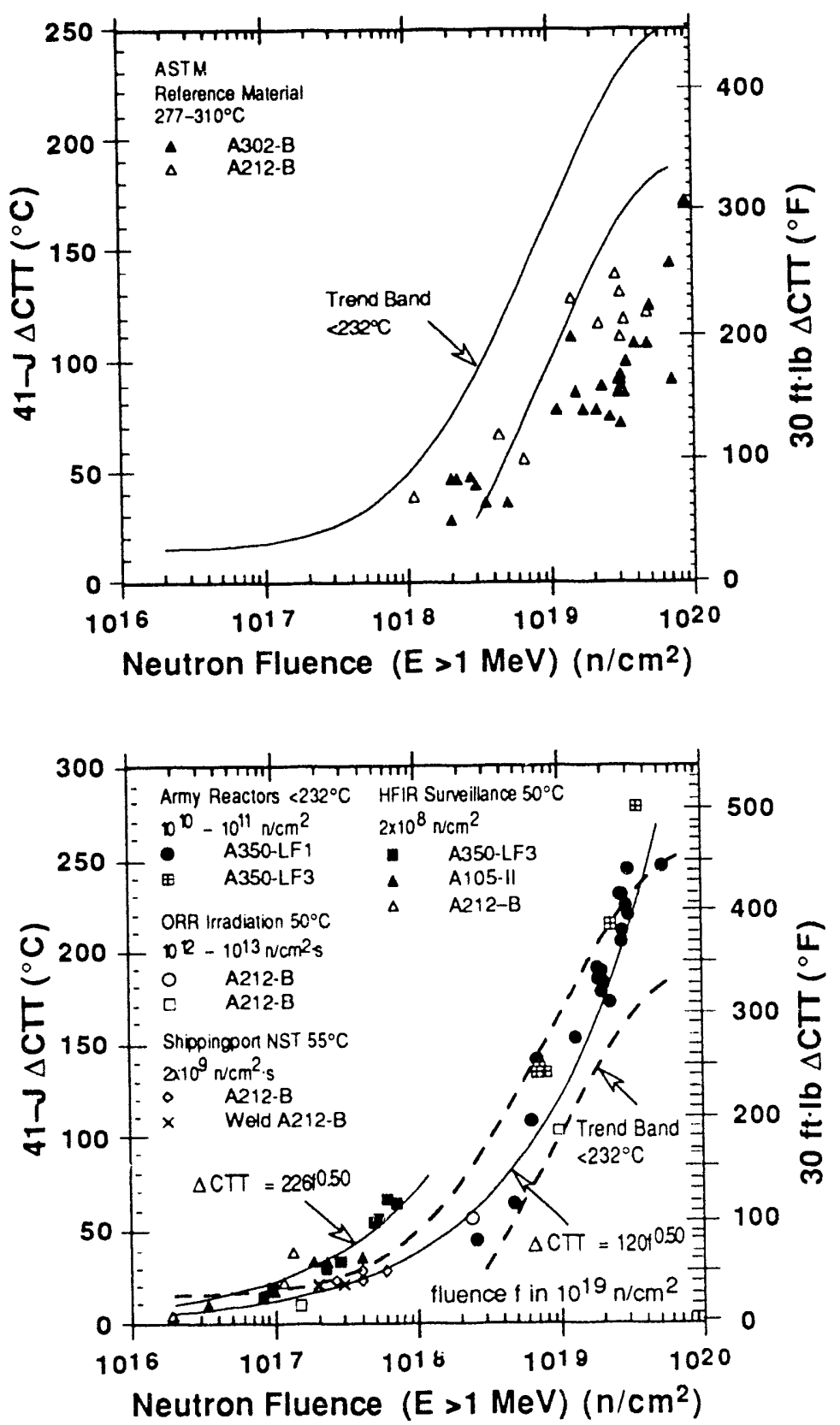

Figure 22.

Shifts in CTT with neutron fluence for ASTM reference A212-B and $A 302-B$ steels irradiated at 277$310^{\circ} \mathrm{C}$
Figure 23.

Comparison of CTT shifts for HIFIR surveillance samples, the Shippingport NST, and ORRirradiated samples with data from test and Army reactors

To account for differences in the spectra of the various irradiation facilities, the increase in CTT for HFIR surveillance steels, ORR-irradiated samples, and the Shippingport NST, as well as test reactor data at $<93^{\circ} \mathrm{C}$, are plotted in Fig. 24 as a function of displacement per atom (dpa) for $\mathrm{E}>0.1 \mathrm{MeV}$ rather than fluence. The difference still exists between HFIR surveillance data and results from ORR irradiation, the Shippingport NST, or test reactors.

\footnotetext{
* From Ref. 4 , where the test reactor trend curve was obtained assuming that the calculated spectrum for ORR was appropriate for test reactor data.
} 


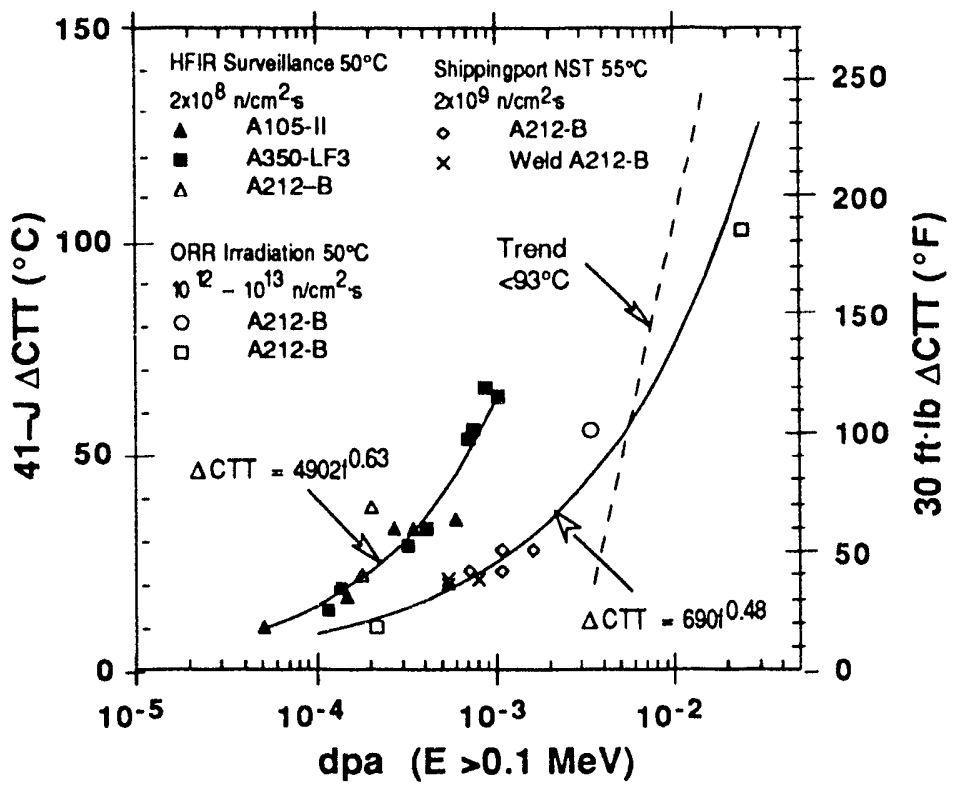

Figure 24.

Comparison of shifts in CTT with dpa for HFIR survellance samples, the Shippingport NST. and ORR irradiation with data from test reactor

\subsection{Effect of Fluence Rate}

Radiation embrittlement depends on the fraction of point defects that avoid recombination and survive to form clusters, rather than on point-defect production, which is conventionally represented by fast neutron fluence or by dpa. Radiation effects under different irradiation conditions will correlate with fast fluence or dpa if the relative fraction of point defects that avoid recombination remains the same for different irradiation conditions. However, the overall rate of radiation hardening and embrittlement will be accelerated by any mechanism that decreases the rate of point-defect recombination relative to their production rate. Two such mechanism, based on bulk recombination or rate effect and on incascade recombination or spectral effects, have been considered to explain the greater embrittlement of HFIR surveillance samples. ${ }^{24}$ Lower displacement rates decrease the fraction of bulk recombination, thereby a larger fraction of point defects survive for formation of clusters. The rate effect may contribute to accelerated embrittlement of HFIR samples because the flience rate is nearly 5 orders of magnitude lower for HFIR than for the test reactors. The spectral effect considers that, for irradiations producing small cascades, e.g., recoils from thermal neutron capture, a smaller fraction of point defects recombine in the cascade and the available number of defects per displacement is larger than for irradiations that produce large cascades. Thus, the contribution to radiation embrittlement from thermal neutrons is larger per dpa than that from fast neutrons.

The greater embrittlement of HFIR survellance samples relative to that of specimens irradiated in the ORR and test reactors has been evaluated on the basis of a fluence-rate effect. ${ }^{4}$ The rate effect was established from specimens irradiated at a fluence rate of $\leq 1 \times 10^{10} \mathrm{n} / \mathrm{cm}^{2} \cdot \mathrm{s}$; recent data indicate that at $\approx 93^{\circ} \mathrm{C}\left(\approx 200^{\circ} \mathrm{F}\right)$ there is essentally no rate effect in the fast flux range of $1 \times 10^{10}-3 \times 10^{13} \mathrm{n} / \mathrm{cm}^{2} \cdot \mathrm{s} .25$ At temperatures $<93^{\circ} \mathrm{C}\left(200^{\circ} \mathrm{F}\right)$, vacancies are relatively immobile, which reduces the importance of rate effect.24 HFIR survelllance data for the A350-LF3 and A212-B steels, corresponding to flux values of $2.4 \mathrm{x}$ $10^{8}$ and $1.2 \times 10^{9} \mathrm{n} / \mathrm{cm}^{2} \cdot \mathrm{s}$, respectively, were used to obtain plots of dpa vs. dpa rate for 


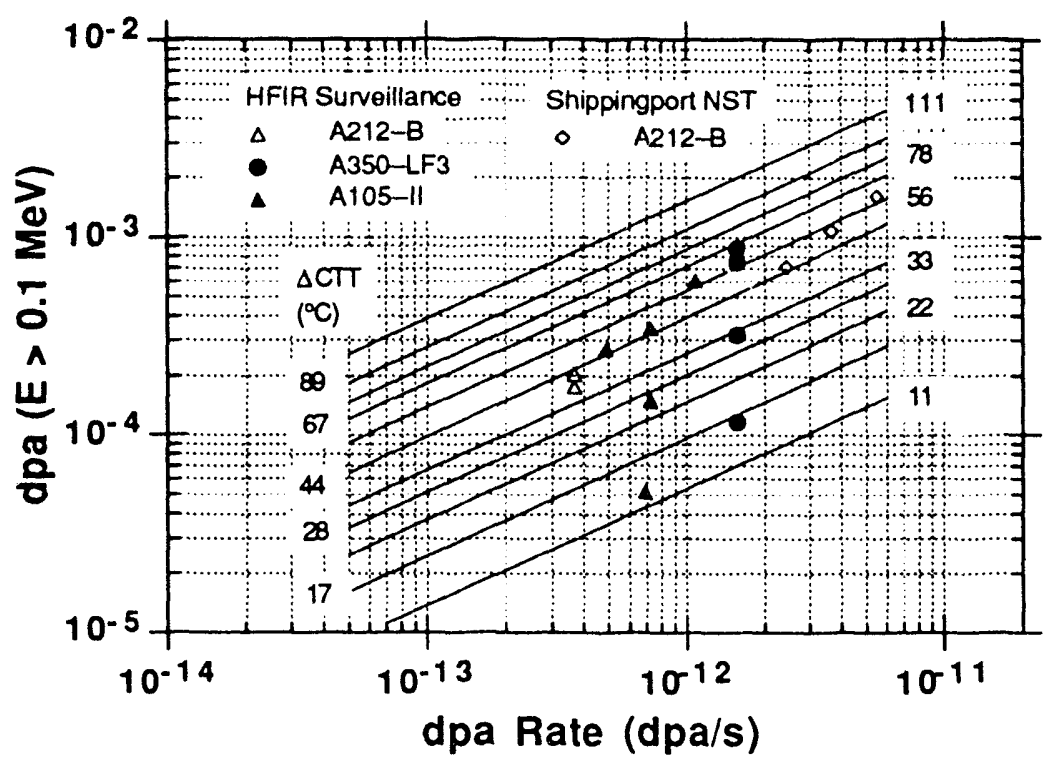

Figure 25.

Plots of dpa (E >0.1 MeV) vs. dpa rate for spectic values of CTT shifts (Ref. 4)

specific CTT shifts in Fig. 25. The irradiation conditions (1.e., dpa vs. dpa/s) for the Shippingport NST steel are also shown in Fig. 25. The fluence rate for the Shippingport NST is comparable to thie rates for HFIR A350-LF3 or A105-II steels, i.e., $\approx 1 \times 10^{9} \mathrm{n} / \mathrm{cm}^{2} \cdot \mathrm{s}$. If fluence rate has a large effect on the CTT shifts, the values for the Shippingport NST should be close to those for A350-LF3 and A105-Il steels. The predicted increase in CTT of $45-60^{\circ} \mathrm{C}\left(81-108^{\circ} \mathrm{F}\right)$ from Fig. 25 for the Shippingport NST, is higher by a factor of $\approx 2$ than the measured shifts in CTT. The predicted CTT shifts for A105-II steel also show poor agreement with the experimental data (Table 12). The results indicate that fluence rate has no effect on the shift in CTT at values as low as $2 \times 10^{9} \mathrm{n} / \mathrm{cm}^{2} \cdot \mathrm{s}$. The greater embrittlement of HFIR surveillance samples is most likely due to spectral effects.

\subsection{Spectral Effects}

The HFIR spectrum is highly thermalized, with thermal neutrons accounting for $96 \%$ of the total flux in the surveillance position. In the Shippingport and most test reactors of interest, the thermal neutron flux is a much smaller fraction of the total flux. Thermal neutrons cannot cause damage by elastic scattering since the minimum neutron energy required to displace an atom is above $0.5 \mathrm{keV}$. Damage associated with thermal neutrons is caused by recoil events resulting from neutron capture, 1.e., from the $(n, \gamma)$ reaction and for some cases the $(n, \alpha)$ reaction. As was discussed in Section 4.3, radiation effects depend on the fraction of point defects that survive rather than on the production of defects. Slow neutrons in an energy range $<0.1 \mathrm{MeV}$ increase the avallable point defects per unit displacement, resulting in enhanced cluster formation and embrittlement. Contributions of slow neutrons are not accounted for in conventional methods for assessing damage in terms of fast-neutron fluence $(E>1 \mathrm{MeV})$ or dpa $(E>0.1 \mathrm{MeV})$.

Alternative damage models have been used to determine the fraction of stable point defects produced by a given irradiation. Calculations based on damage efficiency, i.e., the probability of forming a stable defect, were recently completed to explain the discrepancy 
between the shifts in CTT of HFIR survellance and ORR-irradiated samples, and the Shippingport NST. The models proposed by Doran ${ }^{26}$ and Wiedersich 27 were used for damage efficiency. The procedure for calculating dpa was modified to include damage efficiency, and the relative changes in damage rate, rather than absolute damage rates, were determined for the neutron flux spectra for Shippingport, HFIR, and ORR.

The results indicate that the contributions from thermal neutrons for HFIR, ORR, and Shippingport samples are, respectively, 29, 2.5, and $0.4 \%$ of the total damage. Relative changes in damage rate from the Wiedersich model appear to be of the right order to explain the observed differences in embrittlement between samples from these three facilities. The ratio of modified damage rates is 3.3-5.7 for HFIR/Shippingport, 4.4 for HFIR/ORR, and 0.8-1.3 for ORR/Shippingport. The relative changes from the Doran model, e.g., 1.2-1.6, are not sufficient to explain the discrepancies.

A similar approach has also been used to show that spectral effects may dominate the accelerated embrittlement of HFIR surveillance samples. 24 The shifts in CTT for HFIR surveillance and ORR-irradiated samples fall along a single curve when plotted as a function of thermal neutron fluence $(\mathrm{E}<0.4 \mathrm{eV})$. Similar correspondence is observed for the increases in tensile stress for HFIR surveillance and out-of-core ORR-irradiated samples ${ }^{2}$ plotted as a function of thermal neutron fluence.

Other analytical models of freely migrating interstitials and vacancies also show the importance of spectral effects and are consistent with the differences in property data from different facilities. 28 The best correlation of the data from HFIR surrellance and irradiations in the ORR, Omega West reactor, and the Rotating Target Neutron Source is obtained when increases in yield stress are compared on the basis of freely migrating interstitial defects, which are believed to better represent the defects participating in radiation hardening.

These results indicate that in neutron environments with high thermal-to-fast ratio and low temperatures, current measures of radiation damage, such as fast fluence and dpa, are inadequate. Alternative measures of damage assessment must be considered for cases of a softened neutron spectrum such as HFIR.

\section{Conclusions}

Characterization of material from the Shippingport NST indicates that the embrittlement of this A2 12 Grade B steel in a low-temperature, low-flux environment is consistent with the trend band for irradiations at $<232^{\circ} \mathrm{C}\left(<450^{\circ} \mathrm{F}\right)$ and shows good agreement with data from test and Army reactors. The shifts in CTT are between 23 and $28^{\circ} \mathrm{C}(41$ and $50^{\circ} \mathrm{F}$ ). The NST weld metal is significantly tougher than the plate material; shift in CTT is $=20^{\circ} \mathrm{C}\left(36^{\circ} \mathrm{F}\right)$. These shifts are significantly lower than those expected on the basis of results obtained from the HFIR surveillance samples. The results indicate that fluence rate does not affect radiation embrittlement at rates as low as $2 \times 10^{8} \mathrm{n} / \mathrm{cm}^{2} . \mathrm{s}$ and the low operating temperatures of the Shippingport NST, 1.e., $55^{\circ} \mathrm{C}\left(130^{\circ} \mathrm{F}\right)$. The accelerated embrit-

\footnotetext{
"Greenwood, L., "Damage Calculations for Shippingport, HFIR, and ORR," memo to W. J. Shack, October 1989.
} 
tlement of HFIR survellance samples are most likely due to the contribution of thermal neutrons.

\section{Acknowledgments}

This work was supported by the Office of Nuclear Regulatory Research, U. S. Nuclear Regulatory Commission and the Office of LWR Safety and Technology, U.S. Department of Energy. The authors are grateful to W. F. Burke and G. M. Dragel for their contributions to the experimental effort and to A. Sather for conducting the mechanical tests.

\section{References}

1. R. K. Nanstad, K. Farrell, D. N. Braski, and W. R. Corwin, "Accelerated Neutron Embrittlement of Ferritic Steels at Low Fluence: Flux and Spectrum Effects," J. Nucl. Mater., 158, 1-6 (1988).

2. R. D. Cheverton, I. G. Merkle, and R. K. Nanstad, Evaluation of HFIR Pressure Vessel Integrity Considering Radiation Embrittlement, ORNL/TM-10444, Oak Ridge National Laboratory. Oak Ridge, TN (April 1988).

3. J. R. Hawthorne. "Studies of Radiation Effects and Recovery of Notch Ductility of Pressure Vessel Steels," in Symp, on Steels for Reactor Pressure Círcuits, British Nuclear Energy Conference, Iron and Steel Institute. London, 343-369 (1961).

4. R. D. Cheverton, F. B. Kam, R. K. Nanstad, and G. C. Robinson, "An Embrittlement Rate Effect Deduced from HFIR that may Impact LWR Vessel Support Life Expectancy." Nucl. Eng. and Des., 117. 349-355 (1989).

5. S. T. Rosinski, O. K. Chopra, and W. J. Shack, "Shippingport Neutron Shield Tank Sampling and Analysis Program," in Proc. 1989 ASME Pressure Vessel and Piping Conference, D. L. Marriott, T. R. Mager, and W. H. Bamford, eds., Vol. 170, ASME, New York, pp 109-113 (1989).

6. S. T. Rosinski, W. J. Shack, and O. K. Chopra, "Irradiation Embrittlement Investigation of the Shippingport Station Neutron Shield Tank," in Proc. Fourth Int. Symp. on Environmental Degradation of Materials in Nuclear Power Systems -- Water Reactors, D. Cubicciotti, ed., NACE. Houston. TX. pp 2.79-2.89 (1990).

7. O. K. Chopra. S. T. Rusinski, and W. J. Shack, "Embrittlement of the Shippingport Reactor Neutron Shield Tank," in Fatigue, Degradation, and Fracture - 1990, W. H. Bamford, C. Becht, S. Bhandari, J. D. Gilman, L. A. James, and M. Prager, eds., MPC Vol. 30, PVP Vol. 195, ASME, New York, pp. 157-168 (1990).

8. W. L. Server, "Impact Three-Point Bend Testing for Notched and Precracked Specimens," J. Test. and Eval., 6, 29 (1978). 
9. G. R. Odette, and G. E. Lucas, Irrad'atton Embrittlement of LWR Pressure Vessel Steels, EPRI Report NP-6114, Electric Power Research Institute, Palo Alto, CA (January 1989).

10. G. R. Odette, and G. E. Lucas, "Irradiation Embrittlement of Reactor Pressure Vessel Steels: Mechanisms, Models and Data Correlation," ASTM STP-909, American Society for Testing and Materials. Philadelphia, 206 (1986).

11. G. Sines and R. Carlson, "Hardness Measurements for Determination of Residual Stresses," ASTM Bulletin. 180, 35-37 (TP31-TP33) (1952).

12. A. Dervishyan, "The Stress-Hardness Relation," ASTM Bulletin, 215, 71-75 (TP127TP131) (1956).

13. R. W. Nichols and D. R. Harries, "Brittle Fracture and Irradiation Effects in Ferritic Pressure Vessel Steels," in Radiation Effects on Metals and Neutron Dosimetry. ASTM STP 341, American Society for Testing and Materials, Philadelphia, 162-198 (1963).

14. G. D. Whitman, G. C. Robinson, Jr., and A. W. Savolainen, Technology of Steel Pressure Vessels for Water-Cooled Nuclear Reactors, ORNL-NSIC-21, Oak Ridge National Laboratory, Oak Ridge. TN. (December 1967).

15. L. E. Steele and J. R. Hawthorne, "New Information on Neutron Embrittlement and Embrittlement Relief of Reactor Pressure Vessel Steels," in Flow and Fracture of Metals in Nuclear Envtronment. ASTM STP 380, American Society for Testing and Materials, Philadelphia, 283-311 (1965).

16. L. E. Steele and J. R. Hawthorne, "Neutron Embrittlement of Reactor Pressure Vessel Steels," in Materials and Fuels for High Temperature Nuclear Energy Applications, The MIT Press, Cambridge, MA., 366-409 (1964).

17. L. E. Steele and C. Z. Serpan. Jr., "Neutron Embrittlement of Pressure Vessel Steels," in Analysis of Reactor Vessel Radiation Effects Surveillance Programs. ASTM STP 481. American Society for Testing and Materials, Philadelphia, 49-101 (1966).

18. C. F. Carpenter, N. R. Knopf, and E. S. Byron, "Anomalous Embrittling Effects Observed During Irradiation Studies on Pressure Vessel Steels," Nucl. Sc. and Eng., 19, 18-38 (1964).

19. J. R. Hawthorne and L. E. Steele, "Metallurgical Variables as Possible Factors Controlling Irradiation Response of Structural Steels," in Effects of Radiation on Structural Metals, ASTM STP 426, American Society for Testing and Materials, Philadelphia, 534-572 (1967).

20. J. R. Hawthorne, Radiation Effects Information Generated on the ASTM Reference Correlation-Monitor Steel.s, i.STM Data Series Publication DS-54, American Society for Testing and Materials, Philadelphia. PA (1974). 
21. L. E. Steele, J. R. Hawthorne. C. Z. Serpan, Jr., E. P. Klier, and H. E. Watson, Irradiation Materials Evaluation and Reactor Pressure Vessel Surveillance for the Army Nuclear Power Program. U.S. Naval Research Laboratory Memorandum Report 1644 (September 1965).

22. L. E. Steele and C. Z. Serpan, Jr., "Army Reactor Vessel Survelllance and Vessel Examination," in Analysis of Reactor Vessel Radiation Effects Surveillance Programs, ASTM STP 481. American Society for Testing and Materials, Philadelphia, 105-135 (1966).

23. M. Grounes, "Review of Swedish Work on Irradiation Effects in Pressure Vessel Steels and on Significance of Data Obtained," in Effects of Radiation on Structural Metals, ASTM STP 426, American Society for Testing and Materials, Philadelphia, 224-259 (1967).

24. L. K. Mansur and K. Farrell, "On Mechanisms by which a Soft Neutron Spectrum may Induce Accelerated Embrittlement," J. Nucl. Mater., 170, 236-245 (1990).

25. M. L. Hamilton and H. L. Heinesch, "Tensile Properties of Neutron Irradiated A212B Pressure Vessel Steel," in 14th Int. Symp. on Effects of Radiation on Materials, ASTM STP 1046. American Society for Testing and Materials, Philadelph!a, 45-54 (1990).

26. D. G. Doran, R. L. Simons, and W. N. McElroy, "Spectral Effects in Neutron and Charged Particle Irradiadions." in Properties of Reactor Structural Alloys after Neutron or Particle Irradiation. ASTM STP 570. American Society for Testing and Materials, Philadelphia, 290-310 (1975).

27. H. Wiedersich, "Effects of the Primary Recoil Spectrum on Long Range Migration of Defects." Radiat. Eff., 113 (1-3). 97-107 (1990)

28. H. L. Heinisch, "Correlation of Mechanical Property Changes in Neutron-Irradiated Pressure Vessel Steels on the Basis of Spectral Effects," Fusion Reactor Materials, Semiannual Progress Report, March 31, 1989, DOE-ER-0313/6, 51-56 (1989). 
Internal:

O. K. Chopra (20)

H. M. Chung

T. F. Kassner

C. Malefyt (2)
S. T. Rosinski, SNL (25)

W. J. Shack (5)

C. E. Till

R. W. Weeks
TIS Files (3)

ANL Patent File

AivL Contract File

\section{External:}

NRC, for distribution per R5

ANL Libraries (2)

SNL Libraries (5)

Manager, Chicago Operations Office, DOE

Materials and Components Technology Division Review Committee

$H$. Berger, Industrial Quality, Inc., Gaithersburg, MD

M. S. Dresselhaus, Massachusetts Institute of Technology, Cambridge, MA

S. J. Green, Electric Power Research Institute, Palo Alto, CA

R. A. Greenkorn. Purdue U., West Lafayette, IN

C.-Y. Li, Cornell U., Ithaca, NY

P. G. Shewmon, Ohio State U., Columbus

R. E. Smith, Electric Power Research Institute, NDE Ctr., Charlotte, NC

D. Atteridge, Battelle Pacific Northwest Laboratury

W. H. Bamford, Westinghouse Electric Corp., Pittsburgh

R. D. Cheverton, Oak Ridge National Laboratory, Oak Ridge. TN

A. Cowan, Risley Nuclear Power Development Labs., Risley. Warrington, UK

W. H. Cullen. Materials Engineering Associates, Inc., Lanhem, MD

B. J. L. Darlaston, Berkeley Nuclear Laboratories, Be-keley jloucest rshire, UK

H. Farrar, Rockwell International, Rocketdyne Divisic.. Canoga Park. CA

J. Gilman, Electric Power Research Inst., Palo Alto, CA

L. Greenwood, Battelle Pacific Northwest Laboratory

M. Guttmann, Electricité de France, Les Renardieres Roule de Sens, France

D. L. Harrison, Office of LWR Safety and Technology, U.S. Dept. of Energy, Washington, DC:

J. R. Hawthorne, Materials Engineering Associates, Lariham, MD

P. Hedgecock, NUTECH Engineers, San Jose, CA

B. Hemsworth, HM Nuclear Installations Inspectorate, London

C. G. Interrante, Center for Materials Science, National Institute of Standards and Technology, Gaithersburg, MD

C. E. Jaske, CC Technologies, Cortest, Columbus, $\mathrm{OH}$

P. M. Lang, Office of Converter Reactor Deployment, U.S. Dept. of Energy, Washington, DC

M. Lapides, Electric Power Research Institute. Palo Alto, $(i$.

G. J. Licina. Structural Integrity Associates, San Jose, CA.

T. R. Mager, Westinghouse Electric Corp.. Pittsburgh

Y. Meyzaud. Framatome, Paris

R. K. Nanstad, Oak Ridge National Laboratory, Oak Ridge, TN

M. Prager. Materials Properties Council. Inc.. New York 
P. H. Pumphrey, National Power, Technology and Environment Center, Leatherhead. Surrey, UK

V. N. Shah, EG\&G Idaho, Inc., P. O. Box 1625, Idaho Falls, ID

G. Slama, Framatome, Paris La Defense, France

G. D. W. Smith, Oxford University, Oxford, UK

R. E. Stoller, Oak Ridge National Laboratory, Oak Ridge. TN

L. Taylor, Nuclear Electric plc., Chelsford Rd., Knutsford, Cheshire, UK

J. M. Vitek, Oak Ridge National Laboratory 

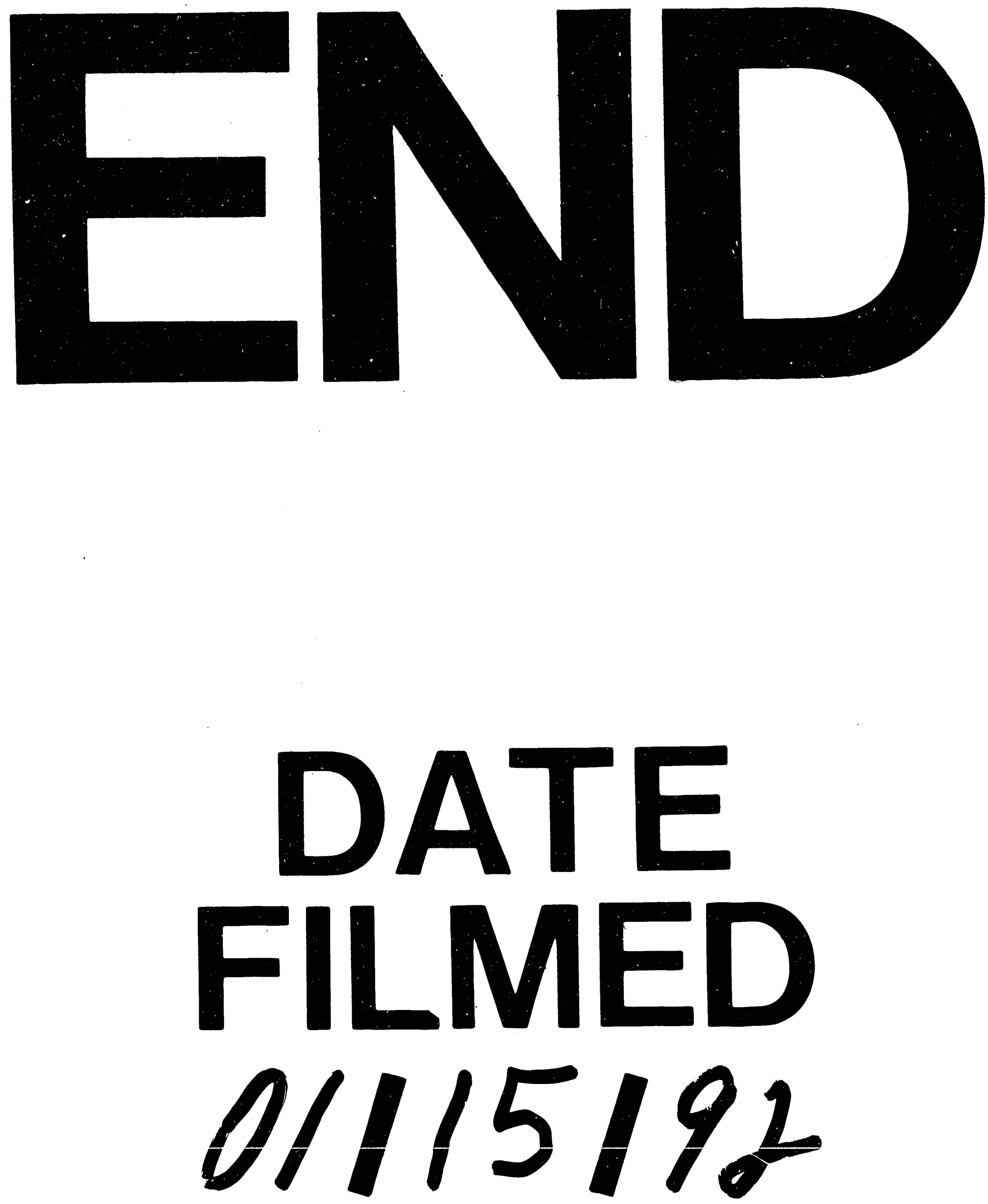
